On the inverse scattering problem in the acoustic environment

Ran Duan and Vladimir Rokhlin

Technical Report YALEU/DCS/TR-1395

March 3, 2008 
In this report, we construct numerical algorithms for the solution of inverse scattering problems in layered acoustic media. Our inverse scattering schemes are based on a collection of so-called trace formulae, and can be viewed as extension of the work started in [3].

The speed $c$ of propagation of sound, the density $\rho$, and the attenuation $\gamma$ are the three parameters reconstructed by the algorithm, given that all of them $(\rho(x, y, z), c(x, y, z), \gamma(x, y, z))$ are laterally invariant, i.e., depend only on the coordinate $z$. For a medium whose parameters $c, \rho$, and $\gamma$ have $m \geq 1$ continuous derivatives, and data measured for all frequencies $\omega$ on the interval $[-a, a]$, the error of our scheme decays as $1 / a^{m-1}$ as $a \rightarrow \infty$. In this respect, our algorithm is similar to the Fourier Transform. Our results are illustrated with several numerical examples.

\title{
On the inverse scattering problem in the acoustic environment
}

\author{
Ran Duan and Vladimir Rokhlin \\ Technical Report YALEU/DCS/TR-1395
}

March 3, 2008

The authors were supported in part by the Schlumberger Technology Corporation, ONR Grant N00014-07-1-0711, and AFOSR Grant FA9550-06-1-0239.

Approved for public release: Distribution is unlimited.

Keywords: inverse, scattering, Helmholtz, impedance, trace, acoustic 


\section{Introduction}

Inverse scattering has been an active field of research in science, mathematics, and engineering over the past several decades (see e.g. [2], [3], [5], [7], [9], [10], [11], [13]). It has applications in a wide range of fields, such as radar, medical imaging, oil exploration, microscopy, etc. However, constructing reliable and efficient algorithms for the solution of inverse scattering problems involves three major difficulties. First, inverse scattering problems are highly nonlinear except for the one-dimensional case, where the nonlinear problem can be reduced to a linear one, although the procedure of the reduction is numerically unstable. Second, numerical stability tends to be a problem, except in one dimension. Third, the time and memory requirements are beyond the capabilities of present computers via currently used methods. In this paper, we construct numerical algorithms for the solution of inverse scattering problems in the acoustic environment in three dimensions. Our inverse scattering scheme assumes that the speed $c(x, y, z)$ of propagation of sound, the density $\rho(x, y, z)$ and the attenuation $\gamma(x, y, z)$ are independent of the variables $x, y$, so that $c(x, y, z)=c(z)$, $\rho(x, y, z)=\rho(z), \gamma(x, y, z)=\gamma(z)$; an acoustic medium possessing these properties will be referred to as a layered medium, or layered environment.

\subsection{Statement of the Problem}

The inverse scattering problem is the problem of reconstructing the various parameters of scattering objects, such as the density, the speed of sound, and the attenuation, with the knowledge of the incident and the scattered field. Below is the formal mathematical formulation of the three-dimensional inverse scattering problem in a layered acoustic medium.

\subsubsection{The Helmholtz Equations}

The inverse scattering problem we investigate arises from the time domain wave equation

$$
\frac{\partial^{2}}{\partial^{2} t} \psi(x, t)=c^{2}(x) \cdot \rho(x) \nabla \cdot\left(\frac{1}{\rho(x)} \nabla \psi(x, t)\right),
$$

where $\psi(x, t)$ is the value of the scalar field at a point $x$ at time $t, c(x)$ is the local speed of wave propagation at a point $x$, and $\rho(x)$ is the density at a point $x$. In order to solve (1), we assume

$$
\psi(x, t)=\psi_{k}(x) e^{i k c_{0} t}
$$

where $k$ is a complex number with non-negative imaginary part, and $c_{0}$ is the speed of wave propagation outside of the scattering structure. Substituting (2) into (1), we obtain

$$
\rho(x) \nabla \cdot\left(\frac{1}{\rho(x)} \nabla \psi_{k}(x)\right)+k^{2} \cdot \frac{c_{0}^{2}}{c^{2}(x)} \psi_{k}(x)=0 .
$$

Since the inverse scattering scheme assumes that the speed $c(x, y, z)$ of propagation of sound, the density $\rho(x, y, z)$ and the attenuation $\gamma(x, y, z)$ are independent of the variables $x, y$, i.e., $c(x, y, z)=c(z), \rho(x, y, z)=\rho(z), \gamma(x, y, z)=\gamma(z),(3)$ can be rewritten by the formula

$$
\frac{\partial^{2} \psi_{k}}{\partial x^{2}}+\frac{\partial^{2} \psi_{k}}{\partial z^{2}}-\frac{1}{\rho(z)} \frac{d \rho}{d z} \cdot \frac{\partial \psi_{k}}{\partial z}+k^{2} \cdot \frac{c_{0}^{2}}{c^{2}(z)} \cdot \psi_{k}=0 .
$$


Throughout this paper, we use the notation

$$
\frac{c_{0}^{2}}{c^{2}(z)}=1+q(z)+i \cdot \gamma(z)
$$

where $q(z)$ and $\gamma(z)$ are known as potential and attenuation of the layered acoustic medium, and that $\rho, q, \gamma \in c_{0}^{2}([0,1])$, i.e., $\rho, q, \gamma$ are twice continuously differentiable everywhere, and are defined by the formulae

$$
\begin{aligned}
& \rho(x)=\rho(0) \equiv \rho_{1}, \text { for all } x \leq 0, \\
& \rho(x)=\rho(1) \equiv \rho_{2}, \text { for all } x \geq 1, \\
& q(x)=q(0) \equiv q_{1}, \text { for all } x \leq 0, \\
& q(x)=q(1) \equiv q_{2}, \text { for all } x \geq 1, \\
& \gamma(x)=\gamma(0) \equiv \gamma_{1}, \text { for all } x \leq 0, \\
& \gamma(x)=\gamma(1) \equiv \gamma_{2}, \text { for all } x \geq 1
\end{aligned}
$$

Suppose now that the angle of incidence with respect to the normal to the $x$-y plane is $\theta$, and

$$
\psi_{k}(x, z)=e^{i k x \sin \theta} \cdot \phi(z) .
$$

Substituting (12) into (4), we obtain

$$
\phi^{\prime \prime}(x, k)-\frac{\rho^{\prime}(x)}{\rho(x)} \cdot \phi^{\prime}(x, k)+k^{2} \cdot\left(1+q(x)+i \cdot \gamma(x)-\alpha^{2}\right) \cdot \phi(x, k)=0,
$$

where

$$
\alpha=\sin (\theta) \text {. }
$$

Equation (13) is the well-known scalar Helmholtz equation in layered acoustic media. For any complex $k$, we consider solutions of the Helmholtz equation $\phi_{+}(x, k)$ and $\phi_{-}(x, k)$ defined by the formulae

$$
\begin{aligned}
& \phi_{+}(x, k)=\phi_{\text {inc+ }}(x, k)+\phi_{\text {scat }+}(x, k), \\
& \phi_{-}(x, k)=\phi_{\text {inc- }}(x, k)+\phi_{\text {scat- }}(x, k),
\end{aligned}
$$

with

$$
\begin{gathered}
\phi_{i n c+}(x, k)=e^{i k \sqrt{1+q_{1}+i \gamma_{1}-\alpha^{2}} x}, \text { for all } x<1, \\
\phi_{i n c+}(x, k)=e^{i k \sqrt{1+q_{2}+i \gamma_{2}-\alpha^{2}} x}, \text { for all } x \geq 1, \\
\phi_{\text {inc- }}(x, k)=e^{-i k \sqrt{1+q_{1}+i \gamma_{1}-\alpha^{2}} x}, \text { for all } x \leq 0, \\
\phi_{\text {inc- }}(x, k)=e^{-i k \sqrt{1+q_{2}+i \gamma_{2}-\alpha^{2}} x}, \text { for all } x>0,
\end{gathered}
$$

and $\phi_{\text {scat }+}, \phi_{\text {scat- }}$ satisfying the outgoing radiation boundary conditions

$$
\phi_{\text {scat }}^{\prime}(0, k)+i k \sqrt{1+q_{1}+i \gamma_{1}-\alpha^{2}} \phi_{\text {scat } \pm}(0, k)=0
$$




$$
\phi_{\text {scat }}^{\prime}(1, k)-i k \sqrt{1+q_{2}+i \gamma_{2}-\alpha^{2}} \phi_{\text {scat }}(1, k)=0 .
$$

Combining equations $(13)-(20)$, we obtain the equations

$$
\begin{aligned}
& \phi_{\text {scat }+}^{\prime \prime}(x, k)-\frac{\rho^{\prime}(x)}{\rho(x)} \cdot \phi_{\text {scat }+}^{\prime}(x, k)+k^{2} \cdot\left(1+q(x)+i \cdot \gamma(x)-\alpha^{2}\right) \cdot \phi_{\text {scat }+}(x, k) \\
& =-\left(k^{2}\left(\left(q-q_{1}\right)+i\left(\gamma-\gamma_{1}\right)\right)-\frac{\rho^{\prime}(x)}{\rho(x)} i k \sqrt{1+q_{1}+i \gamma_{1}-\alpha^{2}}\right) \cdot e^{i k \sqrt{1+q_{1}+i \gamma_{1}-\alpha^{2}} x}, \\
& \text { for all } x<1 \text {, } \\
& \phi_{\text {scat+ }}^{\prime \prime}(x, k)-\frac{\rho^{\prime}(x)}{\rho(x)} \cdot \phi_{\text {scat }+}^{\prime}(x, k)+k^{2} \cdot\left(1+q(x)+i \cdot \gamma(x)-\alpha^{2}\right) \cdot \phi_{\text {scat }+}(x, k) \\
& =-\left(k^{2}\left(\left(q-q_{2}\right)+i\left(\gamma-\gamma_{2}\right)\right)-\frac{\rho^{\prime}(x)}{\rho(x)} i k \sqrt{1+q_{2}+i \gamma_{2}-\alpha^{2}}\right) \cdot e^{i k \sqrt{1+q_{2}+i \gamma_{2}-\alpha^{2}} x} \\
& \text { for all } x \geq 1 \text {, } \\
& \phi_{\text {scat- }}^{\prime \prime}(x, k)-\frac{\rho^{\prime}(x)}{\rho(x)} \cdot \phi_{\text {scat- }}^{\prime}(x, k)+k^{2} \cdot\left(1+q(x)+i \cdot \gamma(x)-\alpha^{2}\right) \cdot \phi_{\text {scat }-}(x, k) \\
& =-\left(k^{2}\left(\left(q-q_{1}\right)+i\left(\gamma-\gamma_{1}\right)\right)+\frac{\rho^{\prime}(x)}{\rho(x)} i k \sqrt{1+q_{1}+i \gamma_{1}-\alpha^{2}}\right) \cdot e^{-i k \sqrt{1+q_{1}+i \gamma_{1}-\alpha^{2}} x}, \\
& \text { for all } x \leq 0 \text {, } \\
& \phi_{\text {scat- }}^{\prime \prime}(x, k)-\frac{\rho^{\prime}(x)}{\rho(x)} \cdot \phi_{\text {scat- }}^{\prime}(x, k)+k^{2} \cdot\left(1+q(x)+i \cdot \gamma(x)-\alpha^{2}\right) \cdot \phi_{\text {scat }-}(x, k) \\
& =-\left(k^{2}\left(\left(q-q_{2}\right)+i\left(\gamma-\gamma_{2}\right)\right)+\frac{\rho^{\prime}(x)}{\rho(x)} i k \sqrt{1+q_{2}+i \gamma_{2}-\alpha^{2}}\right) \cdot e^{-i k \sqrt{1+q_{2}+i \gamma_{2}-\alpha^{2}} x} \text {, } \\
& \text { for all } x>0 \text {. }
\end{aligned}
$$

Combining the equations (23) - (26) with the equations (6) - (11), we observe that, for any complex $k$, there exist complex numbers $\mu_{1 \pm}(k)$ and $\mu_{0 \pm}(k)$ such that

$$
\begin{aligned}
& \phi_{\text {scat }}(x, k)=\mu_{1 \pm}(k) \cdot e^{i k \sqrt{1+q_{2}+i \gamma_{2}-\alpha^{2}} x} \text { for all } x \geq 1 \\
& \phi_{\text {scat } \pm}(x, k)=\mu_{0 \pm}(k) \cdot e^{-i k \sqrt{1+q_{1}+i \gamma_{1}-\alpha^{2}} x} \text { for all } x \leq 0 ;
\end{aligned}
$$

combining (18), (19), (27), and (28), we obtain

$$
\begin{aligned}
& \phi_{+}(x, k)=\left(1+\mu_{1+}(k)\right) \cdot e^{i k \sqrt{1+q_{2}+i \gamma_{2}-\alpha^{2}} x} \text { for all } x \geq 1, \\
& \phi_{-}(x, k)=\left(1+\mu_{0-}(k)\right) \cdot e^{-i k \sqrt{1+q_{1}+i \gamma_{1}-\alpha^{2}} x} \text { for all } x \leq 0 .
\end{aligned}
$$

Thus, for any complex $k$, the boundary value problems for $\phi_{+}, \phi_{-}$(equations (13) -(22)) are reformulated as initial value problems (equations (13), (29), and (30)). Furthermore, for any $k \in C$, coefficients $1+\mu_{1+}(k)$ and $1+\mu_{0-}(k)$ in $(29)$, (30) are both nonzero. For example, if $1+\mu_{1+}(k)=0$, then $\phi_{+}^{\prime}(1, k)=\phi_{+}(1, k)=0$, thus, due to uniqueness theorem on initial value problems, $\phi_{+}(x, k)=0$ for all $x \in R$, i.e.,

$$
\phi_{\text {scat }}(x, k)=-\phi_{\text {inc }+}(x, k)=-e^{i k \sqrt{1+q_{1}+i \gamma_{1}-\alpha^{2}} x},
$$

for all $x<1$, which contradicts (28). Similarly, we can prove that $1+\mu_{0-}(k)$ is also non-zero. 


\subsubsection{The Impedance Functions}

Denote the upper half complex plane by $C^{+}$. For any $k \in C^{+}$, we define the impedance functions $p_{+}, p_{-}:\left(R, C^{+}\right) \rightarrow C$ by the formulae

$$
\begin{gathered}
p_{+}(x, k)=\frac{\phi_{+}^{\prime}(x, k)}{i k \rho(x) \phi_{+}(x, k)}, \\
p_{-}(x, k)=\frac{\phi_{-}^{\prime}(x, k)}{-i k \rho(x) \phi_{-}(x, k)},
\end{gathered}
$$

where $\phi_{+}, \phi_{-}$are solutions of the Helmholtz equation (13) defined by (18) and (19), $\rho$ is the density of the scattering structure.

Remark 1.1 It is easy to see that the impedance functions $p_{+}, p_{-}$are independent of the nonzero coefficients $1+\mu_{1+}(k)$ and $1+\mu_{0-}(k)$ in initial conditions $(29)$, (30). Therefore, for simplicity, the initial conditions (29), (30) are reformulated as

$$
\begin{gathered}
\phi_{+}(x, k)=e^{i k \sqrt{1+q_{2}+i \gamma_{2}-\alpha^{2}} x} \text { for all } x \geq 1, \\
\phi_{-}(x, k)=e^{-i k \sqrt{1+q_{1}+i \gamma_{1}-\alpha^{2}} x} \text { for all } x \leq 0 .
\end{gathered}
$$

The solutions $\phi_{+}, \phi_{-}$as solutions of equation (13) subject to boundary conditions (34), (35) only differ from those subject to boundary conditions (29), (30) by constants.

Therefore, the inverse scattering problem for the equation (13) is stated as follows:

Suppose that the impedance function $p_{+}(0, k)$ is known for appropriately chosen frequencies $\left\{k_{j}, j=1,2, \ldots, M\right\}$, reconstruct the density $\rho(z)$, the potential $q(z)$, and the attenuation $\gamma(z)$, in the interval $[0,1]$ with the error that rapidly decreases with increasing $M$.

This paper is devoted to the construction of an algorithm for the solutions of the above problem.

\subsection{Overview}

As discussed in [3], four types of algorithms exist for the solution of inverse scattering problems.

1. Linearized inversion schemes, which approximate the inverse scattering problem by the problem of inverting the appropriately chosen linear operator (see e.g. [9]).

2. Methods based on nonlinear optimization techniques, which obtain the scattering parameters iteratively by solving a sequence of forward scattering problems (see e.g. [13]).

3. Gel'fand-Levitan and related techniques, converting the Helmholtz equation into the Schrödinger equation, and solving an inverse problem in the form of a linear Volterra integral equation for the latter (see e.g. [7]).

4. Methods based on trace formulae, which connect the high-frequency behavior of the solutions of the Helmholtz equation to the parameters of the scattering objects to be recovered (see e.g. [11]).

In this paper, we introduce an algorithm for the solution of inverse scattering problems in layered acoustic media. The procedure falls into the category 4, and is an extension of 
the procedure of [3], in the sense that while the latter recovers the parameters of a layered medium in which only the speed of sound is permitted to change, the algorithm of my thesis assumes that the speed of sound, the density, and the attenuation are variable, and recovers all three.

The inverse scattering schemes we construct are based on a collection of so-called trace formulae, and can be viewed as extension of the work started in [3], where the observation is made that (at least in layered media) it is possible to construct inverse scattering algorithms that, given a smoothly varying medium, require few measurements to reconstruct it. More specifically, given a medium whose parameters $c, \rho$, and $\gamma$ have $m \geq 1$ continuous derivatives, and data measured for all frequencies $\omega$ on the interval $[-a, a]$, the error of the reconstruction decays as $1 / a^{m-1}$ as $a \rightarrow \infty$. In this respect, the algorithm of [3] is similar to the Fourier Transform, and a strong argument is made that this is a very desirable property. While the algorithm of [3] assumes that the parameters $\rho$ and $\gamma$ are constant and the parameter $c$ depends on $z$, the schemes of this paper reconstruct $c, \rho$, and $\gamma$, provided that they only depend on the coordinate $z$.

The paper is organized as follows. In Section 2, we summarize several well-known mathematical facts to be used in the paper. In Section 3, we introduce analytical tools to be used in the construction of the algorithm. Section 4 states the algorithm in details, and a complexity analysis is included. In Section 5, several numerical examples are used to illustrate the performance of the algorithm. Finally, Section 6 contains generalizations and conclusions.

\section{Analytical Preliminaries}

In this section, we summarize several well-known mathematical facts to be used in the sections below. These facts are given without proofs, since all of these are either found in [1], [4], and [12], or easily derived from well-known results.

\subsection{Notation}

In this paper, we denote the upper half complex plane by $C^{+}$.

For any function $f: R \rightarrow R, f \in c_{0}^{m}([0,1])$ states that $f^{(m)}$ is continuous everywhere, and that $f(x)=f_{1}$ for all $x \leq 0, f(x)=f_{2}$ for all $x \geq 1$, where $f_{1}=f(0), f_{2}=f(1)$.

Suppose that for any $a>0$, the region $K(a) \subset C$ is defined by the formula

$$
K(a)=\{k|k \in C, \operatorname{Im}(k) \geq 0,| k \mid \geq a\} .
$$

\subsection{Basic Lemmas}

In this section, we introduce several basic lemmas to be used in the sections below following closely to [3].

For a function $f:[a, b] \rightarrow R^{1}$ and integer $n \geq 2$, the $n$-point trapezoidal rule $T_{n}$ is defined by the formula

$$
T_{n}(f)=h\left(\sum_{i=0}^{n-1} f(a+i h)-\left(\frac{f(a)+f(b)}{2}\right)\right)
$$


where

$$
h=\frac{b-a}{n-1}
$$

and is widely used as an approximation to the integral $\int_{a}^{b} f(x) d x$. It is second order convergent, as long as the function $f$ has two continuous derivatives on $[a, b]$. In other words, if $f \in C^{2}[a, b]$, then

$$
\int_{a}^{b} f(x) d x=T_{n}(f)+O\left(h^{2}\right) .
$$

Lemma 2.1 Suppose that $f \in c^{l}(R)$ with $l$ a nonnegative integer. Suppose further that $f^{(j)}(0)=0$ for $0 \leq j \leq l, f^{(l)}$ is absolutely continuous. Then there exists a positive number c such that

$$
\int_{0}^{x} f(t) e^{i k(x-t)} d t=-\sum_{j=1}^{l}\left(\frac{1}{2 i k}\right)^{j} f^{j-1}(x)+\left(\frac{1}{2 i k}\right)^{l+1}\left(f^{l}(x)+b(x, k)\right)
$$

with $b: R \times C^{+} \rightarrow C$ an absolutely continuous function of $x \in[0,1]$ such that

$$
|b(x, k)| \leq c,
$$

for all $x \in[0,1], k \in C^{+}$. Furthermore, if $f(x)=0$ for all $x \geq D$ with $D$ a positive number, then

$$
|b(x, k)| \leq c,
$$

for all $(x, k) \in R \times C^{+}$.

Lemma 2.2 Suppose that $a:[0,1] \rightarrow R$ and $b:[0,1] \rightarrow C$ are two continuous functions, and that $a(x)>0$, for all $x \in[0,1]$. Then for any two solutions $u$ and $v$ of the second order $O D E$

$$
\left(a(x) \phi^{\prime}(x)\right)^{\prime}+b(x) \phi(x)=0,
$$

there exists a constant $c$ such that

$$
a(x)\left(u(x) v^{\prime}(x)-v(x) u^{\prime}(x)\right)=c
$$

for all $x \in[0,1]$. Furthermore, $c \neq 0$ if and only if $u$ and $v$ are linearly independent. (The expression $W(u, v)=u(x) v^{\prime}(x)-v^{\prime}(x) u(x)$ is referred to as the Wronskian of the pair $u$, $v)$.

Lemma 2.3 (Gronwall's inequality) Suppose that $u, v, w:[0, a] \rightarrow R$ are three continuous and nonnegative functions, satisfying the inequality

$$
w(x) \leq u(x)+\int_{0}^{x} v(t) w(t) d t
$$

for all $x \in[0, a]$. Then,

$$
w(x) \leq u(x)+\int_{0}^{x} u(t) v(t) e^{\int_{t}^{x} v(\tau) d \tau} d t
$$

for all $x \in[0, a]$. 
Lemma 2.4 Suppose that $a: C \rightarrow C$ is an entire function and that $A: R \times C \rightarrow C^{n \times n}$ is an $n \times n$-matrix whose entries $a_{i, j}(x, z), i, j=1, \ldots, n$ are continuous functions of $x$ and entire functions of $z$ for all $x \in R$ Then for any $z \in C$, the differential equation

$$
y^{\prime}(x, z)=A(x, z) \cdot y(x, z)
$$

subject to the initial condition

$$
y(0)=c(z)
$$

has an unique solution $y(x, z)$ for all $x \in R$. Moreover, $y(x, z)$ is an entire function of $z$.

\subsection{Schrödinger Equation and Riccati Equation}

This section describes the basic facts about the Helmholtz equation and its connections with the Schrödinger equation and the Riccati equation in the context of scattering problem. Lemma 2.5 describes the fact that a Schrödinger equation with outgoing radiation conditions can be converted into a second kind integral equation with the Green's function of the corresponding Helmholtz equation. Lemma 2.6 gives the mathematical form of the Green's function for Helmholtz equation with outgoing radiation conditions.

Lemma 2.5 Suppose that $G_{k}:[0,1] \times[0,1] \rightarrow C$ is the Green's function of the boundary value problem

$$
\begin{aligned}
& \psi^{\prime \prime}(x, k)+k^{2} \psi(x, k)=0 \\
& \psi^{\prime}(0, k)+i k \psi(0, k)=0 \\
& \psi^{\prime}(1, k)-i k \psi(1, k)=0
\end{aligned}
$$

for any complex $k \neq 0$. Then the boundary value problem

$$
\begin{gathered}
\psi^{\prime \prime}(x, k)+\left(k^{2}+\eta(x)\right) \psi(x, k)=f(x, k) \\
\psi^{\prime}(0, k)+i k \psi(0, k)=0 \\
\psi^{\prime}(1, k)-i k \psi(1, k)=0
\end{gathered}
$$

is equivalent to a second kind integral equation

$$
\psi(x, k)=-\int_{0}^{1} G_{k}(x, t) \eta(t) \psi(t, k) d t+g(x, k)
$$

with $f, g:[0,1] \times C \rightarrow C$ and $g$ defined by the formula

$$
g(x, k)=\int_{0}^{1} G_{k}(x, t) f(t, k) d t .
$$

Lemma 2.6 For any complex $k \neq 0$, the Helmholtz equation

$$
\psi^{\prime \prime}(x, k)+k^{2} \psi(x, k)=0
$$

with the outgoing radiation conditions (21), (22) has the Green's function

$$
G_{k}(x, t)=\frac{1}{2 i k} \begin{cases}e^{i k(t-x)} & x \leq t \\ e^{i k(x-t)} & x \geq t\end{cases}
$$


The following lemma connects the solutions of the Helmholtz equation to those of Schrödinger equation via direct transform.

Lemma 2.7 Suppose that $q, \gamma, \rho: R \rightarrow R$ are $c^{2}$-functions such that $1+q(x)-\alpha^{2}>0$, $\gamma(x)>0, \rho(x)>0$, for all $x \in R$. Suppose further that the functions $n, t: R \rightarrow C$, $\eta, g: C \rightarrow C$ are defined by the formulae

$$
\begin{gathered}
n(x)=\sqrt{1+q(x)+i \gamma(x)-\alpha^{2}} \\
t(x)=\int_{0}^{x} n(\tau) d \tau \\
\eta(t)=\frac{1}{4}\left(1+q(x)+i \gamma(x)-\alpha^{2}\right)^{-3}\left(\left(2 \frac{\rho^{\prime \prime}(x)}{\rho(x)}-3\left(\frac{\rho^{\prime}(x)}{\rho(x)}\right)^{2}\right) \cdot\left(1+q(x)+i \gamma(x)-\alpha^{2}\right)^{2}\right. \\
\left.-\left(q^{\prime \prime}(x)+i \gamma^{\prime \prime}(x)\right) \cdot\left(1+q(x)+i \gamma(x)-\alpha^{2}\right)+\frac{5}{4}\left(q^{\prime}(x)+i \gamma^{\prime}(x)\right)^{2}\right) \\
g(t)=f(x) \cdot \rho^{-\frac{1}{2}}(x) \cdot\left(1+q(x)+i \gamma(x)-\alpha^{2}\right)^{-\frac{3}{4}} .
\end{gathered}
$$

Finally, suppose that the function $\phi: R \times C \rightarrow C$ satisfies the equation

$$
\phi^{\prime \prime}(x, k)-\frac{\rho^{\prime}(x)}{\rho(x)} \phi^{\prime}(x, k)+k^{2}\left(1+q(x)+i \gamma-\alpha^{2}\right) \phi(x, k)=f(x)
$$

and the function $\psi: C \times C \rightarrow C$ is defined by the formula

$$
\psi(t, k)=\rho^{-\frac{1}{2}}(x) \cdot\left(1+q(x)+i \gamma(x)-\alpha^{2}\right)^{\frac{1}{4}} \cdot \phi(x, k) .
$$

Then the function $\psi$ satisfies the Schrödinger equation

$$
\psi^{\prime \prime}(t, k)+\left(k^{2}+\eta(t)\right) \cdot \psi(t, k)=g(t) .
$$

Remark 2.1 Lemma 2.7 provides a connection between the solutions of the Helmholtz equation (63) and those of the appropriately chosen Schrödinger equation (65). This connection will be used in the following chapter as an analytical tool. However, it is not useful in numerical computations since the connection between $\eta$ and $q$ ( see equation (61)) is generally ill-conditioned.

Corollary 2.8 Suppose that under the conditions of the preceding lemma that $q, \gamma, \rho \in$ $c_{0}^{2}([0,1])$. Suppose further that the functions $\psi_{+}, \psi_{-}: C \times C \rightarrow C$ are defined by the formulae

$$
\begin{aligned}
& \psi_{+}(t, k)=\rho^{-\frac{1}{2}}(x) \cdot\left(1+q(x)+i \gamma(x)-\alpha^{2}\right)^{\frac{1}{4}} \cdot \phi_{+}(x, k), \\
& \psi_{-}(t, k)=\rho^{-\frac{1}{2}}(x) \cdot\left(1+q(x)+i \gamma(x)-\alpha^{2}\right)^{\frac{1}{4}} \cdot \phi_{-}(x, k) .
\end{aligned}
$$

Then $\psi_{+}, \psi_{-}$satisfy the ODEs

$$
\psi_{+}^{\prime \prime}(t, k)+\left(k^{2}+\eta(t)\right) \cdot \psi_{+}(t, k)=0
$$




$$
\psi_{-}^{\prime \prime}(t, k)+\left(k^{2}+\eta(t)\right) \cdot \psi_{-}(t, k)=0,
$$

subject to the boundary conditions

$$
\psi_{+}(t, k)=\xi(k) \cdot e^{i k\left(t-T_{1}\right)}
$$

for all $\operatorname{Re}(t) \geq \operatorname{Re}\left(T_{1}\right)$, and

$$
\psi_{-}(t, k)=\rho_{1}^{-\frac{1}{2}}\left(1+q_{1}-\alpha^{2}+i \gamma_{1}\right)^{\frac{1}{4}} e^{-i k t},
$$

for all $\operatorname{Re}(t) \leq 0$ with $T_{1}, \xi(k) \neq 0$ defined by the formulae

$$
\begin{gathered}
T_{1}=\int_{0}^{1} \sqrt{1+q(x)+i \gamma(x)-\alpha^{2}} d x \\
\xi(k)=\rho_{2}^{-\frac{1}{2}}\left(1+q_{2}-\alpha^{2}+i \gamma_{2}\right)^{\frac{1}{4}} e^{i k \sqrt{1+q_{2}+i \gamma_{2}-\alpha^{2}}} .
\end{gathered}
$$

Furthermore,

$$
\begin{gathered}
p_{+}(x, k)=\sqrt{1+q(x)+i \gamma(x)-\alpha^{2}} \frac{\psi_{+}^{\prime}(t, k)}{i k \rho(x) \psi_{+}(t, k)}+ \\
\frac{\frac{1}{2} \frac{\rho^{\prime}(x)}{\rho(x)}-\frac{1}{4}\left(1+q(x)+i \gamma(x)-\alpha^{2}\right)^{-1} \cdot\left(q^{\prime}(x)+i \gamma^{\prime}(x)\right)}{i k \rho(x)}, \\
p_{-}(x, k)=\sqrt{1+q(x)+i \gamma(x)-\alpha^{2}} \frac{\psi_{-}^{\prime}(t, k)}{-i k \rho(x) \psi_{-}(t, k)}- \\
\frac{1}{2} \frac{\rho^{\prime}(x)}{\rho(x)}-\frac{1}{4}\left(1+q(x)+i \gamma(x)-\alpha^{2}\right)^{-1} \cdot\left(q^{\prime}(x)+i \gamma^{\prime}(x)\right) \\
i k \rho(x)
\end{gathered}
$$

Observation 2.2 Suppose that $q, \gamma, \rho \in c_{0}^{2}([0,1])$. Then according to Lemma 2.7 and Corollary 2.8,

$$
t=\sqrt{1+q_{1}+i \gamma_{1}-\alpha^{2}} x
$$

and consequently

$$
\phi_{+}(x, k)=\sqrt{\rho_{1}} \cdot\left(1+q_{1}+i \gamma_{1}-\alpha^{2}\right)^{-\frac{1}{4}} \cdot \psi_{+}(t, k),
$$

for all $x \leq 0$. Now, suppose the function $\psi_{+}$is defined by formula (66). Defining the scattered field $\psi_{\text {scat+ }}: C \times C \rightarrow C$ by the formula

$$
\psi_{+}(t, k)=\rho_{1}^{-\frac{1}{2}} \cdot\left(\sqrt{1+q_{1}+i \gamma_{1}-\alpha^{2}}\right)^{\frac{1}{4}} \cdot\left(e^{i k t}+\psi_{\text {scat }+}(t, k)\right),
$$

we immediately obtain the Schrödinger equation

$$
\begin{aligned}
& \psi_{\text {scat+ }}^{\prime \prime}(t, k)+\left(k^{2}+\eta(t)\right) \psi_{\text {scat }+}(t, k)=\rho(x)^{-\frac{1}{2}} \cdot\left(1+q(x)+i \gamma(x)-\alpha^{2}\right)^{-\frac{3}{4}} \cdot \\
& \left(-k^{2}\left(\left(q-q_{1}\right)+i\left(\gamma-\gamma_{1}\right)\right)+\frac{\rho^{\prime}(x)}{\rho(x)} i k \sqrt{1+q_{1}+i \gamma_{1}-\alpha^{2}}\right) \cdot e^{i k \sqrt{1+q_{1}+i \gamma_{1}-\alpha^{2}} x}
\end{aligned}
$$

subject to outgoing radiation conditions (21), (22). 
The following lemma introduces the Riccati equations satisfied by the impedance functions $p_{+}, p_{-}$. They are obtained from the definitions of impedance functions (32) (33) and the Helmholtz equation (13).

Lemma 2.9 Suppose that under the conditions of the preceding lemma,

$$
\begin{aligned}
& \psi_{+}\left(x_{0}, k_{0}\right) \neq 0, \\
& \psi_{-}\left(x_{0}, k_{0}\right) \neq 0,
\end{aligned}
$$

at some point $\left(x_{0}, k_{0}\right) \in R \times C$. Then there exists a neighborhood $D$ of $\left(x_{0}, k_{0}\right)$ such that the impedance functions $p_{+}, p_{-}$satisfy the Riccati equations

$$
\begin{gathered}
p_{+}^{\prime}(x, k)=-i k \rho(x) \cdot\left(p_{+}^{2}(x, k)-\frac{1+q(x)+i \gamma(x)-\alpha^{2}}{\rho^{2}(x)}\right), \\
p_{-}^{\prime}(x, k)=i k \rho(x) \cdot\left(p_{-}^{2}(x, k)-\frac{1+q(x)+i \gamma(x)-\alpha^{2}}{\rho^{2}(x)}\right),
\end{gathered}
$$

for all $(x, k) \in D$.

Observation 2.3 Combining formulae (34), (35), we easily observe that

$$
\begin{aligned}
& p_{+}(x, k)=\frac{\sqrt{1+q_{2}+i \gamma_{2}-\alpha^{2}}}{\rho_{2}}, \text { for all } x \geq 1, \\
& p_{-}(x, k)=\frac{\sqrt{1+q_{1}+i \gamma_{1}-\alpha^{2}}}{\rho_{1}}, \text { for all } x \leq 0,
\end{aligned}
$$

for all complex $k \neq 0$.

Observation 2.4 If $\gamma(x)=0$ for all $x \in R$, it is easy to see from equations (82), (83), (84), and (85) that

$$
\begin{aligned}
& \overline{p_{+}(x, k)}=p_{+}(x,-k), \\
& \overline{p_{-}(x, k)}=p_{-}(x,-k) .
\end{aligned}
$$

for all real $k$, since $\overline{p_{+}(x, k)}$ and $p_{+}(x,-k)$ satisfy identical differential equations and boundary conditions, and the same is true for $\overline{p_{-}(x, k)}$ and $p_{-}(x,-k)$, too.

\section{$3 \quad$ Mathematical Apparatus}

In this section, we introduce analytical tools to be used in the construction of the algorithms of this paper. This section discusses the following three facts.

(A) For any $x \in R$, the impedance functions $p_{+}(x, k), p_{-}(x, k)$, defined by (32), (33), are analytic functions of $k$ in the upper half plane $C^{+}$. Furthermore,

$$
\begin{aligned}
p_{+}(x, k)= & \frac{1}{\rho(x)} \cdot \sqrt{1+q(x)+i \gamma-\alpha^{2}} \\
& -\frac{1}{i k} \cdot \frac{\rho(x) \cdot\left(q^{\prime}(x)+i \gamma^{\prime}(x)\right)-2 \cdot\left(1+q(x)+i \gamma(x)-\alpha^{2}\right) \cdot \rho^{\prime}(x)}{4 \cdot \rho^{2}(x) \cdot\left(1+q(x)+i \gamma(x)-\alpha^{2}\right)}+\left(k^{-2}\right),
\end{aligned}
$$




$$
\begin{aligned}
p_{-}(x, k) & =\frac{1}{\rho(x)} \cdot \sqrt{1+q(x)+i \gamma-\alpha^{2}} \\
& +\frac{1}{i k} \cdot \frac{\rho(x) \cdot\left(q^{\prime}(x)+i \gamma^{\prime}(x)\right)-2 \cdot\left(1+q(x)+i \gamma(x)-\alpha^{2}\right) \cdot \rho^{\prime}(x)}{4 \cdot \rho^{2}(x) \cdot\left(1+q(x)+i \gamma(x)-\alpha^{2}\right)}+O\left(k^{-2}\right),
\end{aligned}
$$

as $|k| \rightarrow \infty$ for all $x \in R, k \in C^{+}$.

(B) For any fixed $x \in R$, the difference between the impedance functions $p_{+}(x,-k)$ and $p_{-}(x, k)$ decays like a constant times $k^{-m}$, where $k \in R$, and $m$ is the smoothness of the scatterer. In other words,

$$
p_{+}(x,-k)-p_{-}(x, k)=O\left(k^{-m}\right),
$$

as $|k| \rightarrow \infty, k \in R$.

(C) For any $a>0$, and all $x \in R$,

$$
\begin{aligned}
\rho(x) \cdot\left(q^{\prime}(x)+i \gamma^{\prime}(x)\right)-2 \cdot \rho^{\prime}(x) \cdot\left(1+q(x)+i \gamma(x)-\alpha^{2}\right) \\
\quad=\frac{2}{\pi}\left(1+q(x)+i \gamma(x)-\alpha^{2}\right) \rho^{2}(x) \int_{-a}^{a}\left(p_{+}(x, k)-p_{-}(x, k)\right) d k+O\left(a^{-(m-1)}\right)
\end{aligned}
$$

where $m$ is the smoothness of the scatterer, $p_{+}(x, k)$ and $p_{-}(x, k)$ are impedance functions defined by (32) and (33), $\rho$ is the density of the scattering object, $q$ is the potential, and $\gamma$ is the attenuation. (91) is an example of a trace formula.

The proofs in this section are modeled after those in [3].

\subsection{Boundedness}

This section establishes the basic properties of the impedance functions $p_{+}, p_{-}$, defined by (32), (33). Lemma 3.1 describes the behavior of $\phi_{+}, \phi_{-}$in the vicinity of $k=0$ in the complex plane. Lemma 3.2 describes the properties of the impedance functions $p_{+}, p_{-}$near $k=0$. Lemma 3.3 shows that $\phi_{-}, \phi_{+}$are nonzero for all real $x$ and complex $k \neq 0$.

The following lemma describes the behavior of $\phi_{+}, \phi_{-}$in the vicinity of $k=0$ in the complex plane.

Lemma 3.1 Suppose that $\rho, q, \gamma \in c_{0}^{2}([0,1])$, and $A>0$ is a real number. Then, there exist positive numbers $\delta, \varepsilon$, and $\eta$ such that

$$
\begin{gathered}
\left|\phi_{+}(x, k)-1\right| \leq \varepsilon|k| \\
\left|\phi_{-}(x, k)-1\right| \leq \varepsilon|k| \\
\left|\phi_{+}^{\prime}(x, k)-i k \sqrt{1+q_{2}+i \gamma_{2}-\alpha^{2}} \cdot \frac{\rho(x)}{\rho_{2}}\right| \leq \eta|k|^{2}, \\
\left|\phi_{-}^{\prime}(x, k)+i k \sqrt{1+q_{1}+i \gamma_{1}-\alpha^{2}} \cdot \frac{\rho(x)}{\rho_{1}}\right| \leq \eta|k|^{2}, \\
\phi_{+}(x, k) \neq 0, \\
\phi_{-}(x, k) \neq 0,
\end{gathered}
$$

for all real $x \in[-A, A]$ and complex $k$ such that $|k|<\delta$. In (92)-(97), $q_{1}, q_{2}, \gamma_{1}, \gamma_{2}$, are defined in Section 2.1, $\alpha$ is define by (14), and $\phi_{ \pm}(x, k)$ is the field at $x$. 
Proof. Since the proofs of this lemma for $\phi_{+}, \phi_{+}^{\prime}$ and for $\phi_{-}, \phi_{-}^{\prime}$ are identical, we only prove it for $\phi_{-}, \phi_{-}^{\prime}$. Defining two auxiliary functions $\phi_{1}, \psi: R \times C^{+} \rightarrow C$ by the formulae

$$
\begin{gathered}
\phi_{1}(x, k)=\phi_{-}(x, k)-1, \\
\psi(x, k)=\frac{\phi_{-}^{\prime}(x, k)}{\rho(x)}+\frac{i k}{\rho_{1}} \cdot \sqrt{1+q_{1}+i \gamma_{1}-\alpha^{2}},
\end{gathered}
$$

and combining (98), (99) with (13) and initial conditions (34), (35), we obtain the linear first order ODEs

$$
\begin{aligned}
& \phi_{1}^{\prime}(x, k)=\rho(x) \psi(x, k)-i k \sqrt{1+q_{1}+i \gamma_{1}-\alpha^{2}} \frac{\rho(x)}{\rho_{1}}, \\
& \psi^{\prime}(x, k)=-\frac{k^{2}}{\rho(x)}\left(1+q(x)+i \gamma(x)-\alpha^{2}\right)\left(\phi_{1}(x, k)+1\right),
\end{aligned}
$$

subject to the initial conditions

$$
\begin{gathered}
\phi_{1}(0, k)=0, \\
\psi(0, k)=0 .
\end{gathered}
$$

We begin by showing that there exist two continuous functions $M, N: R^{+} \times R^{+} \rightarrow R^{+}$such that for any $s \in R^{+}, M(s, t), N(s, t)$ are monotonically increasing functions of $t$ and

$$
\begin{gathered}
\left|\phi_{1}(x, k)\right| \leq M(A,|k|)|k|, \\
|\psi(x, k)| \leq N(A,|k|)|k|^{2} .
\end{gathered}
$$

Integrating (100) from 0 to $x$, we have

$$
\phi_{1}(x, k)=\int_{0}^{x}\left(-i k \sqrt{1+q_{1}+i \gamma_{1}-\alpha^{2}} \rho(t) \frac{1}{\rho_{1}}+\rho(t) \psi(t, k)\right) d t,
$$

an substituting into (101) and integrating the result, we have

$$
\begin{aligned}
\psi(x, k)=-k^{2} \cdot \int_{0}^{x} & \frac{1+q(t)+i \gamma(t)-\alpha^{2}}{\rho(t)} \\
& \left(1+\int_{0}^{t}\left(\frac{-i k \sqrt{1+q_{1}+i \gamma_{1}-\alpha^{2}} \rho(\tau)}{\rho_{1}}+\rho(\tau) \psi(\tau, k)\right) d \tau\right) d t .
\end{aligned}
$$

Denoting $|\psi(x, k)|$ by $a(x, k)$, we have

$$
a(x, k) \leq c_{1}|k|^{2}\left(|x|+\frac{1}{2} c_{2} x^{2}|k|+c_{3} \int_{0}^{x}(x-t) a(t, k) d t\right),
$$

where

$$
\begin{gathered}
c_{1}=\sup _{-A<x<A}\left|\frac{1+q(x)+i \gamma(x)-\alpha^{2}}{\rho(x)}\right|, \\
c_{2}=\sup _{-A<x<A}\left|\sqrt{1+q_{1}+i \gamma_{1}-\alpha^{2}} \cdot \frac{\rho(x)}{\rho_{1}}\right|,
\end{gathered}
$$




$$
c_{3}=\sup _{-A<x<A}|\rho(x)| .
$$

Using Lemma 2.3, we rewrite (108) in the form

$$
a(x, k) \leq|k|^{2} u(x)+|k|^{4} \int_{0}^{x} u(t) v(t)\left(1+e^{-\frac{1}{2} c_{1} c_{2} k^{2}(x-t)^{2}}\right) d t
$$

where

$$
\begin{gathered}
u(t)=c_{1}|t|+\frac{1}{2} c_{1} c_{2} t^{2}|k|, \\
v(t)=c_{1} c_{3}(x-t) .
\end{gathered}
$$

Now, (105) follows immediately with $N(A,|k|)$ defined by the formula

$$
N(A,|k|)=\sup _{-A<x<A} u(x)+|k|^{2} \int_{0}^{x} u(t) v(t)\left(1+e^{-\frac{1}{2} c_{1} c_{2} k^{2}(x-t)^{2}}\right) d t .
$$

It is easily observed from (106) that

$$
\left|\phi_{1}(x, k)\right| \leq c_{2}|x||k|+c_{3} N(A,|k|)|x||k|^{2}
$$

with $c_{2}, c_{3}, N(A,|k|)$ defined by (110), (111), (115), respectively. Therefore, (104) follows immediately with $M(A,|k|)$ defined by the formula

$$
M(A,|k|)=A\left(c_{2}+c_{3} N(A,|k|)|k|\right) .
$$

Since $M(A, t)$ is a continuous, monotonically increasing function of $t$, there exists a real $\delta$ such that

$$
M(A, \delta) \cdot \delta<1
$$

Denoting $M(A, \delta)$ by $\varepsilon, N(A, \delta)$ by $\frac{\eta}{c_{3}}$, and observing that $M(A,|k|), N(A,|k|)$ are monotonically increasing functions, we have

$$
\begin{gathered}
\left|\phi_{1}(x, k)\right| \leq M(A,|k|)|k| \leq M(A, \delta)|k|=\varepsilon|k|, \\
|\psi(x, k)| \leq N(A,|k|)|k| \leq N(A, \delta)|k|=\frac{\eta}{c_{3}}|k|^{2},
\end{gathered}
$$

from which (93), (95) follow immediately.

Finally, combining (119) with (118), we obtain (97).

The following lemma describes the properties of the impedance functions $p_{+}, p_{-}$near $k=0$.

Lemma 3.2 Suppose that $\rho, q, \gamma \in c_{0}^{2}([0,1])$ and $A>0$ is a real number. Then there exists a real number $\delta>0$ such that the impedance functions $p_{+}, p_{-}$, defined by (32), (33), are continuous functions of $(x, k)$ for all real $(x, k) \in D$, where

$$
D=\{(x, k)|x \in[-A, A], k \in C, k \neq 0,| k \mid \leq \delta\} .
$$


Furthermore,

$$
\begin{aligned}
& \lim _{k \rightarrow 0} p_{+}(x, k)=\frac{\sqrt{1+q_{2}+i \gamma_{2}-\alpha^{2}}}{\rho_{2}}, \\
& \lim _{k \rightarrow 0} p_{-}(x, k)=\frac{\sqrt{1+q_{1}+i \gamma_{1}-\alpha^{2}}}{\rho_{1}},
\end{aligned}
$$

where $q_{1}, q_{2}, \gamma_{1}, \gamma_{2}, \rho_{1}, \rho_{2}$ are defined in Section 2.1, $\alpha, p_{+}, p_{-}$are defined by (14), (32), (33), perspectively.

Proof. Due to Lemma 3.1, there exists a real number $\delta>0$ such that $\phi_{+}(x, k) \neq 0$, $\phi_{-}(x, k) \neq 0$ for all real $(x, k) \in D$. Therefore, the impedance functions $p_{+}, p_{-}$are welldefined in $D$, and their continuity follows from the continuity of $\phi_{+}, \phi_{-}, \phi_{+}^{\prime}, \phi_{+}^{\prime}, \rho$, as well as their definitions (32), (33). (122), (123) are obtained via the direct application of (92)-(95) and (32), (33).

Remark 3.1 Due to Lemma 3.2, if we define $p_{+}(x, 0)=\frac{\sqrt{1+q_{2}+i \gamma_{2}-\alpha^{2}}}{\rho_{2}}$, $p_{-}(x, 0)=\frac{\sqrt{1+q_{1}+i \gamma_{1}-\alpha^{2}}}{\rho_{1}}$, then $p_{+}, p_{-}$are continuous functions even at $k=0$.

The following lemma states that $\phi_{+}, \phi_{-}, \phi_{+}^{\prime}, \phi_{-}^{\prime}$ are nonzero for all real $x$ and complex $k \neq 0$.

Lemma 3.3 For all $x \in R$ and complex $k \neq 0$,

$$
\begin{aligned}
& \phi_{+}(x, k) \neq 0, \\
& \phi_{+}^{\prime}(x, k) \neq 0, \\
& \phi_{-}(x, k) \neq 0, \\
& \phi_{-}^{\prime}(x, k) \neq 0 .
\end{aligned}
$$

Proof. Since the proofs of this lemma for $\phi_{+}, \phi_{+}^{\prime}$ and for $\phi_{-}, \phi_{-}^{\prime}$ are identical, we only prove (126) and (127). We decompose $\phi_{-}$into two parts by the formulae

$$
\begin{aligned}
\phi_{-}(x, k) & =u(x, k)+i v(x, k), \\
\phi_{-}^{\prime}(x, k) & =u^{\prime}(x, k)+i v^{\prime}(x, k),
\end{aligned}
$$

such that functions $u, v: R \times C \rightarrow C$ satisfy equation (13). Combining the initial condition (35) with (128), we obtain that

$$
\begin{aligned}
& u(x, k)=\cos \left(k \sqrt{1+q_{1}+i \gamma_{1}-\alpha^{2}} x\right), \\
& v(x, k)=\sin \left(k \sqrt{1+q_{1}+i \gamma_{1}-\alpha^{2}} x\right),
\end{aligned}
$$

for all $x \leq 0$ and $k \neq 0$. Lemma 2.2 states that the Wronskian $W(u, v)$ of the pair $u, v$ is given by

$$
W(u, v)=\sqrt{1+q_{1}+i \gamma_{1}-\alpha^{2}} \cdot k,
$$

for any $x \in R$. Therefore, for any $k \neq 0, u(x, k), v(x, k)$ can not both be zero simultaneously, nor can $u^{\prime}(x, k), v^{\prime}(x, k)$. Now, (126), (127) follows immediately given (128), (129). 


\subsection{Asymptotics and Smoothness}

The principal purpose of this section is to formulate and prove the facts (A) and (B) described in the beginning of Section 3. We begin by deriving equations (88), (89), and Lemma 3.4, assuming that such asymptotic forms exist for impedance functions. Then, we demonstrate the existence of such asymptotic expansions (Lemma 3.9), by converting the Schrödinger equation into an integral equation (Lemma 3.5) and applying the Neumann series (Lemma 3.6). Finally, the statements (A) and (B) are formulated and demonstrated by Theorems $3.10,3.12$. The following lemma yields the first two terms in the asymptotic forms of the impedance functions $p_{-}, p_{+}$.

Lemma 3.4 Suppose that impedance functions $p_{+}(x, k), p_{-}(x, k)$, defined by (32), (33), are given by the asymptotic series

$$
\begin{aligned}
& p_{+}(x, k)=a_{0}(x)+\frac{a_{1}(x)}{i k}+\frac{a_{2}(x)}{(i k)^{2}}+\cdots, \\
& p_{-}(x, k)=b_{0}(x)+\frac{b_{1}(x)}{i k}+\frac{b_{2}(x)}{(i k)^{2}}+\cdots,
\end{aligned}
$$

for large real $k$. Then,

$$
\begin{gathered}
a_{0}(x)=b_{0}(x)=\frac{1}{\rho(x)} \cdot \sqrt{1+q(x)+i \gamma-\alpha^{2}}, \\
a_{1}(x)=-b_{1}(x)=-\frac{\rho(x) \cdot\left(q^{\prime}(x)+i \gamma^{\prime}(x)\right)-2 \cdot\left(1+q(x)+i \gamma(x)-\alpha^{2}\right) \cdot \rho^{\prime}(x)}{4 \cdot \rho^{2}(x) \cdot\left(1+q(x)+i \gamma(x)-\alpha^{2}\right)},
\end{gathered}
$$

where $\rho, q, \gamma, \alpha$ are defined in (13).

Proof. It is easily observed from $(82),(83)$ that the impedance functions $p_{+}(x,-k), p_{-}(x, k)$ satisfy the same Riccati differential equation (83). Hence, we have

$$
\begin{gathered}
a_{i}(x)=b_{i}(x), \text { for all the even } i, \\
a_{i}(x)=-b_{i}(x), \text { for all the odd } i
\end{gathered}
$$

(135), (136), as well as other asymptotic coefficients $b_{i}(x), i=2,3, \ldots$, are obtained by plugging (134) into (83) and comparing terms of different orders of $k$.

The following lemma converts the Schrödinger equation (69) into an integral equation.

Lemma 3.5 Suppose, for all $x \in R$ and complex $k \neq 0$,

$$
\begin{gathered}
t(x)=\int_{0}^{x} \sqrt{1+q(\tau)-\alpha^{2}+i \gamma(\tau)} d \tau, \\
m(t, k)=e^{i k t} \psi_{-}(t, k), \\
n(t, k)=-\frac{e^{i k t}}{i k} \psi_{-}^{\prime}(t, k),
\end{gathered}
$$


where $\psi_{-}$is defined in (67), $q, \rho, \gamma \in c_{0}^{2}([0,1])$ such that $1+q(x)-\alpha^{2}>0, \gamma(x)>0, \rho(x)>0$. Suppose further that $\Gamma$ is a path defined in the complex plane such that

$$
\left\{\Gamma: t \in \Gamma, x \in R, t(x)=\int_{0}^{x} \sqrt{1+q(\tau)-\alpha^{2}+i \gamma(\tau)} d \tau\right\}
$$

Then, the differential equation (69) subject to boundary condition (71) can be converted into integral equations by the formulae

$$
\begin{gathered}
m=F_{k}(m)+\left(1+q_{1}+i \gamma_{1}-\alpha^{2}\right)^{\frac{1}{4}} \rho_{1}^{-\frac{1}{2}} \\
n(t, k)=m(t, k)-\frac{1}{2 i k} \int_{0}^{t} \eta(\tau) e^{2 i k(t-\tau)} m(\tau, k) d \tau,
\end{gathered}
$$

where

$$
F_{k}(f)(t)=\frac{1}{2 i k} \int_{0}^{t} \eta(\tau)\left(1-e^{2 i k(t-\tau)}\right) f(\tau) d \tau .
$$

Proof. Combining (69), (71) with (140), (141), we observe that $m$ satisfies the equation

$$
m^{\prime \prime}(t, k)-2 i k m^{\prime}(t, k)=-\eta(t) m(t, k),
$$

subject to the initial conditions

$$
\begin{gathered}
m(0, k)=\left(1+q_{1}+i \gamma_{1}-\alpha^{2}\right)^{\frac{1}{4}} \rho_{1}^{-\frac{1}{2}}, \\
m^{\prime}(0, k)=0 .
\end{gathered}
$$

Multiplying (146) by $e^{-2 i k t}$ and integrating the result from 0 to $t$, we have

$$
m^{\prime}(t, k)=-\int_{0}^{t} \eta(\tau) e^{2 i k(t-\tau)} m(\tau, k) d \tau \text {. }
$$

(143) is obtained immediately via integrating (149) from 0 to $t$, and equation (144) follows from (149), (143), (144).

Observation 3.2 Since $\eta(\tau)$ is continuous on the entire complex plane and zero outside of a bounded region, the functions $\eta(\tau)\left(1-e^{2 i k(t-\tau)}\right)$ and $\eta(\tau) e^{2 i k(t-\tau)}$ are bounded for all real $t, \tau$, and $k \in C^{+}$. Therefore, there exists a real number $c_{1}>0$ such that

$$
\left|F_{k}\right| \leq \frac{c_{1}}{|k|}
$$

and hence there exists a real number $A>0$ such that

$$
\left|F_{k}\right| \leq 1
$$

for all $k \in K(A)$, defined in Section 2.1. 
Remark 3.3 For complex, not purely real $t$ and $\tau$, (150) does not hold. Due to (59) and (60), it is easy to observe that $t, \tau$ are real if and only if the attenuation does not enter into our scattering problem, i.e. $\gamma(x)=0$ for all $x \in R$. Therefore, (150) holds only in the absence of attenuation $\gamma$. While our analysis applies only to the case when $\gamma=0$, numerical experiments in Section 5 indicate that our scheme still works, when the attenuation is small, i.e. $|\gamma(x)| \ll\left|1+q(x)-\alpha^{2}\right|$, for all $x \in R$, When the attenuation is relatively large, our scheme does not work.

Lemmas 3.6 and 3.7 yield the Neumann series for the integral equation (143) and an estimate of the error for using a truncation of the series.

Lemma 3.6 Suppose that $q, \rho, \gamma \in c_{0}^{\mu}([0,1]), \mu \geq 2, q^{(\mu)}, \rho^{(\mu)}, \gamma^{(\mu)}$ are absolutely continuous for all $x \in R$. Suppose further that $\Gamma$ is a path defined in the complex plane such that

$$
\left\{\Gamma: t \in \Gamma, x \in R, t(x)=\int_{0}^{x} \sqrt{1+q(\tau)-\alpha^{2}+i \gamma(\tau)} d \tau\right\} .
$$

Then for any integer $1 \leq l \leq \mu$, there exist $a_{j}: \Gamma \rightarrow R, j=1, \ldots, \mu-1, a_{\mu}: \Gamma \times C^{+} \rightarrow C$, where

$$
\frac{d^{\mu-j} a_{j}(t)}{d t^{\mu-j}}
$$

are bounded and absolutely continuous for all $t \in \Gamma, j=1, \ldots, \mu-1$, and

$$
a_{\mu}(t, k)
$$

is bounded and absolutely continuous function of $t$ for all $(t, k) \in \Gamma \times C^{+}$, such that

$$
m_{l}(t, k)=\left(1+q_{1}+i \gamma_{1}-\alpha^{2}\right)^{\frac{1}{4}} \rho_{1}^{-\frac{1}{2}}+\sum_{j=1}^{\mu-1}\left(\frac{1}{2 i k}\right)^{j} a_{j}(t)+\left(\frac{1}{2 i k}\right)^{\mu} a_{\mu}(t, k),
$$

where $m_{l}: \Gamma \times C^{+} \rightarrow C$ is defined by the formulae

$$
\begin{gathered}
m_{0}(t, k)=0 \\
m_{l}(t, k)=\left(1+q_{1}+i \gamma_{1}-\alpha^{2}\right)^{\frac{1}{4}} \rho_{1}^{-\frac{1}{2}}+F_{k}\left(m_{l-1}\right)(t, k) \\
=\left(1+q_{1}+i \gamma_{1}-\alpha^{2}\right)^{\frac{1}{4}} \rho_{1}^{-\frac{1}{2}}+\frac{1}{2 i k} \int_{0}^{t} \eta(\tau)\left(1-e^{2 i k(t-\tau)}\right) m_{l-1}(\tau, k) d \tau .
\end{gathered}
$$

Proof. We prove this lemma by induction. For $l=1$, formulae (156), (157) yield

$$
m_{1}(t, k)=\left(1+q_{1}+i \gamma_{1}-\alpha^{2}\right)^{\frac{1}{4}} \rho_{1}^{-\frac{1}{2}}
$$

for all $(t, k) \in \Gamma \times C^{+}$, which is already in the form (155) satisfying conditions (153), (154). For $l \geq 1$, assuming that $m_{l}(t, k)$ is in the form (155) satisfying conditions (153), (154), we obtain $m_{l+1}$ using (157):

$$
\begin{aligned}
m_{l+1}(t, k) & =\left(1+q_{1}+i \gamma_{1}-\alpha^{2}\right)^{\frac{1}{4}} \rho_{1}^{-\frac{1}{2}}+\frac{1}{2 i k} \int_{0}^{t} \eta(\tau)\left(1-e^{2 i k(t-\tau)}\right) m_{l}(\tau, k) d \tau \\
& =\left(1+q_{1}+i \gamma_{1}-\alpha^{2}\right)^{\frac{1}{4}} \rho_{1}^{-\frac{1}{2}}+I_{1}(t, k)+I_{2}(t, k)+I_{3}(t, k)+I_{4}(t, k)
\end{aligned}
$$


with $I_{j}: \Gamma \times C^{+} \rightarrow C, 1 \leq j \leq 4$ defined by the formulae

$$
\begin{gathered}
I_{1}(t, k)=\frac{1}{2 i k} \int_{0}^{t} \eta(\tau) d \tau+\sum_{j=2}^{\mu-1}\left(\frac{1}{2 i k}\right)^{j} \int_{0}^{t} \eta(\tau) a_{j-1}(\tau) d \tau \\
I_{2}(t, k)=-\frac{1}{2 i k} \int_{0}^{t} \eta(\tau)\left(1-e^{2 i k(t-\tau)}\right) d \tau \\
I_{3}(t, k)=-\sum_{s=2}^{\mu-1}\left(\frac{1}{2 i k}\right)^{s} \int_{0}^{t} \eta(\tau) a_{s-1}(\tau) e^{2 i k(t-\tau)} d \tau \\
\equiv-\sum_{s=2}^{\mu-1} J_{s}(t, k) \\
I_{4}(t, k)=\frac{1}{2 i k} \int_{0}^{t} \eta(\tau) a_{\mu}(\tau)\left(1-e^{2 i k(t-\tau)}\right) d \tau
\end{gathered}
$$

Clearly, we only need to show that $I_{j}, 1 \leq j \leq 4$ can be expressed in the form

$$
\sum_{j=1}^{\mu-1}\left(\frac{1}{2 i k}\right)^{j} \alpha_{j}(t)+\left(\frac{1}{2 i k}\right)^{\mu} \alpha_{\mu}(t, k)
$$

with $\alpha_{j}: \Gamma \rightarrow R, 1 \leq j \leq \mu-1$ satisfying condition (153) and $\alpha_{\mu}: \Gamma \times C^{+} \rightarrow C$ satisfying condition (154). Obviously, $I_{1}$ and $I_{4}$ are already in the form (164). We now use Lemma 2.1 to show that $I_{2}, I_{3}$ can also be expanded in the form (164). Observing that $\eta(t(x))=0$ for all $x \notin(0,1), \eta^{(\mu-2)}$ is absolutely continuous, and that $a_{j}^{(\mu-j)}, 1 \leq j \leq \mu-1$ are absolutely continuous (due to the inductive assumption), we can use formula (40) to expand $I_{2}$ and each term $J_{s}(s=1, \ldots, \mu-1)$ of $I_{3}$ as

$$
\begin{array}{r}
I_{2}(t, k)=\sum_{j=2}^{\mu-1}\left(\frac{1}{2 i k}\right)^{j} \eta^{(j-2)}(t)+\left(\frac{1}{2 i k}\right)^{\mu} b_{1}(t, k), \\
J_{s}(t, k)=\left(\frac{1}{2 i k}\right)^{s} \int_{0}^{t} \eta(\tau) a_{s-1}(\tau) e^{2 i k(t-\tau)} d \tau \\
=-\sum_{j=s+1}^{\mu-1}\left(\frac{1}{2 i k}\right)^{j} \frac{d^{(j-s-1)}}{d t^{(j-s-1)}}\left(\eta(t) a_{s-1}(t)\right)-\left(\frac{1}{2 i k}\right)^{\mu} b_{s}(t, k)
\end{array}
$$

with $b_{s}: \Gamma \times C^{+} \rightarrow C$ uniformly bounded on $\Gamma \times C^{+}$(see Lemma 2.1). Therefore, $I_{2}$ is in the form (164) due to (165), and $I_{3}$ is of the form (164) due to (166), (162). Thus, $m_{l+1}(t, k)$ can indeed be written in the form (155) satisfying conditions (153), (154). 
Lemma 3.7 Suppose that the functions $m, n, m_{\mu}, n_{\mu}: R \times C^{+} \rightarrow C$ are defined by the formulae (147), (148), (157) and

$$
n_{\mu}(t, k)=m_{\mu}(t, k)-\frac{1}{2 i k} \int_{0}^{t} \eta(\tau) e^{2 i k(t-\tau)} m_{\mu}(\tau, k) d \tau
$$

respectively. Then under the conditions of the preceding lemma, there exist positive real numbers $A, c_{1}, c_{2}, c_{3}$ such that

$$
\begin{gathered}
\left|m(t, k)-m_{\mu}(t, k)\right| \leq \frac{c_{1}}{|k|^{\mu}}, \\
\left|n(t, k)-n_{\mu}(t, k)\right| \leq \frac{c_{2}}{|k|^{\mu}},
\end{gathered}
$$

for all $(R e(t), k) \in R \times K(A)$, and

$$
\left|\frac{n(t, k)}{m(t, k)}-1\right| \leq \frac{c_{3}}{|k|^{\mu}}
$$

for all $(\operatorname{Re}(t), k) \in\left[T_{1}, \infty\right) \times K(A)$.

Proof. Due to (150), the norm of the integral operator $F_{k}$ in (157) is of the order $O\left(|k|^{-1}\right)$ for any $k \in C^{+}$, from which we observe that there exists $A \geq 0$, such that (168) is true.

Subtracting (167) from (144), we obtain

$$
\begin{aligned}
& n(t, k)-n_{\mu}(t, k) \\
& \quad=m(t, k)-m_{\mu}(t, k)-\frac{1}{2 i k} \int_{0}^{t} \eta(\tau) e^{2 i k(t-\tau)}\left(m(\tau, k)-m_{\mu}(\tau, k)\right) d \tau .
\end{aligned}
$$

Now, the estimate (169) is a direct consequence of (171), (168), and the fact stated in Observation 3.2 that in (171), the expression

$$
\frac{1}{2 i k} \eta(\tau) e^{2 i k(t-\tau)}
$$

is bounded for all $k \in K(A),-\infty<\tau \leq t<\infty$. We now prove (170) by showing that there exists a positive number $c_{3}$ such that

$$
\left|\frac{n_{\mu}(t, k)}{m_{\mu}(t, k)}-1\right| \leq \frac{c_{3}}{|k|^{\mu}}
$$

for all $(\operatorname{Re}(t), k) \in\left[\operatorname{Re}\left(T_{1}\right), \infty\right) \times K(A)$. Lemma 3.6 states that $a_{\mu}(t, k)$ in $(155)$ is bounded and absolutely continuous for all $(t, k) \in \Gamma \times C^{+}$, and $a_{j}(t)$ in (155) is also independent of $k$. Therefore, we can assume that the constant $A$ has been chosen such that for all $(R e(t), k) \in R \times K(A)$,

$$
\left|\sum_{j=1}^{\mu-1}\left(\frac{1}{2 i k}\right)^{j} a_{j}(t)+\left(\frac{1}{2 i k}\right)^{\mu} a_{\mu}(t, k)\right| \leq \frac{1}{2} \cdot\left(1+q_{1}+i \gamma_{1}-\alpha^{2}\right)^{\frac{1}{4}} \rho_{1}^{-\frac{1}{2}}
$$


or equivalently,

$$
\left|m_{\mu}(t, k)\right| \geq \frac{1}{2} \cdot\left(1+q_{1}+i \gamma_{1}-\alpha^{2}\right)^{\frac{1}{4}} \rho_{1}^{-\frac{1}{2}} .
$$

Combining (167) with (155), we obtain

$$
n_{\mu}=m_{\mu}+I_{2}(t, k)+I_{3}(t, k)+I_{5}(t, k),
$$

with $I_{2}(t, k), I_{3}(t, k)$ defined by $(161),(162)$, and $I_{5}(t, k)$ defined by the formula

$$
I_{5}(t, k)=\left(\frac{1}{2 i k}\right)^{\mu+1} \int_{0}^{t} \eta(\tau) a_{\mu}(\tau, k) e^{2 i k(t-\tau)} d \tau .
$$

Noticing that $\eta(\tau)=0$ for all $\operatorname{Re}(t) \geq \operatorname{Re}\left(T_{1}\right)$, we have

$$
\begin{aligned}
& I_{2}(t, k)=\left(\frac{1}{2 i k}\right)^{\mu} b_{1}(t, k), \\
& J_{s}(t, k)=\left(\frac{1}{2 i k}\right)^{\mu} b_{s}(t, k),
\end{aligned}
$$

for all $(\operatorname{Re}(t), k) \in\left[\operatorname{Re}\left(T_{1}\right), \infty\right) \times K(A)$, due to (165), (166). Consequently, there exists $c>0$ such that

$$
\left|I_{2}(t, k)+I_{3}(t, k)+I_{5}(t, k)\right| \leq \frac{c}{|k|^{\mu}}
$$

for all $(\operatorname{Re}(t), k) \in\left[\operatorname{Re}\left(T_{1}\right), \infty\right) \times K(A)$, since $a_{\mu}(t, k), b_{s}(t, k)$ are bounded for all $(R e(t), k) \in$ $\left[\operatorname{Re}\left(T_{1}\right), \infty\right) \times K(A)$, and $s=1, \ldots, \mu-1$.

Now, the estimate (173) is a direct consequence of (176), (180) and (175). The estimate (170) is a direct consequence of (173), (168), and (169).

The proof of the following lemma is to that of Lemma 3.7, and is therefore omitted.

Lemma 3.8 Suppose, for all $x \in R$ and complex $k \neq 0$,

$$
\begin{gathered}
t(x)=\int_{0}^{x} \sqrt{1+q(\tau)-\alpha^{2}+i \gamma(\tau)} d \tau \\
f(t, k)=e^{-i k t} \psi_{+}(t, k), \\
g(t, k)=\frac{e^{-i k t}}{i k} \psi_{+}^{\prime}(t, k),
\end{gathered}
$$

where $\psi_{+}$is defined in $(66), q, \rho, \gamma \in c_{0}^{2}([0,1])$ such that $1+q(x)-\alpha^{2}>0, \gamma(x)>0, \rho(x)>0$. Then under the conditions of the Lemma 3.7, there exist positive numbers $A, d_{3}$ such that

$$
\left|\frac{g(t, k)}{f(t, k)}-1\right| \leq \frac{d_{3}}{|k|^{\mu}}
$$

for all $(\operatorname{Re}(t), k) \in(-\infty, 0] \times K(A)$. 
Now, we are ready to show the existence of the asymptotic expansion (133), (134) for impedance functions $p_{+}, p_{-}$by converting Schrödinger equation into an integral equation (Lemma 3.5) and using the Neumann series (Lemma 3.6).

Lemma 3.9 Suppose that impedance functions $p_{+}(x, k) p_{-}(x, k)$ are defined by (32), (33), for all $x \in R, k \in C$. Then, there exist asymptotic series expansions for $p_{+}$and $p_{-}$; that $i s$, there exist sequences of complex functions $a=\left\{a_{i}: R \rightarrow C\right\}$, and $b=\left\{b_{i}: R \rightarrow C\right\}$, $i=0,1,2, \ldots$ such that $p_{+}$and $p_{-}$are asymptotic given by the series

$$
\begin{aligned}
& p_{+}(x, k)=a_{0}(x)+\frac{a_{1}(x)}{i k}+\frac{a_{2}(x)}{(i k)^{2}}+\cdots \\
& p_{-}(x, k)=b_{0}(x)+\frac{b_{1}(x)}{i k}+\frac{b_{2}(x)}{(i k)^{2}}+\cdots,
\end{aligned}
$$

as $|k| \rightarrow \infty$.

Proof. Combining (74) with (168), (169), (140), (141), (155), and (176), we obtain (186). (185) is derived similarly.

Theorems 3.10, 3.12 concern the statements (A) and (B) outlined in the beginning of Section 3.

Theorem 3.10 Suppose that $q, \rho, \gamma \in c_{0}^{2}([0,1]), 1+q(x)-\alpha^{2}>0, \gamma(x)>0, \rho(x)>0$ for all $x \in R$ and $q^{\prime \prime}, \rho^{\prime \prime}, \gamma^{\prime \prime}$ are absolutely continuous. Suppose further that

$$
D=\{(x, k) \mid x \in R, \operatorname{Im}(k) \geq 0\} .
$$

Then

(a) $\phi_{+}$and $\phi_{-}$are continuous functions of $(x, k)$ and analytic functions of $k$ for all $x \in R$ and $k \in C$;

(b) $p_{+}$and $p_{-}$are continuous functions of $(x, k)$ and analytic functions of $k$ in $D$;

(c) For all $(x, k) \in D$,

$$
\begin{aligned}
p_{+}(x, k) & =\frac{1}{\rho(x)} \cdot \sqrt{1+q(x)+i \gamma-\alpha^{2}} \\
- & \frac{1}{i k} \cdot \frac{\rho(x) \cdot\left(q^{\prime}(x)+i \gamma^{\prime}(x)\right)-2 \cdot\left(1+q(x)+i \gamma(x)-\alpha^{2}\right) \cdot \rho^{\prime}(x)}{4 \cdot \rho^{2}(x) \cdot\left(1+q(x)+i \gamma(x)-\alpha^{2}\right)}+O\left(k^{-2}\right), \\
p_{-}(x, k) & =\frac{1}{\rho(x)} \cdot \sqrt{1+q(x)+i \gamma-\alpha^{2}} \\
+ & \frac{1}{i k} \cdot \frac{\rho(x) \cdot\left(q^{\prime}(x)+i \gamma^{\prime}(x)\right)-2 \cdot\left(1+q(x)+i \gamma(x)-\alpha^{2}\right) \cdot \rho^{\prime}(x)}{4 \cdot \rho^{2}(x) \cdot\left(1+q(x)+i \gamma(x)-\alpha^{2}\right)}+O\left(k^{-2}\right),
\end{aligned}
$$


Proof. We only give the proof for $\phi_{-}, p_{-}$since the proof for $\phi_{+}, p_{+}$is very similar. We introduce two auxiliary functions $\phi_{1}, \phi_{2}: R \times C^{+} \rightarrow C$ by the formulae

$$
\begin{gathered}
\hat{\phi}(x, k)=\phi_{-}(x, k)-1, \\
\check{\phi}(x, k)=\frac{\phi_{-}^{\prime}(x, k)}{\rho(x)}+\frac{i k}{\rho_{1}} \cdot \sqrt{1+q_{1}+i \gamma_{1}-\alpha^{2}},
\end{gathered}
$$

and combining (190), (191) with (13) and initial conditions (34), (35), we obtain the linear first order ODEs

$$
\begin{aligned}
& \hat{\phi}^{\prime}(x, k)=\rho(x) \check{\phi}(x, k)-i k \sqrt{1+q_{1}+i \gamma_{1}-\alpha^{2}} \frac{\rho(x)}{\rho_{1}}, \\
& \check{\phi}^{\prime}(x, k)=-\frac{k^{2}}{\rho(x)}\left(1+q(x)+i \gamma(x)-\alpha^{2}\right)(\hat{\phi}(x, k)+1),
\end{aligned}
$$

subject to the initial conditions

$$
\begin{aligned}
& \hat{\phi}(0, k)=0, \\
& \check{\phi}(0, k)=0 .
\end{aligned}
$$

According to Lemma 2.4, $\hat{\phi}, \check{\phi}$ are continuous functions of $x$ and analytic functions of $k$ for all $x \in R$ and $k \in C$, from which part (a) follows immediately. Similarly, we obtain part (b) by combining part (a) with (33) and the fact that $\phi_{-}(x, k) \neq 0$ for all $(x, k) \in D$ (see Lemma 3.3). The expansion (189) follows immediately from Lemmas 3.9 and 3.4.

Corollary 3.11 Under the conditions of the preceding theorem, there exist positive number $c_{1}, c_{2}$ such that

$$
\begin{aligned}
& \left|e^{i k \int_{t}^{x} p_{+}(\tau, k) \rho(\tau) d \tau}\right| \leq c_{1}, \\
& \left|e^{i k \int_{t}^{x} p_{-}(\tau, k) \rho(\tau) d \tau}\right| \leq c_{2},
\end{aligned}
$$

for all $t, x \in[0,1], k \in R$, or for all $0 \leq t \leq x \leq 1, k \in C^{+}$.

Proof. Due to parts (b) and (c) of Theorem 3.10, the real part of the functions

$$
\begin{aligned}
& \operatorname{Re}\left(i k \int_{t}^{x} p_{+}(\tau, k) d \tau\right) \leq c_{3}, \\
& \operatorname{Re}\left(i k \int_{t}^{x} p_{-}(\tau, k) d \tau\right) \leq c_{4},
\end{aligned}
$$

where $c_{3}$ and $c_{4}$ does not depend on $t$ or $x$ for $t, x \in[0,1], k \in R$, or for all $0 \leq t \leq x \leq 1$, from which (196) (197) follow immediately. 
Theorem 3.12 Suppose that $q, \gamma, \rho \in c_{0}^{m}([0,1]), m \geq 2, q^{(m)}, \rho^{(m)}, \gamma^{(m)}$ are absolutely continuous and $1+q(x)-\alpha^{2}>0, \gamma(x)>0, \rho(x)>0$ for all $x \in R$. Then there exists $a$ positive real number a such that

$$
\left|p_{+}(x,-k)-p_{-}(x, k)\right| \leq \frac{a}{|k|^{m}}
$$

for all $(x, k) \in R \times C^{+}$

Proof. Due to (74), (75), we obtain

$$
p_{+}(x,-k)-p_{-}(x, k)=\sqrt{1+q(x)+i \gamma(x)-\alpha^{2}} \cdot \frac{1}{-i k \rho(x)} \cdot\left(\frac{\psi_{+}^{\prime}(t,-k)}{\psi_{+}(t,-k)}-\frac{\psi_{-}^{\prime}(t, k)}{\psi_{-}(t, k)}\right) .
$$

Combining Lemmas 3.7, (34), 3.8 and (35) yields that (201) is true for all $x \notin(0,1)$. In order to prove the theorem for $x \in(0,1)$, we observe that $p_{+}(x,-k)$ and $p_{-}(x, k)$ obey the same Riccati equation (83) due to (82) and (83). The difference $s(x, k)=p_{+}(x,-k)-p_{-}(x, k)$ satisfies the ODE

$$
s^{\prime}(x, k)=i k \rho(x)\left(p_{+}(x,-k)+p_{-}(x, k)\right) s(x, k) .
$$

Clearly, the solution to (202) is

$$
s(x, k)=e^{i k \int_{0}^{x}\left(p_{+}(t,-k)+p_{-}(t, k)\right) \rho(t) d t} s(0, k) .
$$

(196) and (197) show that there exists constant $b>0$ such that

$$
\left|e^{i k \int_{0}^{x}\left(p_{+}(t,-k)+p_{-}(t, k)\right) \rho(t) d t}\right|<b
$$

for all $(x, k) \in[0,1] \times R$. Due to $(85),(74)$, and Lemma 3.8, there exists a positive number $c$ such that for all $k \in R$,

$$
|s(0, k)|=\left|p_{+}(0,-k)-p_{-}(0, k)\right|=\left|p_{+}(0,-k)-\frac{\sqrt{1+q_{1}+i \gamma_{1}-\alpha^{2}}}{\rho_{1}}\right| \leq \frac{c}{|k|^{m}} .
$$

Now, (200) for $x \in(0,1)$ follows immediately from (203), (205). Thus, we have (200) for all $(x, k) \in R \times C^{+}$.

\subsection{Trace Formula}

In this section, we prove Theorem 3.13, which is the principal analytical tool of this paper. Theorem 3.13 describes what are known as the trace formulae for the impedance functions $p_{+}, p_{-}$in the context of varying density, speed of propagation, and attenuation.

Theorem 3.13 (Trace Formula). Suppose that $q, \rho, \gamma \in c_{0}^{m}([0,1]), m \geq 2, q^{(m)}, \gamma^{(m)}$, $\rho^{(m)}$ are absolutely continuous and $1+q(x)-\alpha^{2}>0, \gamma(x)>0, \rho(x)>0$ for all $x \in R$. Then,

$$
\begin{array}{r}
\rho(x) \cdot\left(q^{\prime}(x)+i \gamma^{\prime}(x)\right)-2 \cdot \rho^{\prime}(x) \cdot\left(1+q(x)+i \gamma(x)-\alpha^{2}\right) \\
=\frac{2}{\pi}\left(1+q(x)+i \gamma(x)-\alpha^{2}\right) \rho^{2}(x) \int_{-\infty}^{\infty}\left(p_{+}(x, k)-p_{-}(x, k)\right) d k .
\end{array}
$$


Moreover, there exists a positive number c such that

$$
\begin{aligned}
& \mid \rho(x) \cdot\left(q^{\prime}(x)+i \gamma^{\prime}(x)\right)-2 \cdot \rho^{\prime}(x) \cdot\left(1+q(x)+i \gamma(x)-\alpha^{2}\right) \\
& \quad-\frac{2}{\pi}\left(1+q(x)+i \gamma(x)-\alpha^{2}\right) \rho^{2}(x) \int_{-a}^{a}\left(p_{+}(x, k)-p_{-}(x, k)\right) d k \mid \leq \frac{c}{a^{(m-1)}}
\end{aligned}
$$

for all $x \in R, a>0$.

Proof. Due to part (C) of Theorem 3.10, there exists $c>0$ such that

$$
\begin{aligned}
& \mid\left(p_{+}(x, k)-p_{-}(x, k)\right)- \\
& \quad\left(-\frac{1}{i k} \cdot \frac{\rho(x) \cdot\left(q^{\prime}(x)+i \gamma^{\prime}(x)\right)-2 \cdot\left(1+q(x)+i \gamma(x)-\alpha^{2}\right) \cdot \rho^{\prime}(x)}{2 \cdot \rho^{2}(x) \cdot\left(1+q(x)+i \gamma(x)-\alpha^{2}\right)}\right) \mid \leq \frac{c}{|k|^{2}}
\end{aligned}
$$

for all $(x, k) \in R \times C^{+}$. Denoting by $\Upsilon$ the upper half circle of radius $A$, with clockwise orientation, in the complex $k$-plane, i.e.,

$$
\Upsilon=\left\{k\left|k \in C^{+},\right| k \mid=A\right\},
$$

and noting that $p_{+}-p_{-}$is an analytical function of $k \in C^{+}$, we obtain

$$
\int_{-A}^{A}\left(p_{+}(x, k)-p_{-}(x, k)\right) d k=\int_{\Upsilon}\left(p_{+}(x, k)-p_{-}(x, k)\right) d k .
$$

Substituting (208) into (210), we have

$$
\begin{array}{r}
\frac{2}{\pi}\left(1+q(x)+i \gamma(x)-\alpha^{2}\right) \rho^{2}(x) \cdot \int_{-A}^{A}\left(p_{+}(x, k)-p_{-}(x, k)\right) d k= \\
\rho(x) \cdot\left(q^{\prime}(x)+i \gamma^{\prime}(x)\right)-2 \cdot \rho^{\prime}(x) \cdot\left(1+q(x)+i \gamma(x)-\alpha^{2}\right)+O\left(k^{-1}\right)
\end{array}
$$

from which (206) follows immediately. In order to prove (207), we rewrite (206) as

$$
\begin{array}{r}
\rho(x) \cdot\left(q^{\prime}(x)+i \gamma^{\prime}(x)\right)-2 \cdot \rho^{\prime}(x) \cdot\left(1+q(x)+i \gamma(x)-\alpha^{2}\right)= \\
\frac{2}{\pi}\left(1+q(x)+i \gamma(x)-\alpha^{2}\right) \rho^{2}(x) \cdot \int_{-a}^{a}\left(p_{+}(x, k)-p_{-}(x, k)\right) d k+I(a)
\end{array}
$$

with $I(a)$ given by the formula

$$
I(a)=\frac{2}{\pi}\left(1+q(x)+i \gamma(x)-\alpha^{2}\right) \rho^{2}(x) \cdot\left(\int_{-\infty}^{-a}+\int_{a}^{\infty}\right)\left(p_{+}(x, k)-p_{-}(x, k)\right) d k .
$$

Due to the symmetry of the integrals in (213), we have

$$
I(a)=\frac{2}{\pi}\left(1+q(x)+i \gamma(x)-\alpha^{2}\right) \rho^{2}(x) \cdot\left(\int_{-\infty}^{-a}+\int_{a}^{\infty}\right)\left(p_{+}(x,-k)-p_{-}(x, k)\right) d k,
$$

and using (200), we obtain a constant $c$ such that

$$
|I(a)| \leq \frac{c}{a^{(m-1)}}
$$

from which (207) follows immediately. 
Remark 3.4 As there are two unknowns in (206), (view $q(x)+i \gamma(x)$ as one complex unknown, $\rho(x)$ as the other), at least two $\alpha$ 's are needed in order to solve $\rho^{\prime}, q^{\prime}, r^{\prime}$ in (206). In particular, we obtain the trace formulae

$$
\begin{gathered}
\rho^{\prime}(x)=\frac{\operatorname{Re}\left(F\left(\alpha_{1}\right)\right)-\operatorname{Re}\left(F\left(\alpha_{2}\right)\right)}{2\left(\alpha_{1}^{2}-\alpha_{2}^{2}\right)} \\
q^{\prime}(x)=\frac{1}{\rho(x)\left(\alpha_{1}^{2}-\alpha_{2}^{2}\right)}\left(\operatorname{Re}\left(F\left(\alpha_{1}\right)\right)\left(1+q(x)-\alpha_{2}^{2}\right)-\operatorname{Re}\left(F\left(\alpha_{2}\right)\right)\left(1+q(x)-\alpha_{1}^{2}\right)\right) \\
\gamma^{\prime}(x)=\frac{\operatorname{Im}\left(F\left(\alpha_{1}\right)\right) \cdot\left(\alpha_{1}^{2}-\alpha_{2}^{2}\right)+\gamma(x) \cdot\left(\operatorname{Re}\left(F\left(\alpha_{1}\right)\right)-\operatorname{Re}\left(F\left(\alpha_{2}\right)\right)\right)}{\rho(x)\left(\alpha_{1}^{2}-\alpha_{2}^{2}\right)}
\end{gathered}
$$

with

$$
F(\alpha)=\frac{2}{\pi}\left(1+q(x)+i \gamma(x)-\alpha^{2}\right) \rho^{2}(x) \int_{-a}^{a}\left(p_{+}(x, k)-p_{-}(x, k)\right) d k,
$$

for the case of two $\alpha$ 's. Multiple (more than two) choices of $\alpha$ 's would lead to an overdetermined complex linear system, and thus can be used to control the effects of noise.

\section{The Algorithm}

This section describes the algorithm of the present paper and gives details about its implementation and computational costs.

\subsection{Description of the Algorithm}

In this section, we describe a reconstruction algorithm for the scalar Helmholtz equation in layered acoustic media

$$
\phi_{ \pm}^{\prime \prime}(x, k)-\frac{\rho^{\prime}(x)}{\rho(x)} \cdot \phi_{ \pm}^{\prime}(x, k)+k^{2} \cdot\left(1+q(x)+i \cdot \gamma(x)-\alpha^{2}\right) \cdot \phi_{ \pm}(x, k)=0,
$$

subject to the initial conditions

$$
\begin{gathered}
\phi_{+}(x, k)=e^{i k \sqrt{1+q_{2}+i \gamma_{2}-\alpha^{2}} x} \text { for all } x \geq 1, \\
\phi_{-}(x, k)=e^{-i k \sqrt{1+q_{1}+i \gamma_{1}-\alpha^{2}} x} \text { for all } x \leq 0 .
\end{gathered}
$$

In (220) - (222), $x$ is a real number, $k$ is a complex number in the upper half plane, $\alpha$ is the sine of the angle of incidence with respect to the normal to the interface of layers, $\phi_{+}$and $\phi_{-}$are the scalar fields associated with right-going and left-going waves, respectively; the parameters to be recovered in this algorithm are the density $\rho$, potential $q$, and attenuation $\gamma$ of the layered media. We assume $\rho, q, \gamma \in c_{0}^{m}([0,1])$, i.e., $\rho, q, \gamma$ have $m$ continuous derivatives everywhere, and are defined by equations (6) - (11).

As discussed in Sections 2 and 3, in order to reconstruct parameters $\rho, q, \gamma$, we consider a system of integro-differential equations

$$
p_{+}^{\prime}(x, k)=-i k \rho(x) \cdot\left(p_{+}^{2}(x, k)-\frac{1+q(x)+i \gamma(x)-\alpha^{2}}{\rho^{2}(x)}\right)
$$




$$
\begin{gathered}
p_{-}^{\prime}(x, k)=i k \rho(x) \cdot\left(p_{-}^{2}(x, k)-\frac{1+q(x)+i \gamma(x)-\alpha^{2}}{\rho^{2}(x)}\right) \\
\rho^{\prime}(x)=\frac{\operatorname{Re}\left(F\left(\alpha_{1}\right)\right)-\operatorname{Re}\left(F\left(\alpha_{2}\right)\right)}{2\left(\alpha_{1}^{2}-\alpha_{2}^{2}\right)} \\
q^{\prime}(x)=\frac{1}{\rho(x)\left(\alpha_{1}^{2}-\alpha_{2}^{2}\right)}\left(\operatorname{Re}\left(F\left(\alpha_{1}\right)\right)\left(1+q(x)-\alpha_{2}^{2}\right)-\operatorname{Re}\left(F\left(\alpha_{2}\right)\right)\left(1+q(x)-\alpha_{1}^{2}\right)\right) \\
\gamma^{\prime}(x)=\frac{\operatorname{Im}\left(F\left(\alpha_{1}\right)\right) \cdot\left(\alpha_{1}^{2}-\alpha_{2}^{2}\right)+\gamma(x) \cdot\left(\operatorname{Re}\left(F\left(\alpha_{1}\right)\right)-\operatorname{Re}\left(F\left(\alpha_{2}\right)\right)\right)}{\rho(x)\left(\alpha_{1}^{2}-\alpha_{2}^{2}\right)}
\end{gathered}
$$

with

$$
F(\alpha)=\frac{2}{\pi}\left(1+q(x)+i \gamma(x)-\alpha^{2}\right) \rho^{2}(x) \int_{-a}^{a}\left(p_{+}(x, k)-p_{-}(x, k)\right) d k,
$$

subject to the initial conditions

$$
\begin{gathered}
p_{+}(0, k)=p_{0}(k) \\
p_{-}(0, k)=\frac{\sqrt{1+q_{1}+i \gamma_{1}-\alpha^{2}}}{\rho_{1}} \\
\rho(0)=\rho_{1} \\
q(0)=q_{1} \\
\gamma(0)=\gamma_{1} .
\end{gathered}
$$

In $(223),(224)$, the impedance functions $p_{+}, p_{-}:\left(R, C^{+}\right) \rightarrow C$ are defined by the formulae

$$
\begin{gathered}
p_{+}(x, k)=\frac{\phi_{+}^{\prime}(x, k)}{i k \rho(x) \phi_{+}(x, k)}, \\
p_{-}(x, k)=\frac{\phi_{-}^{\prime}(x, k)}{-i k \rho(x) \phi_{-}(x, k)} .
\end{gathered}
$$

(223) and (224) are Riccati equations obtained directly from the Helmholtz equation (220) and the definitions of impedance functions (234), (235); equations (225), (226), (227) are known as trace formulae, connecting the Fourier components of the solutions of the Helmholtz equation to the parameters of the scattering objects to be recovered.

Our reconstruction algorithm for the inverse scattering problem in layered acoustic media amounts to solving numerically a self-contained set of ODEs, i.e., (223) - (227), subject to the initial conditions $(229)-(233)$. In this paper, the ODE solver from [8] is used.

As we shall see in Section 5, for sufficiently large $a$, the system of ODEs (223) - (227) has a unique solution for all $x \in[0,1]$, and this solution is stable with respect to small perturbations of the initial data $p_{0}(k)$. The inversion algorithm is $(m-1)^{t h}$-order convergent for all three parameters $\rho, q, \gamma$ to be recovered, where $m$ is the smoothness of $\rho, q$, and $\gamma$. 


\subsection{Implementation}

In implementing the algorithm stated above, the integral

$$
\int_{-a}^{a}\left(p_{+}(x, k)-p_{-}(x, k)\right) d k
$$

in (228) is approximated by the trapezoidal rule $T_{n}$, i.e.,

$$
\begin{array}{r}
T_{n}\left(p_{+}(x, k)-p_{-}(x, k)\right)=h \sum_{j=-M+1}^{M-1}\left(p_{+}\left(x, k_{j}\right)-p_{-}\left(x, k_{j}\right)\right) \\
+\frac{h}{2}\left(\left(p_{+}(x,-a)-p_{-}(x,-a)\right)+\left(p_{+}(x, a)-p_{-}(x,-)\right)\right),
\end{array}
$$

with $h=a / M, k_{j}=j h, j=-M, \ldots, M$. Thus, the system of integro-differential equations $(223)-(227)$ subject to initial conditions (229) - (233) is converted into a system of $8 M+7$ ODEs

$$
\begin{gathered}
p_{+}^{\prime}\left(x, k_{j}\right)=-i k_{j} \rho(x) \cdot\left(p_{+}^{2}\left(x, k_{j}\right)-\frac{1+q(x)+i \gamma(x)-\alpha^{2}}{\rho^{2}(x)}\right) \\
p_{-}^{\prime}\left(x, k_{j}\right)=i k_{j} \rho(x) \cdot\left(p_{-}^{2}\left(x, k_{j}\right)-\frac{1+q(x)+i \gamma(x)-\alpha^{2}}{\rho^{2}(x)}\right) \\
\rho^{\prime}(x)=\frac{\operatorname{Re}\left(F\left(\alpha_{1}\right)\right)-\operatorname{Re}\left(F\left(\alpha_{2}\right)\right)}{2\left(\alpha_{1}^{2}-\alpha_{2}^{2}\right)} \\
q^{\prime}(x)=\frac{1}{\rho(x)\left(\alpha_{1}^{2}-\alpha_{2}^{2}\right)}\left(\operatorname{Re}\left(F\left(\alpha_{1}\right)\right)\left(1+q(x)-\alpha_{2}^{2}\right)-\operatorname{Re}\left(F\left(\alpha_{2}\right)\right)\left(1+q(x)-\alpha_{1}^{2}\right)\right) \\
\gamma^{\prime}(x)=\frac{\operatorname{Im}\left(F\left(\alpha_{1}\right)\right) \cdot\left(\alpha_{1}^{2}-\alpha_{2}^{2}\right)+\gamma(x) \cdot\left(\operatorname{Re}\left(F\left(\alpha_{1}\right)\right)-\operatorname{Re}\left(F\left(\alpha_{2}\right)\right)\right)}{\rho(x)\left(\alpha_{1}^{2}-\alpha_{2}^{2}\right)}
\end{gathered}
$$

with

$$
F(\alpha)=\frac{2}{\pi}\left(1+q(x)+i \gamma(x)-\alpha^{2}\right) \rho^{2}(x) \cdot T_{2 M+1}\left(p_{+}(x, k)-p_{-}(x, k)\right),
$$

and subject to the initial conditions

$$
\begin{gathered}
p_{+}\left(0, k_{j}\right)=p_{0}\left(k_{j}\right) \\
p_{-}\left(0, k_{j}\right)=\frac{\sqrt{1+q_{1}+i \gamma_{1}-\alpha^{2}}}{\rho_{1}} \\
\rho(0)=\rho_{1} \\
q(0)=q_{1} \\
\gamma(0)=\gamma_{1}
\end{gathered}
$$


Remark 4.1 In all the numerical examples in Section 5, the values of the initial impedance functions $p_{0}\left(k_{j}\right), j=-M, \ldots, M$, required for the reconstruction scheme, are provided by solving forward scattering problems, namely, $4 M+1$ independent ODEs

$$
\phi^{\prime \prime}\left(x, k_{j}\right)-\frac{\rho^{\prime}(x)}{\rho(x)} \cdot \phi^{\prime}\left(x, k_{j}\right)+k_{j}^{2} \cdot\left(1+q(x)+i \cdot \gamma(x)-\alpha^{2}\right) \cdot \phi\left(x, k_{j}\right)=0,
$$

subject to the boundary conditions

$$
\phi_{+}\left(x, k_{j}\right)=e^{i k_{j} \sqrt{1+q_{2}+i \gamma_{2}-\alpha^{2}} x} \text { for all } x \geq 1,
$$

for $k_{j}=j \cdot \frac{a}{M}, j=-M, \ldots, M$ and $\alpha=\alpha_{1}, \alpha_{2}$. Again, we used the ODE solver in [8].

Remark 4.2 Due to Observation 2.3, for all $x, k \in R$,

$$
\begin{aligned}
& \overline{p_{+}(x, k)}=p_{+}(x,-k), \\
& \overline{p_{-}(x, k)}=p_{-}(x,-k),
\end{aligned}
$$

thus, the integral $\int_{-a}^{a}\left(p_{+}(x, k)-p_{-}(x, k)\right) d k$ in (228) is equal to

$$
2 \cdot \int_{0}^{a} \operatorname{Re}\left(p_{+}(x, k)-p_{-}(x, k)\right) d k .
$$

Therefore, the dimensions of the system of ODEs we consider (see equations (238) - (242)) is reduced to $4 M+7$ from $8 M+7$.

Remark 4.3 According to Lemma 3.2 and Remark 3.1, impedance functions $p_{+}(x, k)$, $p_{-}(x, k)$ are continuous in the vicinity of $k=0$. This allowed us to use Lagrange interpolation to get the values of $p_{+}(x, k), p_{-}(x, k)$ at $k=0$.

\subsection{Complexity Analysis}

The time cost of the inverse scheme is of the order $O\left(N_{k} \cdot N_{z}\right)$, where $N_{k}$ is the number of measurements in the frequency domain, and $N_{z}$ is the number of nodes in the space domain, since the computational cost for the ODE solver we use is proportional to the dimension of the ODE system ( $N_{k}$ in our case) and the number of discretization points in the space domain $\left(N_{z}\right)$.

Further, the storage requirements of the algorithm are also determined by $N_{k}$ and $N_{z}$, and is of the form

$$
S=O\left(K \cdot N_{k}\right)+O\left(N_{z}\right),
$$

where $K$ is a constant determined by the precision required by the ODE solver in [8]. For single precision, $K=22$; for double precision, $K=60$. 


\section{$5 \quad$ Numerical Examples}

The algorithm of Section 4 has been implemented in Fortran 77 in double precision. In this section, we illustrate the performance of the scheme as applied to several different classes of scattering objects, from Gaussian to discontinuous staircase-shaped ones. The experiments were carried out on a $2.8 \mathrm{GHz}$ Pentium D desktop with $2 \mathrm{~Gb}$ of RAM and an L2 cache of $1 \mathrm{Mb}$. The calculations reported in Examples 1 and 2 were carried out with a requested accuracy of $10^{-16}$ in the ODE solver; the calculations reported in Examples 3 - 7 were carried out with a requested accuracy of $10^{-7}$.

In Examples 1 - 3, the scatterers satisfy the smoothness conditions of Theorem 3.3. In Examples $4-4.4$, the scatterers violate the smoothness conditions mildly, as the scatterers are continuous but their derivatives are not continuous. In Examples $5-6$, the scatterers strongly violate the smoothness conditions, as those scatterers are discontinuous. In Example 7, we performed a crude test of stability of the algorithm by truncating the input scattering data $p_{+}(-1, k)$ and using it in the reconstruction algorithm. The headings of the Tables are defined as follows:

$a$ is the largest frequency used in the algorithm;

$h_{k}$ is the step size in the discretization of frequency;

$N_{x}$ is the number of discretization points in $[-1,1]$;

$E_{\rho}^{2}, E_{q}^{2}, E_{\gamma}^{2}$ are the relative $L^{2}$ norms of error of $\rho, q, \gamma$;

$E_{\rho}^{\infty}, E_{q}^{\infty}, E_{\gamma}^{\infty}$ are the relative maximum norms of error of $\rho, q, \gamma$;

$t_{C P U}$ is the CPU time required in seconds.

Example 1: In this example, we reconstruct scattering parameters $\rho, q$, and $\gamma$ of the Gaussian distribution given by the formulae

$$
\begin{gathered}
\rho(x)=1000+500 \cdot e^{-40 x^{2}}, \\
q(x)=e^{-40 x^{2}}, \\
\gamma(x)=0.01+0.01 \cdot e^{-40 x^{2}} .
\end{gathered}
$$

This is an example of scatterer whose $\rho, q$, and $\gamma$ are in $C_{0}^{\infty}$ in the interval $[-1,1]$ up to double precision. Tables 1 and 2 illustrate the numerical behavior of the reconstruction algorithm, and Figure 1 contains graphs of the exact and the recovered $\rho, q, \gamma \in C_{0}^{\infty}$ (they are almost indistinguishable in the graph) and the input impedance function $p_{+}(-1, k)$. In this example, the algorithm converges extremely rapidly as we would expect.

Example 2 : In this example, we reconstruct a more complicated scattering object given by the formulae

$$
\begin{gathered}
\rho(x)=1000+1000 \cdot e^{-40 x^{2}} \cdot \cos (30 x) \cdot \frac{e^{5 x}-e^{-5 x}}{e^{5 x}+e^{-5 x}} \\
q(x)=e^{-40 x^{2}} \cdot \cos (20 x) \cdot \frac{e^{5 x}-e^{-5 x}}{e^{5 x}+e^{-5 x}} \\
\gamma(x)=0.01+0.004 \cdot e^{-40 x^{2}} \cdot \sin (10 x) \cdot \frac{e^{5 x}-e^{-5 x}}{e^{5 x}+e^{-5 x}}
\end{gathered}
$$



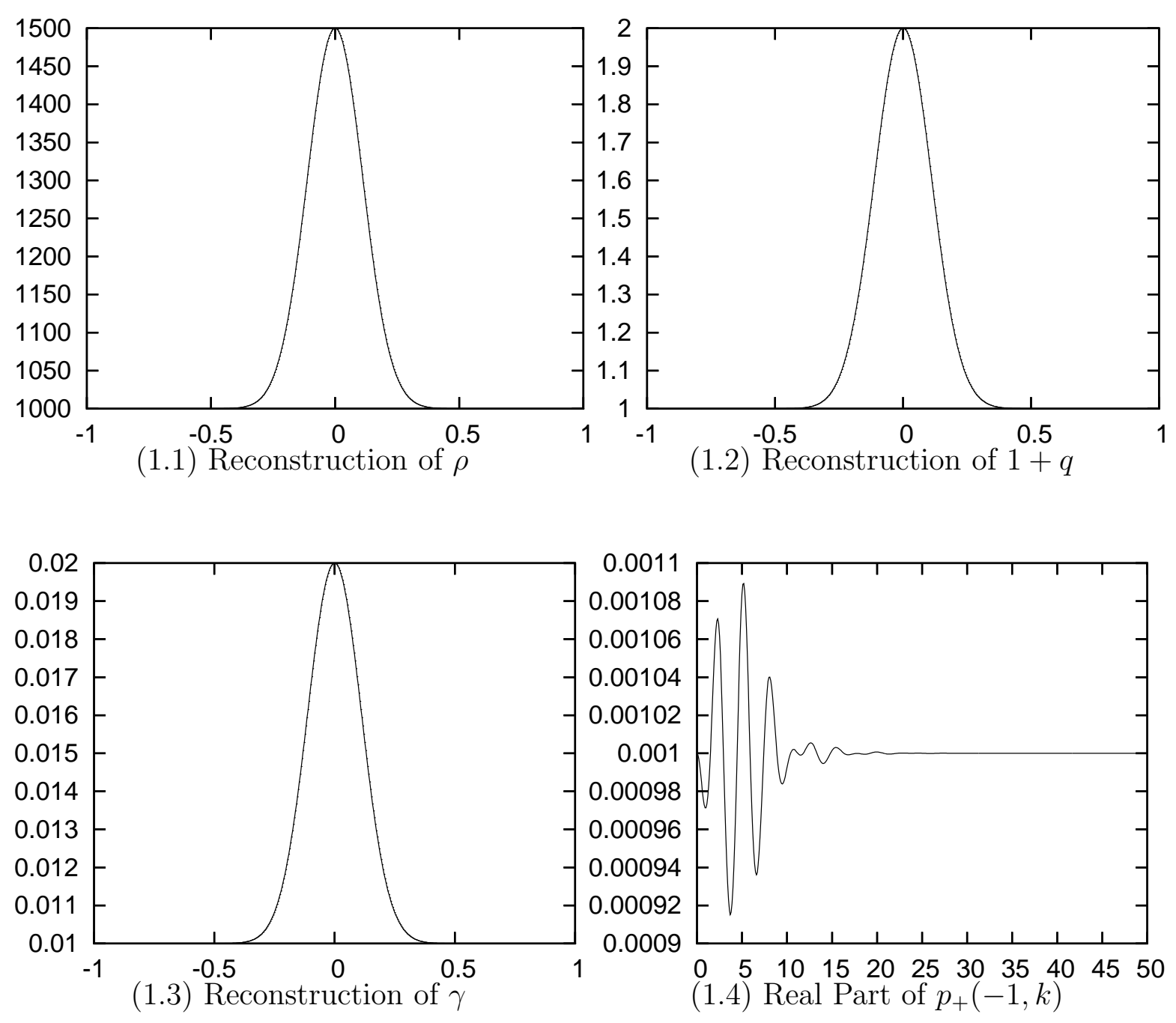

Figure 1: Reconstruction of Example 1 with $a=50$ 

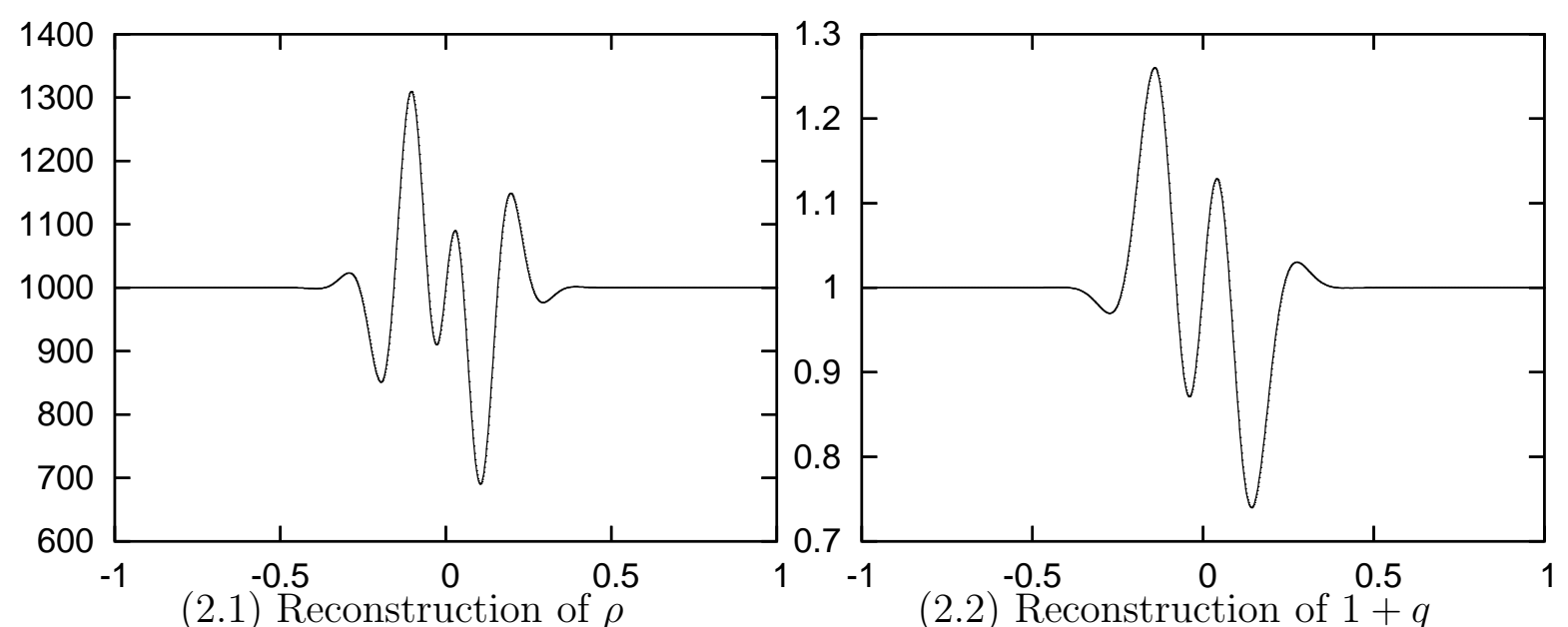

(2.2) Reconstruction of $1+q$
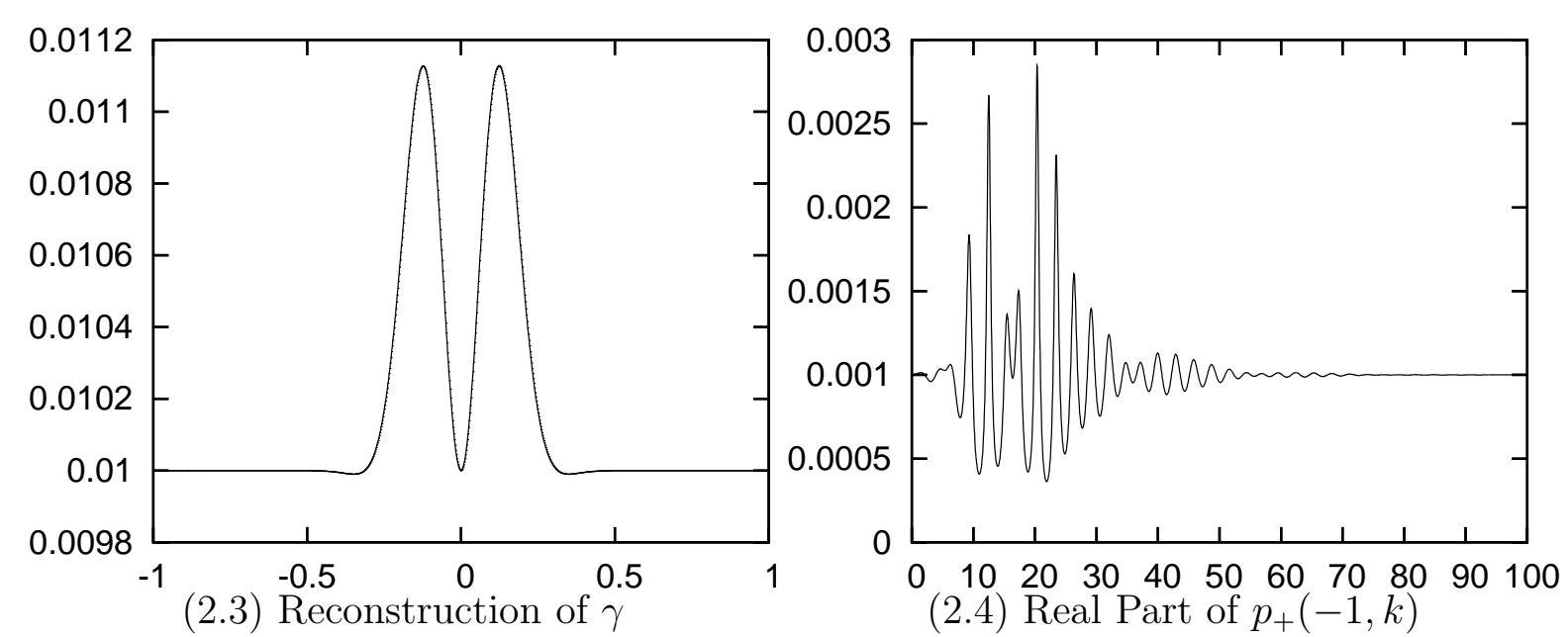

Figure 2: Reconstruction of Example 2 with $a=100$

This is another example of a smooth scatterer in the interval of $[-1,1]$, i.e., $\rho, q, \gamma, \in$ $C_{0}^{\infty}[-1,1]$. Tables 3 and 4 illustrate the numerical behavior of the algorithm; graphs containing the exact and the reconstructed $\rho, q, \gamma$ as well as the input impedance functions $p_{+}(-1, k)$ are included in Figure 2. The algorithm also yields superalgebraic convergence, although not as fast as that of Example 1 due to the highly oscillating character of the scatterer and the impedance functions, as can be seen in Figure 2 . 
Table 1: CPU time and accuracies for Example 1

\begin{tabular}{|c|c|c|c|c|c|c|}
\hline$a$ & $h_{k}$ & $N_{x}$ & $E_{\rho}^{2}$ & $E_{q}^{2}$ & $E_{\gamma}^{2}$ & $t_{C P U}$ \\
\hline 25 & 0.2 & 250 & $3.19 \mathrm{E}-05$ & $6.95 \mathrm{E}-05$ & $4.45 \mathrm{E}-05$ & $3.5 \mathrm{E}+00$ \\
50 & 0.2 & 250 & $9.96 \mathrm{E}-06$ & $2.05 \mathrm{E}-05$ & $1.13 \mathrm{E}-05$ & $7.0 \mathrm{E}+00$ \\
50 & 0.1 & 500 & $7.20 \mathrm{E}-09$ & $1.48 \mathrm{E}-08$ & $8.73 \mathrm{E}-09$ & $1.9 \mathrm{E}+01$ \\
50 & 0.05 & 1000 & $7.13 \mathrm{E}-09$ & $1.47 \mathrm{E}-08$ & $8.60 \mathrm{E}-09$ & $5.8 \mathrm{E}+01$ \\
100 & 0.2 & 500 & $9.54 \mathrm{E}-10$ & $2.00 \mathrm{E}-09$ & $1.28 \mathrm{E}-09$ & $1.8 \mathrm{E}+01$ \\
100 & 0.1 & 1000 & $1.56 \mathrm{E}-12$ & $3.22 \mathrm{E}-12$ & $3.23 \mathrm{E}-12$ & $5.9 \mathrm{E}+01$ \\
100 & 0.05 & 2000 & $4.21 \mathrm{E}-12$ & $9.08 \mathrm{E}-12$ & $9.08 \mathrm{E}-12$ & $2.3 \mathrm{E}+02$ \\
\hline
\end{tabular}

Table 2: CPU time and accuracies for Example 1

\begin{tabular}{|c|c|c|c|c|c|c|}
\hline$a$ & $h_{k}$ & $N_{x}$ & $E_{\rho}^{\infty}$ & $E_{q}^{\infty}$ & $E_{\gamma}^{\infty}$ & $t_{C P U}$ \\
\hline 25 & 0.2 & 250 & $9.48 \mathrm{E}-05$ & $1.83 \mathrm{E}-04$ & $1.26 \mathrm{E}-04$ & $3.5 \mathrm{E}+00$ \\
50 & 0.2 & 250 & $3.03 \mathrm{E}-05$ & $5.56 \mathrm{E}-05$ & $3.90 \mathrm{E}-05$ & $7.0 \mathrm{E}+00$ \\
50 & 0.1 & 500 & $1.87 \mathrm{E}-08$ & $3.50 \mathrm{E}-08$ & $2.05 \mathrm{E}-08$ & $1.9 \mathrm{E}+01$ \\
50 & 0.05 & 1000 & $1.87 \mathrm{E}-08$ & $3.50 \mathrm{E}-08$ & $2.03 \mathrm{E}-08$ & $5.8 \mathrm{E}+01$ \\
100 & 0.2 & 500 & $3.46 \mathrm{E}-09$ & $6.43 \mathrm{E}-09$ & $4.52 \mathrm{E}-09$ & $1.8 \mathrm{E}+01$ \\
100 & 0.1 & 1000 & $3.74 \mathrm{E}-12$ & $7.28 \mathrm{E}-12$ & $7.28 \mathrm{E}-12$ & $5.9 \mathrm{E}+01$ \\
100 & 0.05 & 2000 & $9.58 \mathrm{E}-12$ & $1.82 \mathrm{E}-11$ & $1.82 \mathrm{E}-11$ & $2.3 \mathrm{E}+02$ \\
\hline
\end{tabular}

Table 3: CPU time and accuracies for Example 2

\begin{tabular}{|c|c|c|c|c|c|c|}
\hline$a$ & $h_{k}$ & $N_{x}$ & $E_{\rho}^{2}$ & $E_{q}^{2}$ & $E_{\gamma}^{2}$ & $t_{C P U}$ \\
\hline 50 & 0.05 & 1000 & $1.08 \mathrm{E}-02$ & $2.68 \mathrm{E}-02$ & $2.66 \mathrm{E}-03$ & $5.9 \mathrm{E}+01$ \\
100 & 0.1 & 1000 & $5.66 \mathrm{E}-04$ & $2.25 \mathrm{E}-03$ & $2.04 \mathrm{E}-03$ & $6.0 \mathrm{E}+01$ \\
100 & 0.05 & 1000 & $3.79 \mathrm{E}-05$ & $9.86 \mathrm{E}-05$ & $3.70 \mathrm{E}-05$ & $1.4 \mathrm{E}+02$ \\
100 & 0.025 & 2000 & $3.91 \mathrm{E}-05$ & $1.05 \mathrm{E}-04$ & $4.02 \mathrm{E}-05$ & $5.0 \mathrm{E}+02$ \\
200 & 0.1 & 2000 & $3.65 \mathrm{E}-04$ & $1.45 \mathrm{E}-03$ & $1.32 \mathrm{E}-03$ & $2.3 \mathrm{E}+02$ \\
200 & 0.05 & 2000 & $3.32 \mathrm{E}-08$ & $1.28 \mathrm{E}-07$ & $8.83 \mathrm{E}-08$ & $5.0 \mathrm{E}+02$ \\
200 & 0.05 & 4000 & $3.14 \mathrm{E}-08$ & $1.21 \mathrm{E}-07$ & $8.08 \mathrm{E}-08$ & $9.0 \mathrm{E}+02$ \\
\hline
\end{tabular}

Table 4: CPU time and accuracies for Example 2

\begin{tabular}{|c|c|c|c|c|c|c|}
\hline$a$ & $h_{k}$ & $N_{x}$ & $E_{\rho}^{\infty}$ & $E_{q}^{\infty}$ & $E_{\gamma}^{\infty}$ & $t_{C P U}$ \\
\hline 50 & 0.05 & 1000 & $2.30 \mathrm{E}-02$ & $1.03 \mathrm{E}-01$ & $1.22 \mathrm{E}-02$ & $5.9 \mathrm{E}+01$ \\
100 & 0.1 & 1000 & $1.62 \mathrm{E}-03$ & $1.05 \mathrm{E}-02$ & $8.04 \mathrm{E}-03$ & $6.0 \mathrm{E}+01$ \\
100 & 0.05 & 1000 & $9.99 \mathrm{E}-05$ & $4.14 \mathrm{E}-04$ & $1.57 \mathrm{E}-04$ & $1.4 \mathrm{E}+02$ \\
100 & 0.025 & 2000 & $1.06 \mathrm{E}-04$ & $4.55 \mathrm{E}-04$ & $1.71 \mathrm{E}-04$ & $5.0 \mathrm{E}+02$ \\
200 & 0.1 & 2000 & $1.04 \mathrm{E}-03$ & $6.83 \mathrm{E}-03$ & $5.23 \mathrm{E}-03$ & $2.3 \mathrm{E}+02$ \\
200 & 0.05 & 2000 & $9.83 \mathrm{E}-08$ & $5.99 \mathrm{E}-07$ & $3.55 \mathrm{E}-07$ & $5.0 \mathrm{E}+02$ \\
200 & 0.05 & 4000 & $9.28 \mathrm{E}-08$ & $5.62 \mathrm{E}-07$ & $3.26 \mathrm{E}-07$ & $9.0 \mathrm{E}+02$ \\
\hline
\end{tabular}


Example 3 : In this example, we reconstruct a scatterer defined by the formulae

$$
\begin{gathered}
t=(x+1) \cdot \pi \\
\rho(x)=1000+100 \cdot\left((1-\cos (4 t))-\frac{22}{25}(1-\cos (5 t))+\frac{6}{49}(1-\cos (7 t))\right), \\
q(x)=0.4 \cdot\left((1-\cos (3 t))-\frac{1215}{2783}(1-\cos (11 t))+\frac{7}{23}(1-\cos (12 t))\right), \\
\gamma(x)=0.01+0.003 \cdot\left((1-\cos (2 t))-\frac{16}{21}(1-\cos (3 t))+\frac{5}{28}(1-\cos (4 t))\right) .
\end{gathered}
$$

The scatterer is a $c_{0}^{5}$-function in $R$ with support in the interval $[-1,1]$. The performance of the algorithm is demonstrated in Tables 5, 6 and Figures 3, 4. As we can see from those tables, the convergence of the algorithm is actually better than our prediction of $4^{\text {th }}$-order convergence. 

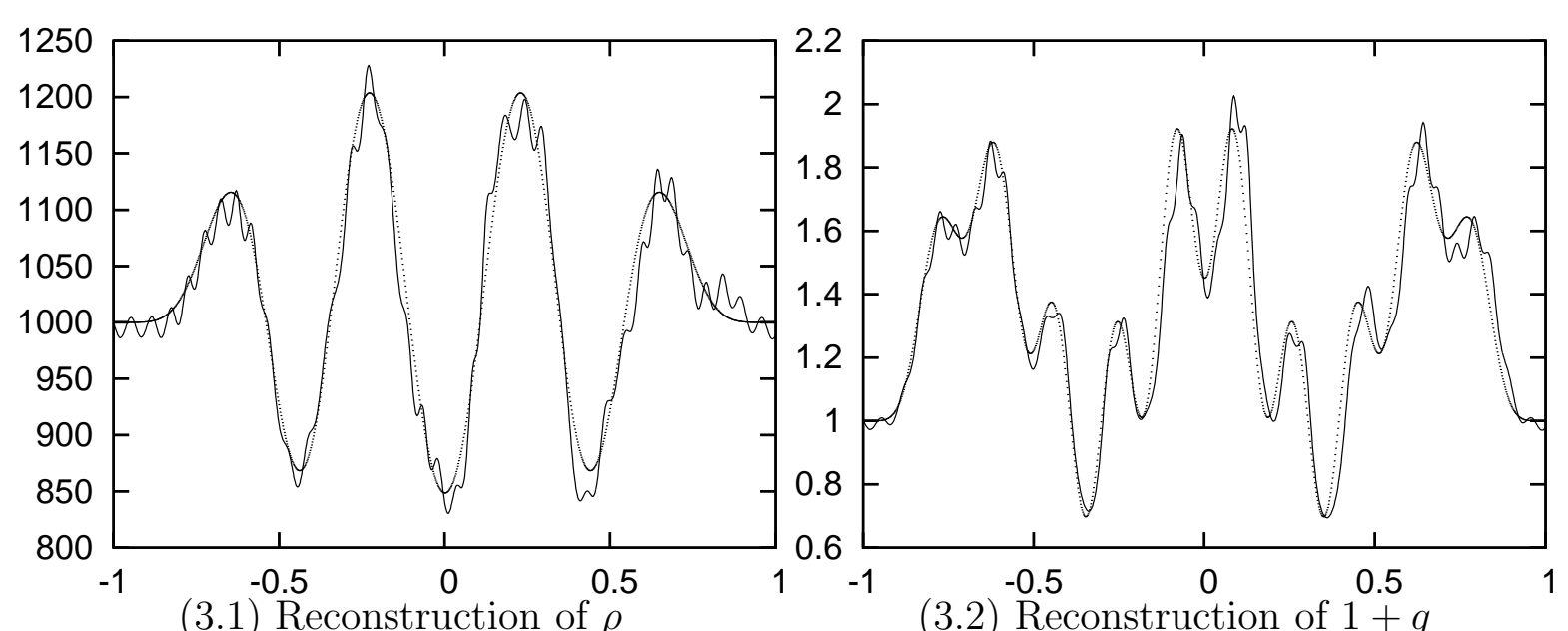

(3.2) Reconstruction of $1+q$

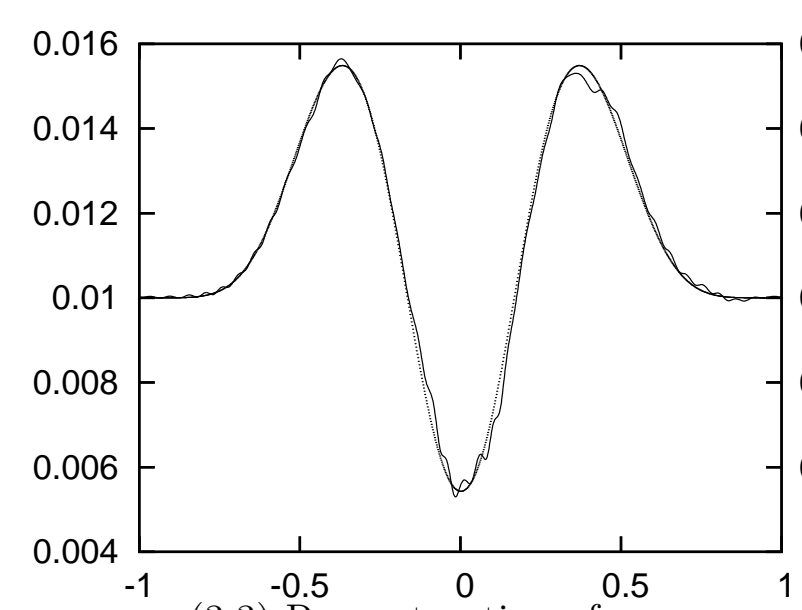

(3.3) Reconstruction of $\gamma$

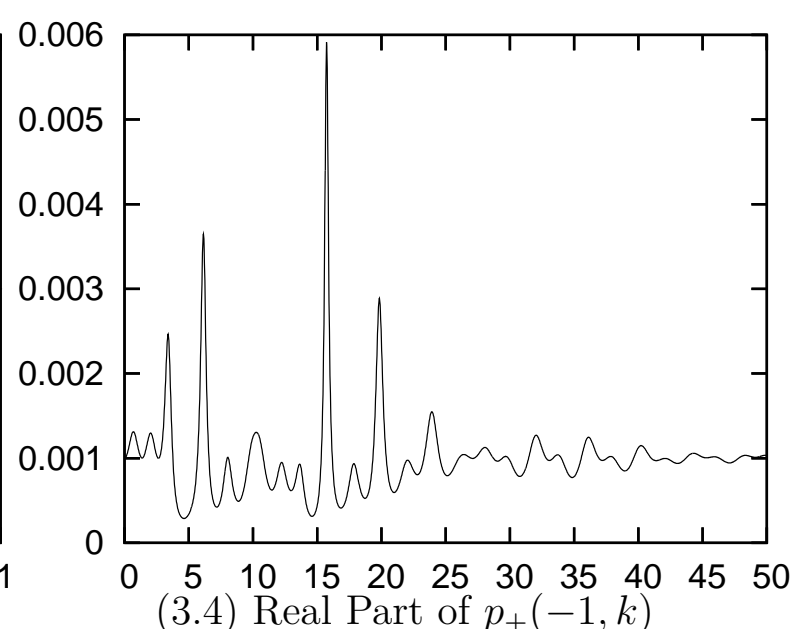

Figure 3: Reconstruction of Example 3 with $a=50$ 


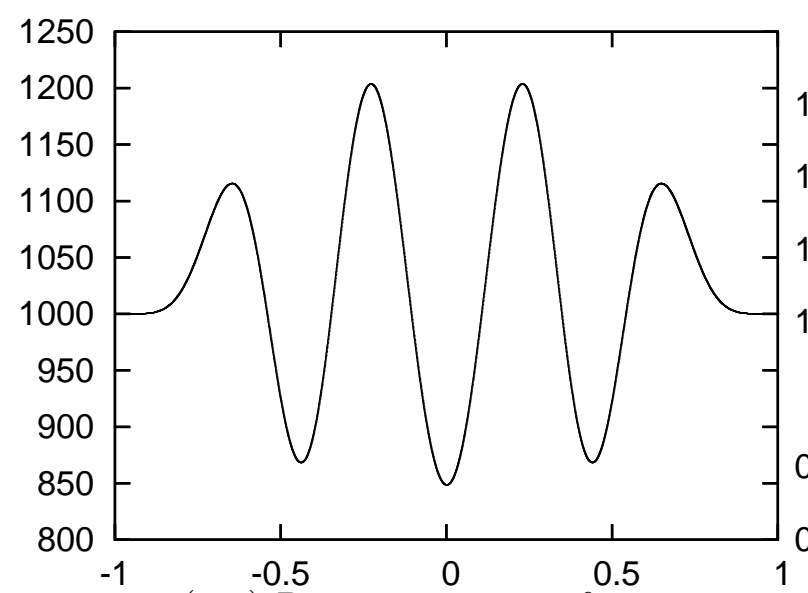

(4.1) Reconstruction of $\rho$

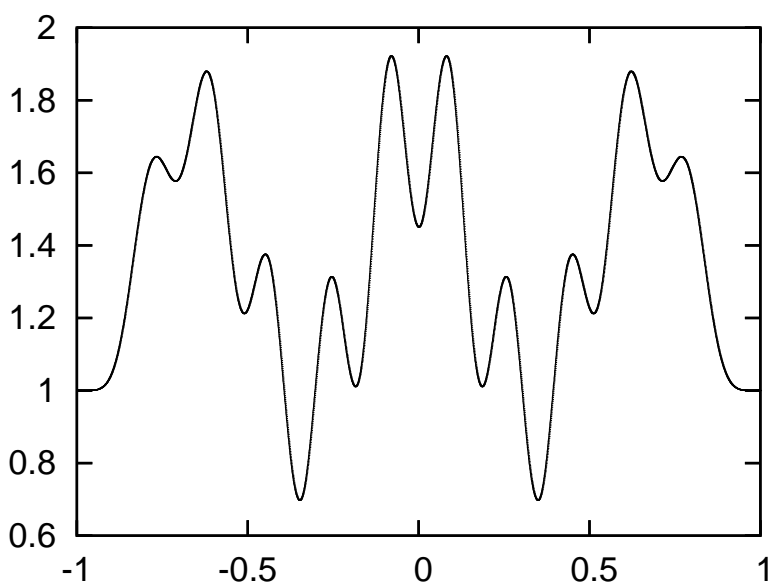

(4.2) Reconstruction of $1+q$

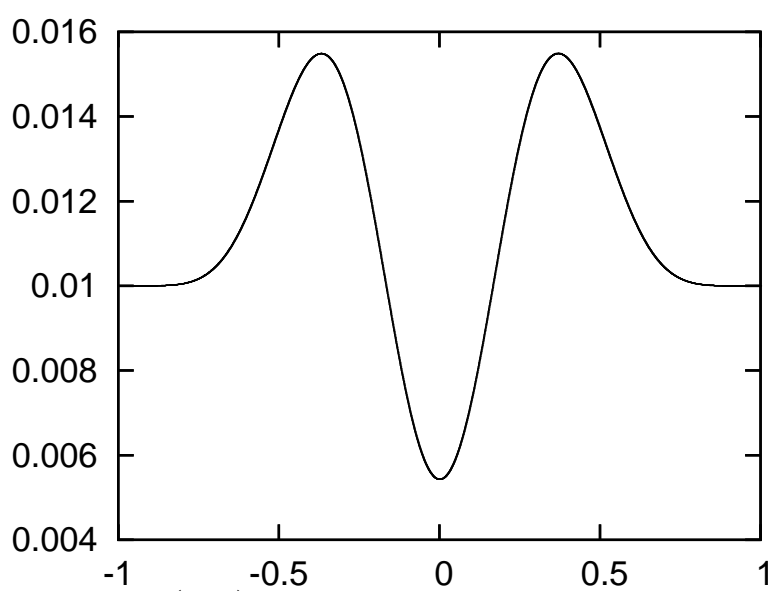

(4.3) Reconstruction of $\gamma$

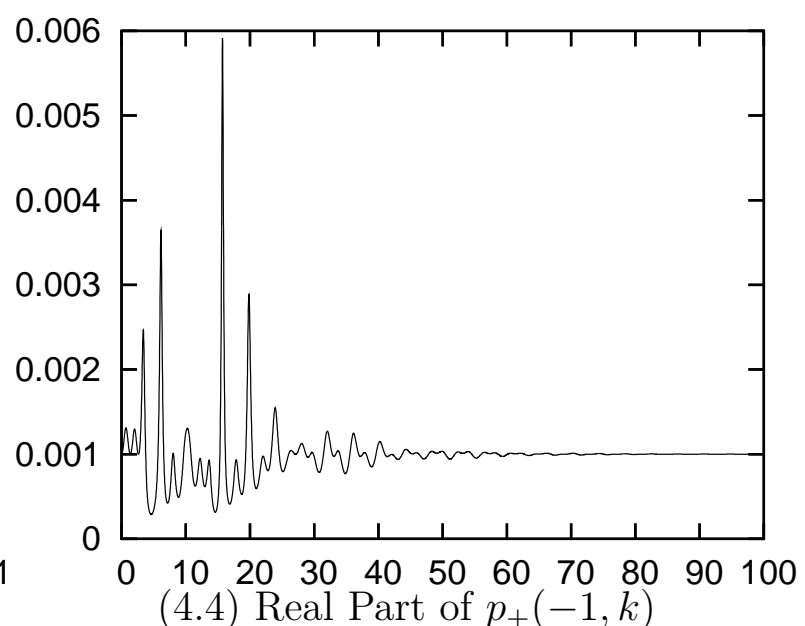

(4.4) Real Part of $p_{+}(-1, k)$

Figure 4: Reconstruction of Example 3 with $a=100$ 
Table 5: CPU time and accuracies for Example 3

\begin{tabular}{|c|c|c|c|c|c|c|}
\hline$a$ & $h_{k}$ & $N_{x}$ & $E_{\rho}^{2}$ & $E_{q}^{2}$ & $E_{\gamma}^{2}$ & $t_{C P U}$ \\
\hline 25 & 0.025 & 1000 & $7.95 \mathrm{E}-02$ & $2.58 \mathrm{E}-01$ & $6.78 \mathrm{E}-02$ & $1.7 \mathrm{E}+01$ \\
50 & 0.025 & 1000 & $1.86 \mathrm{E}-02$ & $6.57 \mathrm{E}-02$ & $1.84 \mathrm{E}-02$ & $4.1 \mathrm{E}+01$ \\
100 & 0.025 & 4000 & $1.87 \mathrm{E}-04$ & $4.72 \mathrm{E}-04$ & $3.34 \mathrm{E}-04$ & $3.5 \mathrm{E}+02$ \\
200 & 0.0125 & 8000 & $8.50 \mathrm{E}-07$ & $2.55 \mathrm{E}-06$ & $9.45 \mathrm{E}-07$ & $2.7 \mathrm{E}+03$ \\
\hline
\end{tabular}

Table 6: CPU time and accuracies for Example 3

\begin{tabular}{|c|c|c|c|c|c|c|}
\hline$a$ & $h_{k}$ & $N_{x}$ & $E_{\rho}^{\infty}$ & $E_{q}^{\infty}$ & $E_{\gamma}^{\infty}$ & $t_{C P U}$ \\
\hline 25 & 0.025 & 1000 & $2.44 \mathrm{E}-01$ & $1.24 \mathrm{E}+00$ & $4.40 \mathrm{E}-01$ & $1.7 \mathrm{E}+01$ \\
50 & 0.025 & 1000 & $5.24 \mathrm{E}-02$ & $2.20 \mathrm{E}-01$ & $1.05 \mathrm{E}-01$ & $4.1 \mathrm{E}+01$ \\
100 & 0.025 & 4000 & $5.18 \mathrm{E}-04$ & $1.56 \mathrm{E}-03$ & $1.12 \mathrm{E}-03$ & $3.5 \mathrm{E}+02$ \\
200 & 0.0125 & 8000 & $2.12 \mathrm{E}-06$ & $7.46 \mathrm{E}-06$ & $3.86 \mathrm{E}-06$ & $2.7 \mathrm{E}+03$ \\
\hline
\end{tabular}


Example 4 : In this example, we construct a scatterer with discontinuous derivatives supported on $[-1,1]$, defined by the formulae

$$
\begin{gathered}
\rho(x)=1000+500 \cdot \sin (7 x), \\
q(x)=0.2 \cdot \cos (30 x) \cdot \frac{e^{3 x}-e^{-3 x}}{e^{3 x}+e^{-3 x}} \\
\gamma(x)=0.01+0.004 \cdot \cos (20 x) \cdot \frac{e^{11 x}-e^{-11 x}}{e^{11 x}+e^{-11 x}} .
\end{gathered}
$$

Tables 7 and 8 illustrate the numerical behavior of the algorithm; Figure 5 demonstrate the exact and the reconstructed $\rho, q, \gamma$, and the input impedance function $p_{+}(-1, k)$. The algorithm is not convergent in this case, although the input impedance function $p_{+}(-1, k)$ behaves properly. Further investigations (see Figure 6) show that, as we move with the ODE solver towards the right boundary of the scattering structure, both the impedance function $p_{+}(x, k)$ and the integrand $p_{+}(x, k)-p_{-}(x, k)$ in the trace formulae (206) become extremely oscillatory and blow up as $k$ increases. This phenomena is closely related to the interaction between non-smooth behavior of $\rho, q$ and the accumulated effect of attenuation. Example 4.1 and Example 4.2 explore this phenomenon in more detail..

Example 4.1: This example uses the same $\rho$ and $q$ as in Example 4, but with zero attenuation; thus we have

$$
\begin{gathered}
\rho(x)=1000+500 \cdot \sin (7 x), \\
q(x)=0.2 \cdot \cos (30 x) \cdot \frac{e^{3 x}-e^{-3 x}}{e^{3 x}+e^{-3 x}}, \\
\gamma(x)=0 .
\end{gathered}
$$

Table 9 illustrates the numerical behavior of the reconstruction algorithm, and Figure 7 contains graphs of the exact and the recovered $\rho, q$. The algorithm exhibits linear convergence. Unlike Example 4, the integrand $p_{+}(x, k)-p_{-}(x, k)$ in the trace formulae (206), and thus in the ODE system, is not increasing with $k$ as the wave travels through the scattering object (see Figure 8).

Example 4.2: This example uses the same $\rho$ and $q$ as in Example 4 and the same $\gamma$ as in Example 3 ( $c_{0}^{5}$-function); thus we have

$$
\begin{gathered}
t=(x+1) \cdot \pi \\
\rho(x)=1000+500 \cdot \sin (7 x), \\
q(x)=0.2 \cdot \cos (30 x) \cdot \frac{e^{3 x}-e^{-3 x}}{e^{3 x}+e^{-3 x}}, \\
\gamma(x)=0.01+0.003 \cdot\left((1-\cos (2 t))-\frac{16}{21}(1-\cos (3 t))+\frac{5}{28}(1-\cos (4 t))\right) .
\end{gathered}
$$

Tables 10, 11 illustrate the numerical behavior of the reconstruction algorithm.

Example 4.3: This example uses the same $q$ and $\gamma$ as in Example 4, but with a constant $\rho$; thus we have

$$
\rho(x)=1000,
$$


Table 7: CPU time and accuracies for Example 4

\begin{tabular}{|c|c|c|c|c|c|c|}
\hline$a$ & $h_{k}$ & $N_{x}$ & $E_{\rho}^{2}$ & $E_{q}^{2}$ & $E_{\gamma}^{2}$ & $t_{C P U}$ \\
\hline 25 & 0.025 & 1000 & $1.74 \mathrm{E}-02$ & $4.10 \mathrm{E}-02$ & $1.73 \mathrm{E}-01$ & $1.7 \mathrm{E}+01$ \\
50 & 0.025 & 1000 & $3.22 \mathrm{E}-02$ & $1.88 \mathrm{E}-01$ & $1.66 \mathrm{E}-01$ & $4.0 \mathrm{E}+01$ \\
100 & 0.025 & 2000 & $2.11 \mathrm{E}-02$ & $1.30 \mathrm{E}-01$ & $8.29 \mathrm{E}-02$ & $1.7 \mathrm{E}+02$ \\
200 & 0.025 & 4000 & $1.71 \mathrm{E}-02$ & $1.13 \mathrm{E}-01$ & $1.62 \mathrm{E}-01$ & $6.7 \mathrm{E}+02$ \\
\hline
\end{tabular}

Table 8: CPU time and accuracies for Example 4

\begin{tabular}{|c|c|c|c|c|c|c|}
\hline$a$ & $h_{k}$ & $N_{x}$ & $E_{\rho}^{\infty}$ & $E_{q}^{\infty}$ & $E_{\gamma}^{\infty}$ & $t_{C P U}$ \\
\hline 25 & 0.025 & 1000 & $4.97 \mathrm{E}-02$ & $1.04 \mathrm{E}-01$ & $4.89 \mathrm{E}-01$ & $1.7 \mathrm{E}+01$ \\
50 & 0.025 & 1000 & $1.27 \mathrm{E}-01$ & $7.17 \mathrm{E}-01$ & $6.67 \mathrm{E}-01$ & $4.0 \mathrm{E}+01$ \\
100 & 0.025 & 2000 & $7.74 \mathrm{E}-02$ & $4.58 \mathrm{E}-01$ & $3.40 \mathrm{E}-01$ & $1.7 \mathrm{E}+02$ \\
200 & 0.025 & 4000 & $8.94 \mathrm{E}-02$ & $5.44 \mathrm{E}-01$ & $7.49 \mathrm{E}-01$ & $6.7 \mathrm{E}+02$ \\
\hline
\end{tabular}

Table 9: CPU time and accuracies for Example 4.1

\begin{tabular}{|c|c|c|c|c|c|c|c|}
\hline$a$ & $h_{k}$ & $N_{x}$ & $E_{\rho}^{2}$ & $E_{q}^{2}$ & $E_{\rho}^{\infty}$ & $E_{q}^{\infty}$ & $t_{C P U}$ \\
\hline 25 & 0.025 & 1000 & $1.92 \mathrm{E}-02$ & $6.32 \mathrm{E}-02$ & $5.43 \mathrm{E}-02$ & $1.81 \mathrm{E}-01$ & $8.2 \mathrm{E}+00$ \\
50 & 0.025 & 1000 & $3.15 \mathrm{E}-02$ & $1.85 \mathrm{E}-01$ & $1.22 \mathrm{E}-01$ & $6.91 \mathrm{E}-01$ & $1.7 \mathrm{E}+01$ \\
100 & 0.025 & 2000 & $1.54 \mathrm{E}-02$ & $9.59 \mathrm{E}-02$ & $5.87 \mathrm{E}-02$ & $3.20 \mathrm{E}-01$ & $8.0 \mathrm{E}+01$ \\
200 & 0.05 & 2000 & $5.98 \mathrm{E}-03$ & $3.68 \mathrm{E}-02$ & $2.35 \mathrm{E}-02$ & $1.17 \mathrm{E}-01$ & $8.0 \mathrm{E}+01$ \\
400 & 0.05 & 4000 & $3.33 \mathrm{E}-03$ & $2.06 \mathrm{E}-02$ & $1.31 \mathrm{E}-02$ & $6.90 \mathrm{E}-02$ & $3.5 \mathrm{E}+02$ \\
800 & 0.05 & 4000 & $1.97 \mathrm{E}-03$ & $1.22 \mathrm{E}-02$ & $7.70 \mathrm{E}-03$ & $4.15 \mathrm{E}-02$ & $7.0 \mathrm{E}+02$ \\
\hline
\end{tabular}

Table 10: CPU time and accuracies for Example 4.2

\begin{tabular}{|c|c|c|c|c|c|c|}
\hline$a$ & $h_{k}$ & $N_{x}$ & $E_{\rho}^{2}$ & $E_{q}^{2}$ & $E_{\gamma}^{2}$ & $t_{C P U}$ \\
\hline 25 & 0.025 & 1000 & $1.74 \mathrm{E}-02$ & $3.98 \mathrm{E}-02$ & $1.19 \mathrm{E}-02$ & $1.7 \mathrm{E}+01$ \\
50 & 0.025 & 2000 & $3.26 \mathrm{E}-02$ & $1.90 \mathrm{E}-01$ & $3.68 \mathrm{E}-02$ & $8.1 \mathrm{E}+01$ \\
100 & 0.025 & 2000 & $2.34 \mathrm{E}-02$ & $1.43 \mathrm{E}-01$ & $4.01 \mathrm{E}-02$ & $1.7 \mathrm{E}+02$ \\
200 & 0.05 & 4000 & $3.86 \mathrm{E}-02$ & $2.44 \mathrm{E}-01$ & $1.83 \mathrm{E}-01$ & $3.5 \mathrm{E}+02$ \\
\hline
\end{tabular}

Table 11: CPU time and accuracies for Example 4.2

\begin{tabular}{|c|c|c|c|c|c|c|}
\hline$a$ & $h_{k}$ & $N_{x}$ & $E_{\rho}^{\infty}$ & $E_{q}^{\infty}$ & $E_{\gamma}^{\infty}$ & $t_{C P U}$ \\
\hline 25 & 0.025 & 1000 & $4.93 \mathrm{E}-02$ & $1.03 \mathrm{E}-01$ & $5.33 \mathrm{E}-02$ & $1.7 \mathrm{E}+01$ \\
50 & 0.025 & 2000 & $1.28 \mathrm{E}-01$ & $7.25 \mathrm{E}-01$ & $1.01 \mathrm{E}-01$ & $8.1 \mathrm{E}+01$ \\
100 & 0.025 & 2000 & $8.46 \mathrm{E}-02$ & $5.14 \mathrm{E}-01$ & $9.85 \mathrm{E}-02$ & $1.7 \mathrm{E}+02$ \\
200 & 0.05 & 4000 & $2.50 \mathrm{E}-01$ & $1.63 \mathrm{E}+00$ & $9.21 \mathrm{E}-01$ & $3.5 \mathrm{E}+02$ \\
\hline
\end{tabular}




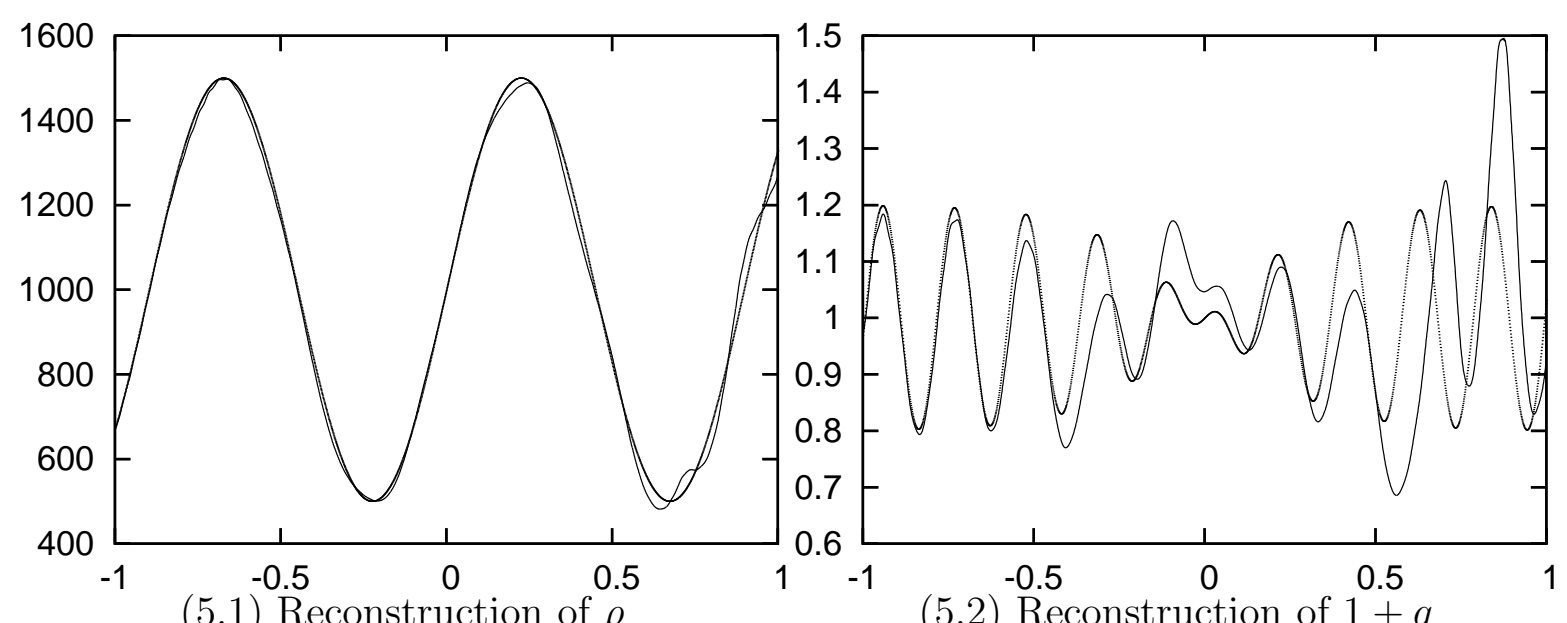

$\begin{array}{ll}\text { (5.1) Reconstruction of } \rho & \text { (5.2) Reconstruction of } 1+q\end{array}$

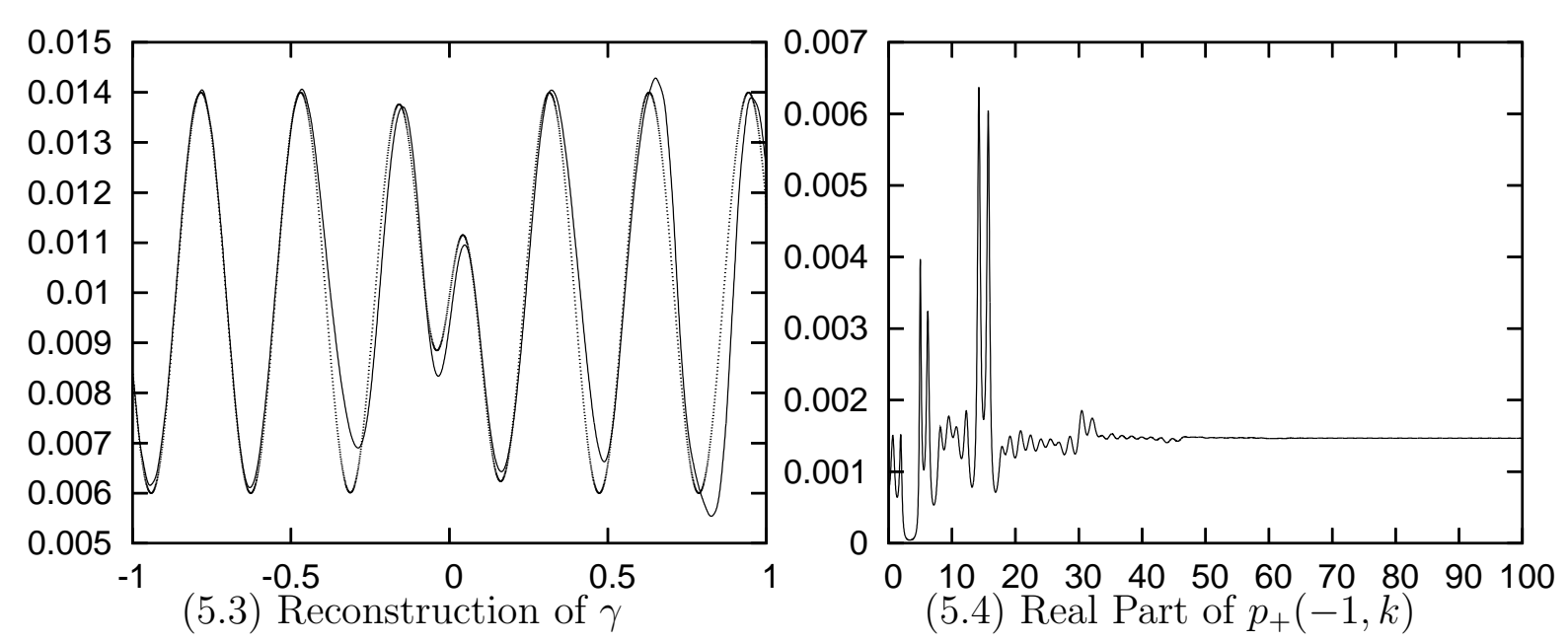

Figure 5: Reconstruction of Example 4 with $a=100$ 

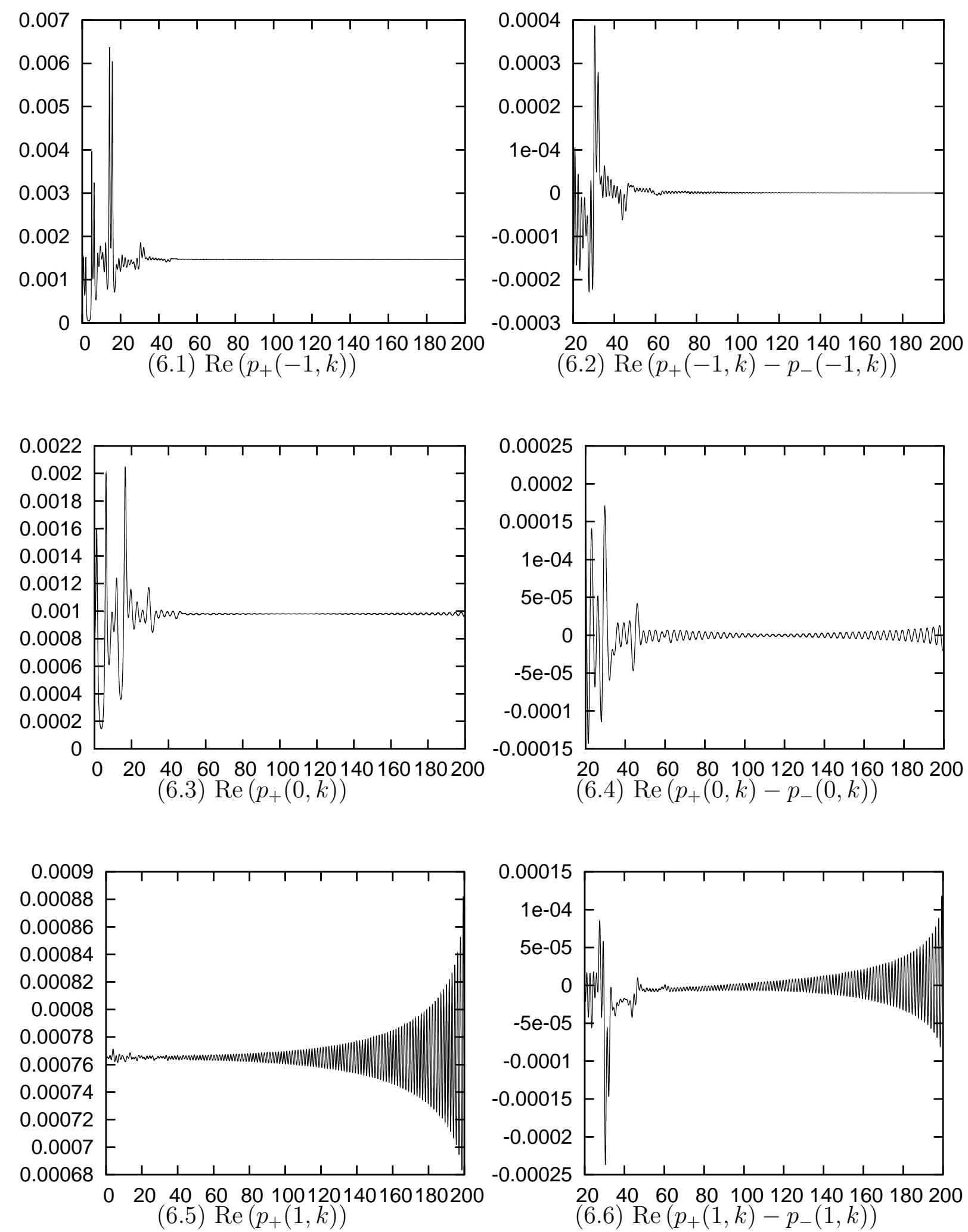

Figure 6: Reconstruction of Example 4 with $a=200$ 

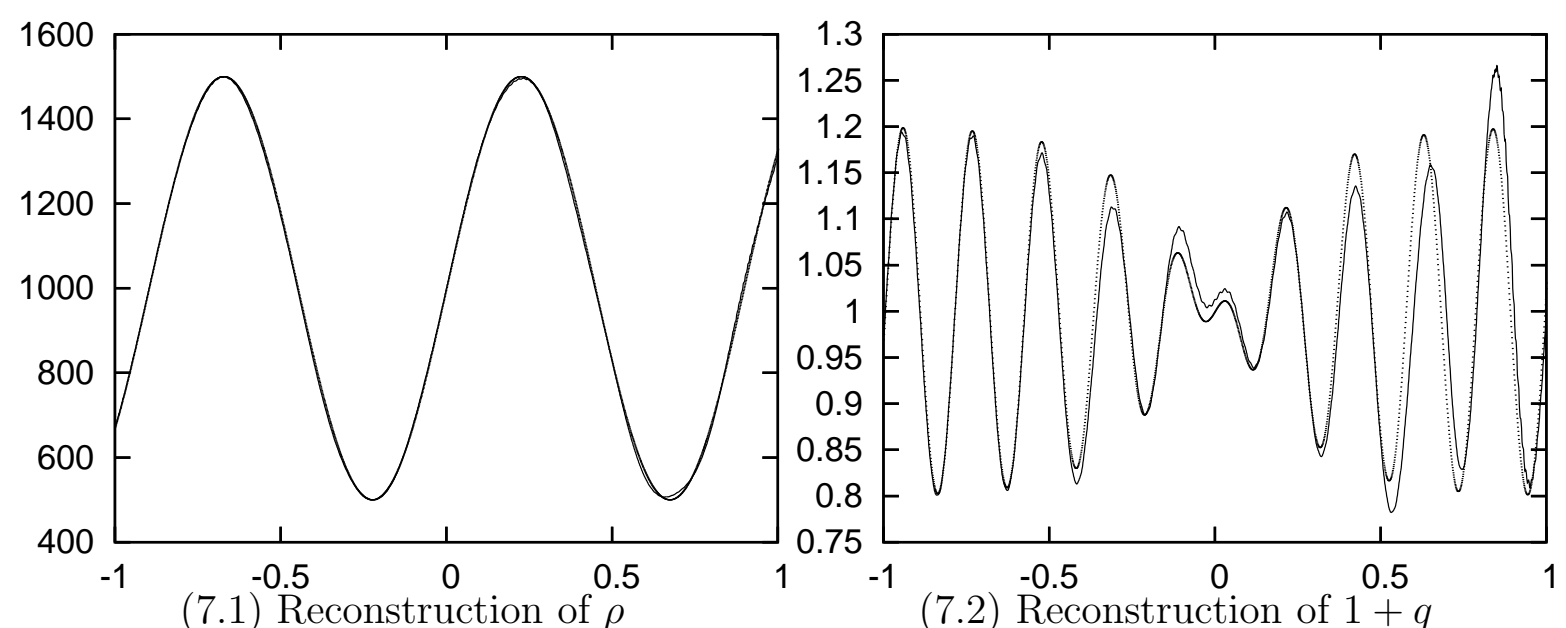

Figure 7: Reconstruction of Example 4.1 with $a=200$

$$
\begin{gathered}
q(x)=0.2 \cdot \cos (30 x) \cdot \frac{e^{3 x}-e^{-3 x}}{e^{3 x}+e^{-3 x}} \\
\gamma(x)=0.01+0.004 \cdot \cos (20 x) \cdot \frac{e^{11 x}-e^{-11 x}}{e^{11 x}+e^{-11 x}} .
\end{gathered}
$$

Table 12 illustrates the numerical behavior of the reconstruction algorithm. Figure 9 contains the exact and the reconstructed $q$ and $\gamma$, and the input impedance function $p_{+}(-1, k)$. Linear convergence is observed for both $q$ and $\gamma$.

Example 4.4: This example uses the same discontinuous $\gamma$ as in Example 4, but with constant $\rho, q$; thus we have

$$
\begin{gathered}
\rho(x)=1000 \\
q(x)=0 \\
\gamma(x)=0.01+0.004 \cdot \cos (20 x) \cdot \frac{e^{11 x}-e^{-11 x}}{e^{11 x}+e^{-11 x}} .
\end{gathered}
$$

Table 13 illustrates the linear convergence of the reconstruction algorithm, as we can expect given Example 4.3. Figure 10 contains the exact and the reconstructed $\gamma$, and the input impedance function $p_{+}(-1, k)$. 

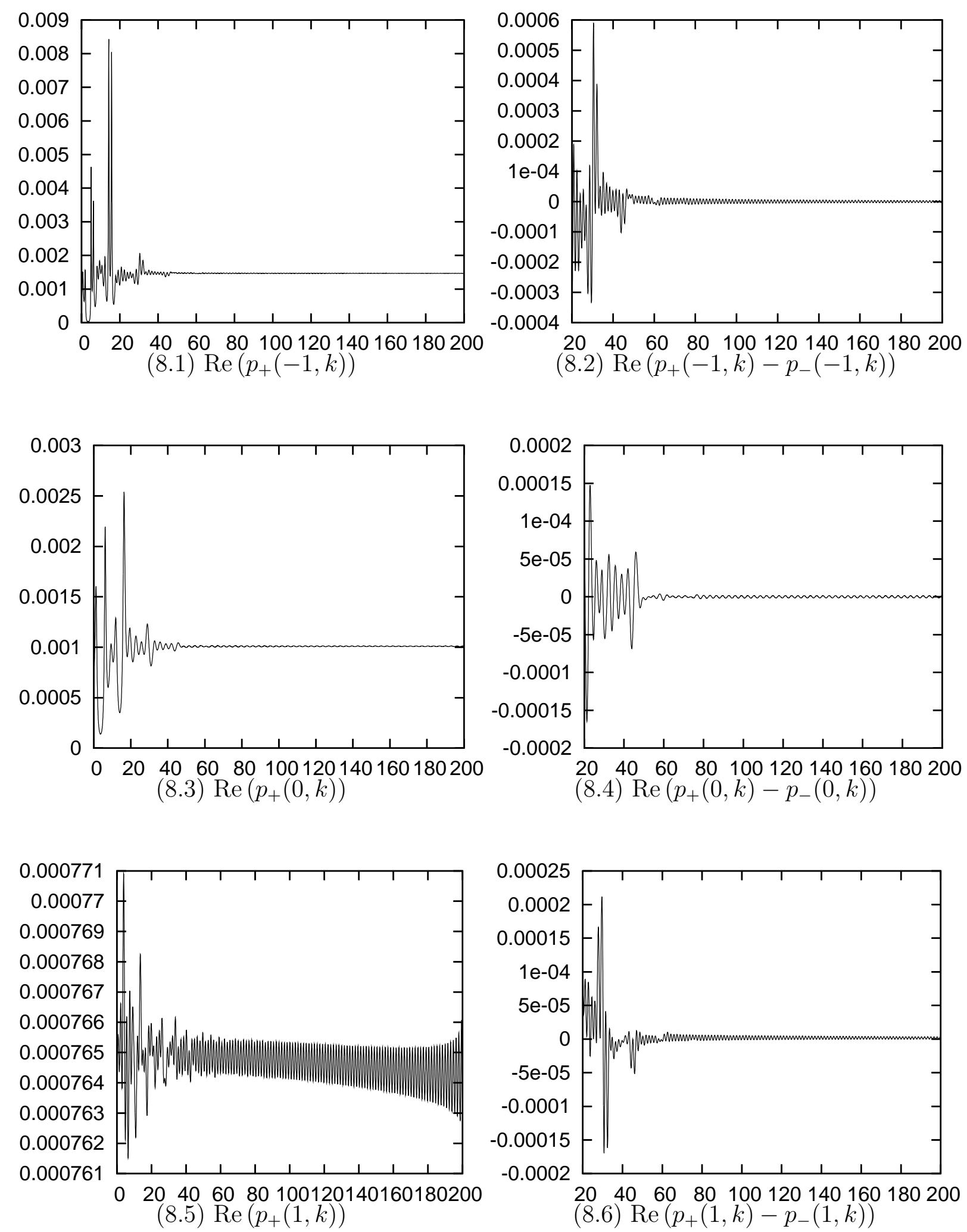

Figure 8: Reconstruction of Example 4.1 with $a=200$ 
Table 12: CPU time and accuracies for Example 4.3

\begin{tabular}{|c|c|c|c|c|c|c|c|}
\hline$a$ & $h_{k}$ & $N_{x}$ & $E_{q}^{2}$ & $E_{\gamma}^{2}$ & $E_{q}^{\infty}$ & $E_{\gamma}^{\infty}$ & $t_{C P U}$ \\
\hline 25 & 0.05 & 1000 & $7.73 \mathrm{E}-02$ & $1.35 \mathrm{E}-01$ & $2.06 \mathrm{E}-01$ & $4.67 \mathrm{E}-01$ & $8.1 \mathrm{E}+00$ \\
50 & 0.1 & 1000 & $4.46 \mathrm{E}-02$ & $1.20 \mathrm{E}-01$ & $1.24 \mathrm{E}-01$ & $4.63 \mathrm{E}-01$ & $8.5 \mathrm{E}+00$ \\
100 & 0.1 & 2000 & $2.22 \mathrm{E}-02$ & $7.67 \mathrm{E}-02$ & $6.61 \mathrm{E}-02$ & $3.41 \mathrm{E}-01$ & $3.3 \mathrm{E}+01$ \\
200 & 0.1 & 4000 & $1.15 \mathrm{E}-02$ & $1.74 \mathrm{E}-02$ & $3.53 \mathrm{E}-02$ & $6.12 \mathrm{E}-02$ & $1.6 \mathrm{E}+02$ \\
\hline
\end{tabular}
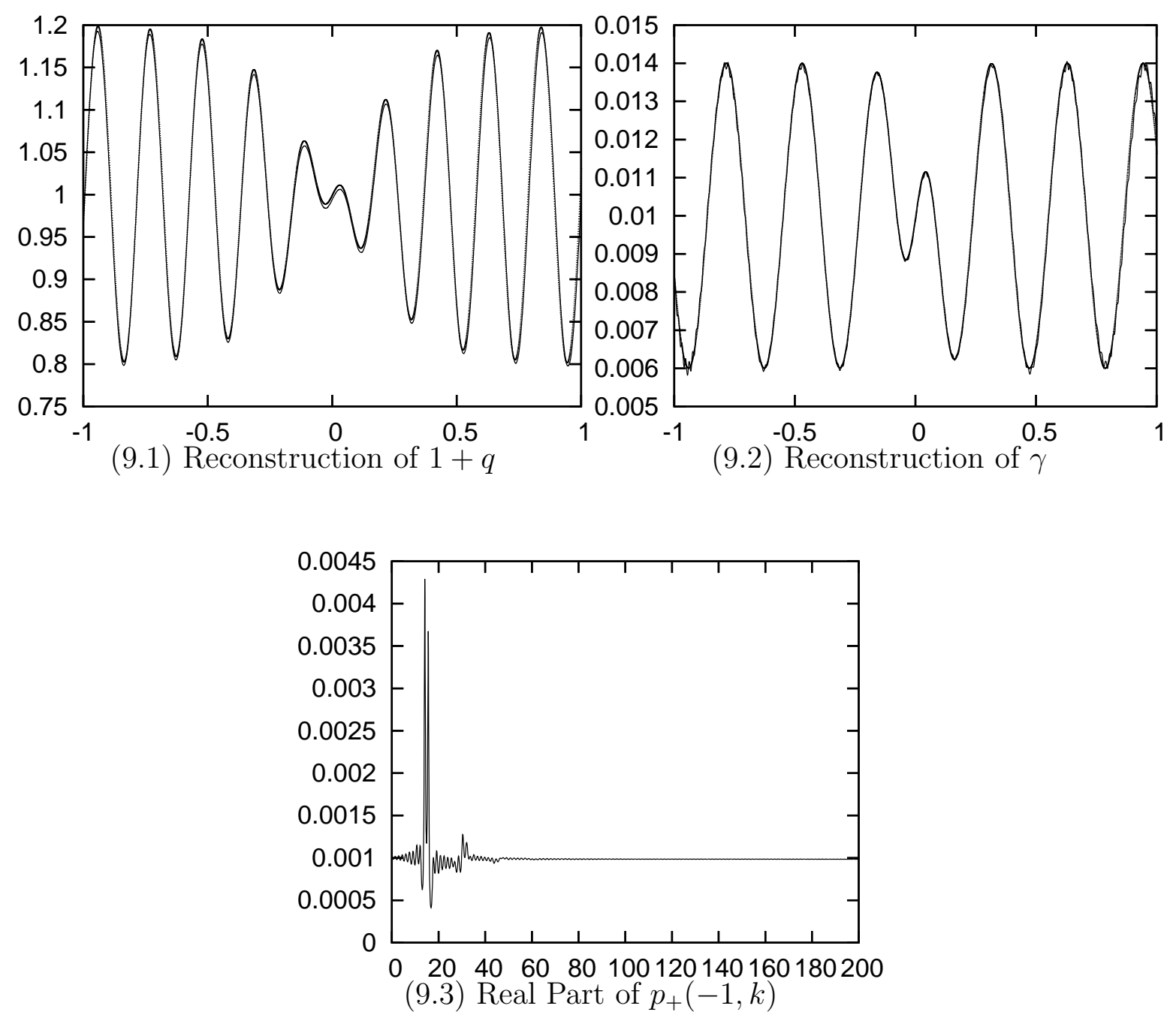

Figure 9: Reconstruction of Example 4.3 with $a=200$ 
Table 13: CPU time and accuracies for Example 4.4

\begin{tabular}{|c|c|c|c|c|c|}
\hline$a$ & $h_{k}$ & $N_{x}$ & $E_{\gamma}^{2}$ & $E_{\gamma}^{\infty}$ & $t_{C P U}$ \\
\hline 25 & 0.05 & 1000 & $4.71 \mathrm{E}-02$ & $1.08 \mathrm{E}-01$ & $8.1 \mathrm{E}+00$ \\
50 & 0.1 & 1000 & $2.26 \mathrm{E}-01$ & $4.51 \mathrm{E}-02$ & $8.0 \mathrm{E}+00$ \\
100 & 0.2 & 2000 & $1.11 \mathrm{E}-02$ & $2.06 \mathrm{E}-02$ & $8.2 \mathrm{E}+01$ \\
200 & 0.2 & 8000 & $5.74 \mathrm{E}-03$ & $1.02 \mathrm{E}-02$ & $6.7 \mathrm{E}+02$ \\
\hline
\end{tabular}

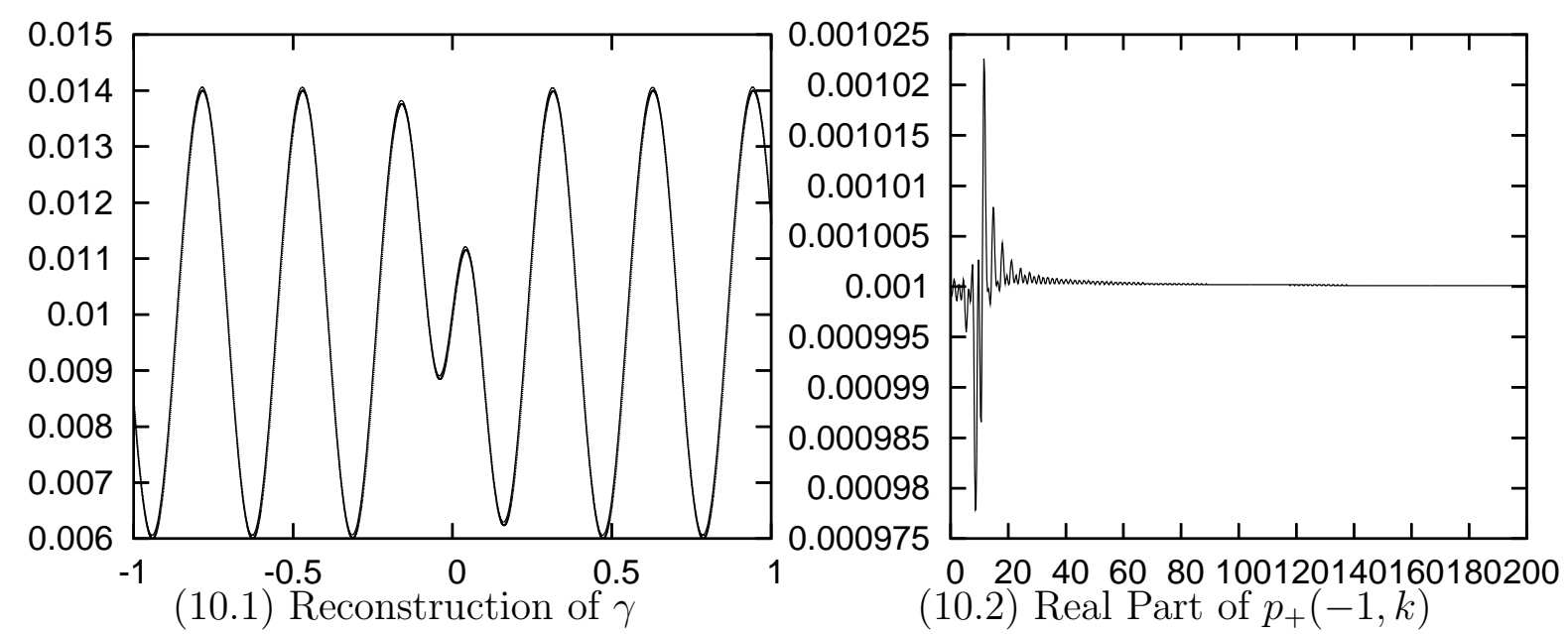

Figure 10: Reconstruction of Example 4.4 with $a=200$ 
Example 5 : In this example, we reconstruct a scatterer defined by the formulae

$$
\begin{gathered}
\rho(x)= \begin{cases}1000 & x \in(-\infty,-0.5] \\
1200 & x \in(-0.5,0.5] \\
1000 & x \in(0.5, \infty)\end{cases} \\
q(x)= \begin{cases}0 & x \in(-\infty,-0.5] \\
0.4 & x \in(-0.5,0.5] \\
0 & x \in(0.5, \infty)\end{cases} \\
\gamma(x)=\left\{\begin{array}{ll}
0.010 & x \in(-\infty,-0.5] \\
0.015 & x \in(-0.5,0.5] \\
0.010 & x \in(0.5, \infty)
\end{array} .\right.
\end{gathered}
$$

In this example, $\rho, q, \gamma$ are discontinuous. Tables 14, 15 illustrates the numerical behavior of the reconstruction algorithm, and Figure 11 contains graphs of the exact and the recovered $\rho, q, \gamma$ and the input impedance function $p_{+}(-1, k)$. The algorithm does not converge as we can expect from Example 4; The integrand $p_{+}(x, k)-p_{-}(x, k)$ is highly oscillatory and blows up as $k$ increase for all $x \in R$ (see Figure 12).

Example 5.1 In this example, we reconstruct a scatterer whose attenuation $\gamma$ is discontinuous and density $\rho$, potential $q$ are constant, given by the formulae

$$
\begin{gathered}
\rho(x)=1000, \\
q(x)=0, \\
\gamma(x)=\left\{\begin{array}{ll}
0.010 & x \in(-\infty,-0.5] \\
0.015 & x \in(-0.5,0.5] \\
0.010 & x \in(0.5, \infty)
\end{array} .\right.
\end{gathered}
$$

The numerical results are demonstrated in Table 16; the exact and the reconstructed $\gamma$, as well as the input impedance function $p_{+}(-1, k)$ are contained in Figure 13. The convergence of the algorithm is of the order $O\left(\frac{1}{\sqrt{a}}\right)$, where $a$ is the largest frequency in the reconstruction algorithm. The convergence of the algorithm for discontinuous $\gamma$ is quite similar to that of discontinuous $\rho$, as reported in [3]. 
Table 14: CPU time and accuracies for Example 5

\begin{tabular}{|c|c|c|c|c|c|c|}
\hline$a$ & $h_{k}$ & $N_{x}$ & $E_{\rho}^{2}$ & $E_{q}^{2}$ & $E_{\gamma}^{2}$ & $t_{C P U}$ \\
\hline 25 & 0.05 & 500 & $1.18 \mathrm{E}-02$ & $2.58 \mathrm{E}-02$ & $3.08 \mathrm{E}-02$ & $4.2 \mathrm{E}+00$ \\
50 & 0.05 & 1000 & $7.94 \mathrm{E}-03$ & $2.02 \mathrm{E}-02$ & $2.27 \mathrm{E}-02$ & $1.7 \mathrm{E}+01$ \\
100 & 0.025 & 4000 & $1.36 \mathrm{E}-02$ & $2.92 \mathrm{E}-02$ & $2.78 \mathrm{E}-02$ & $1.7 \mathrm{E}+02$ \\
200 & 0.025 & 8000 & $1.28 \mathrm{E}-02$ & $2.76 \mathrm{E}-02$ & $2.53 \mathrm{E}-02$ & $1.3 \mathrm{E}+03$ \\
\hline
\end{tabular}

Table 15: CPU time and accuracies for Example 5

\begin{tabular}{|c|c|c|c|c|c|c|}
\hline$a$ & $h_{k}$ & $N_{x}$ & $E_{\rho}^{\infty}$ & $E_{q}^{\infty}$ & $E_{\gamma}^{\infty}$ & $t_{C P U}$ \\
\hline 25 & 0.05 & 500 & $7.75 \mathrm{E}-02$ & $1.91 \mathrm{E}-01$ & $2.39 \mathrm{E}-01$ & $4.2 \mathrm{E}+00$ \\
50 & 0.05 & 1000 & $6.43 \mathrm{E}-02$ & $1.78 \mathrm{E}-01$ & $2.13 \mathrm{E}-01$ & $1.7 \mathrm{E}+01$ \\
100 & 0.025 & 4000 & $1.25 \mathrm{E}-01$ & $3.52 \mathrm{E}-01$ & $4.93 \mathrm{E}-01$ & $1.7 \mathrm{E}+02$ \\
200 & 0.025 & 8000 & $1.14 \mathrm{E}-01$ & $3.44 \mathrm{E}-01$ & $4.77 \mathrm{E}-01$ & $1.3 \mathrm{E}+03$ \\
\hline
\end{tabular}

Table 16: CPU time and accuracies for Example 5.1

\begin{tabular}{|c|c|c|c|c|c|}
\hline$a$ & $h_{k}$ & $N_{x}$ & $E_{\gamma}^{2}$ & $E_{\gamma}^{\infty}$ & $t_{C P U}$ \\
\hline 25 & 0.05 & 1000 & $3.13 \mathrm{E}-02$ & $2.36 \mathrm{E}-01$ & $8.3 \mathrm{E}+00$ \\
50 & 0.1 & 1000 & $2.20 \mathrm{E}-02$ & $2.37 \mathrm{E}-01$ & $8.7 \mathrm{E}+00$ \\
100 & 0.2 & 1000 & $1.56 \mathrm{E}-02$ & $2.35 \mathrm{E}-01$ & $8.5 \mathrm{E}+00$ \\
200 & 0.2 & 4000 & $1.27 \mathrm{E}-02$ & $2.26 \mathrm{E}-01$ & $6.9 \mathrm{E}+01$ \\
\hline
\end{tabular}




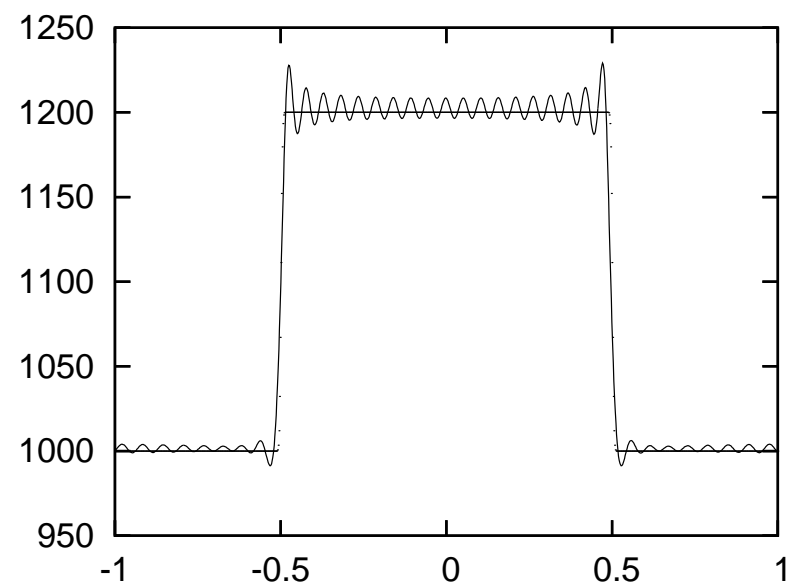

(11.1) Reconstruction of $\rho$

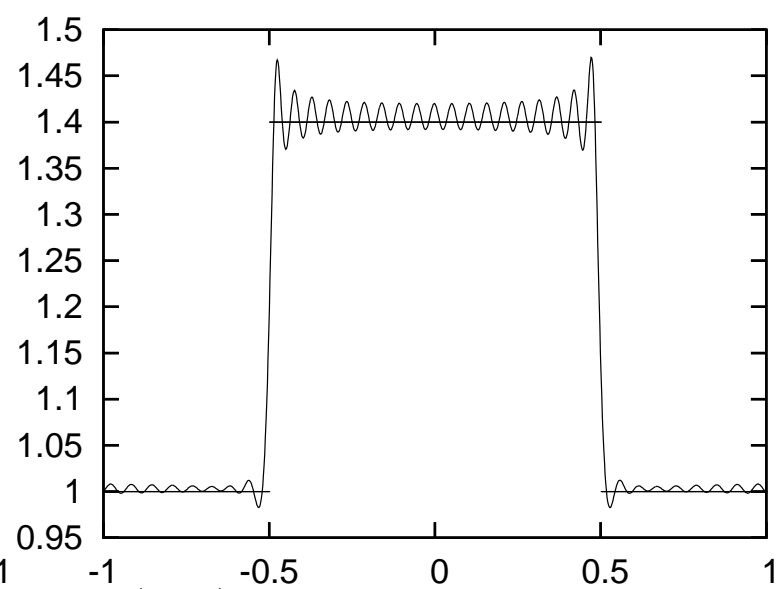

(11.2) Reconstruction of $1+q$

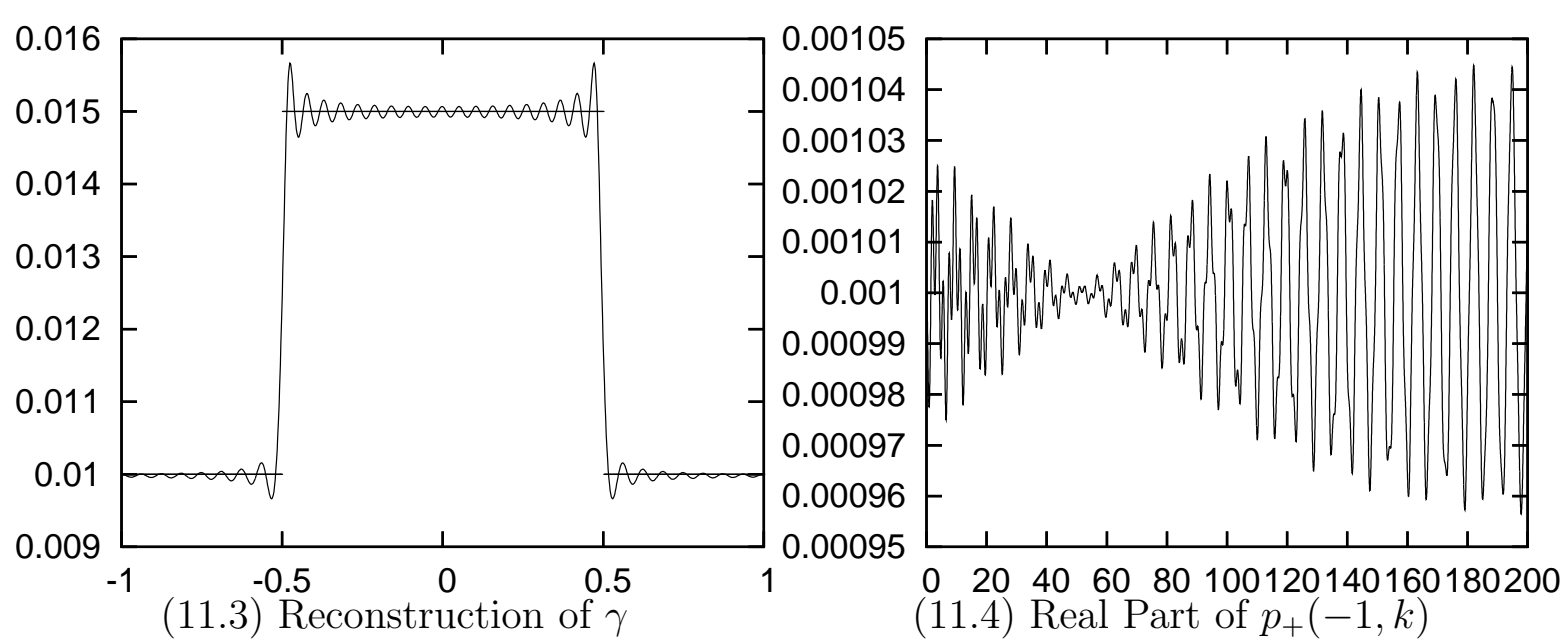

Figure 11: Reconstruction of Example 5 with $a=50$ 


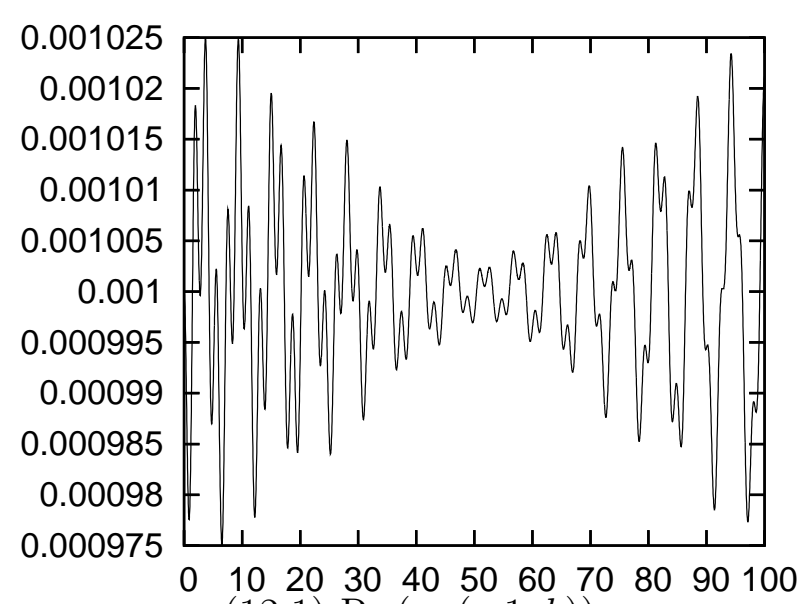

(12.1) $\operatorname{Re}\left(p_{+}(-1, k)\right)$
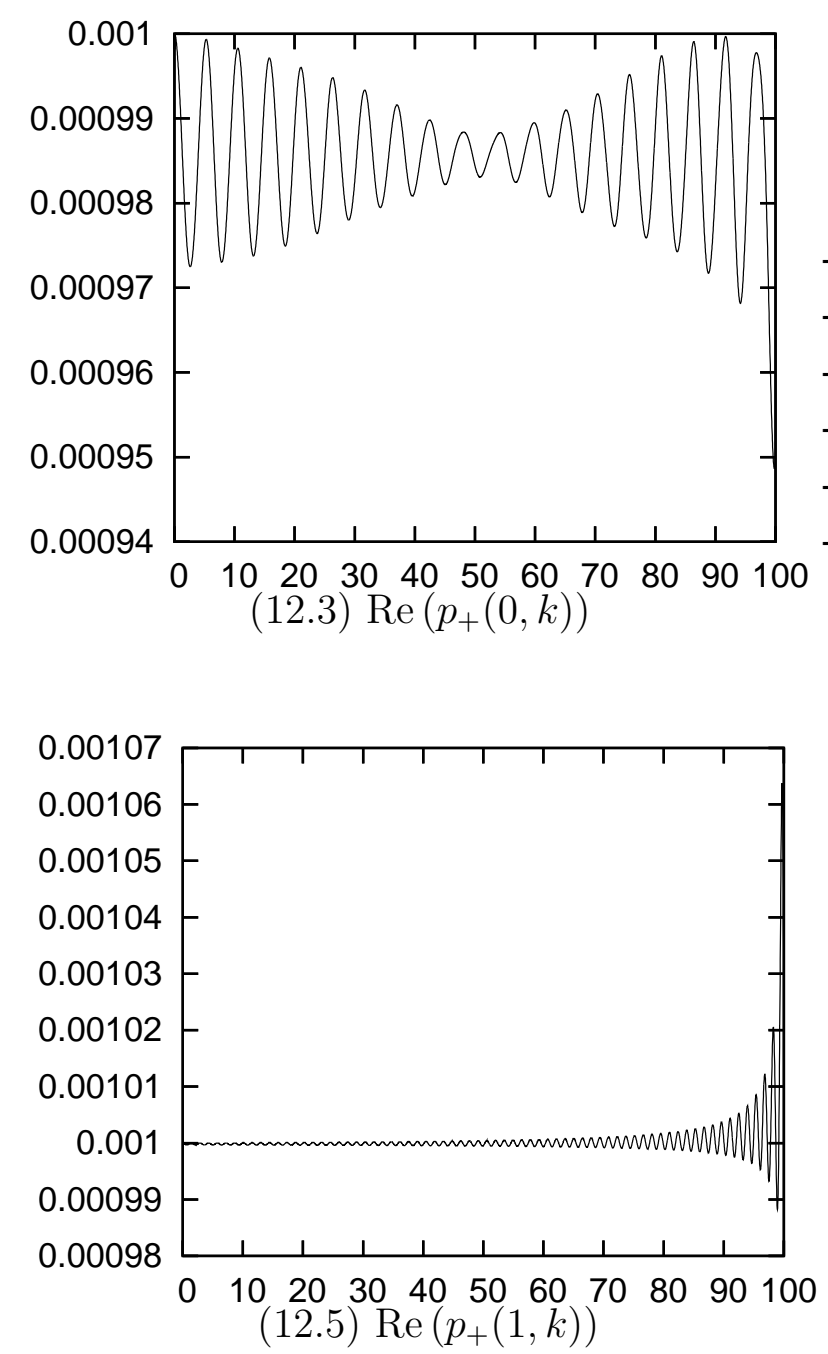

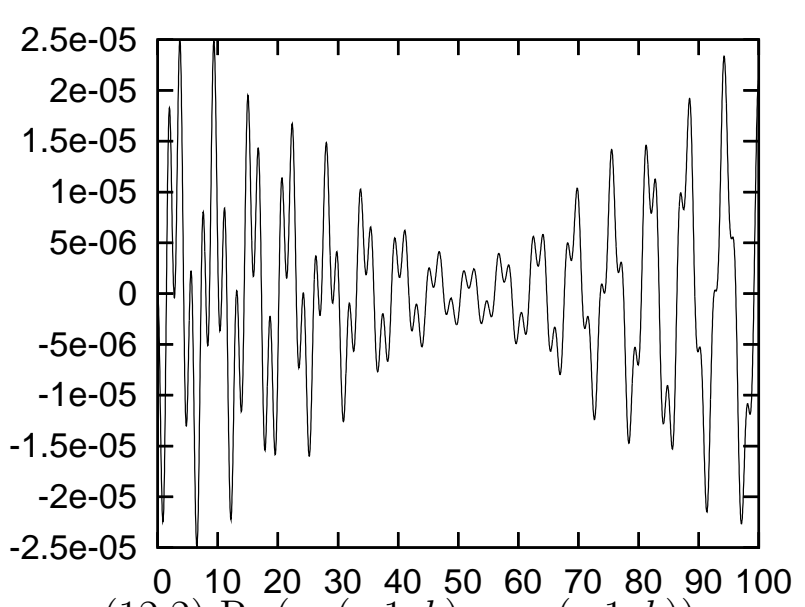

$(12.2) \operatorname{Re}\left(p_{+}(-1, k)-p_{-}(-1, k)\right)$
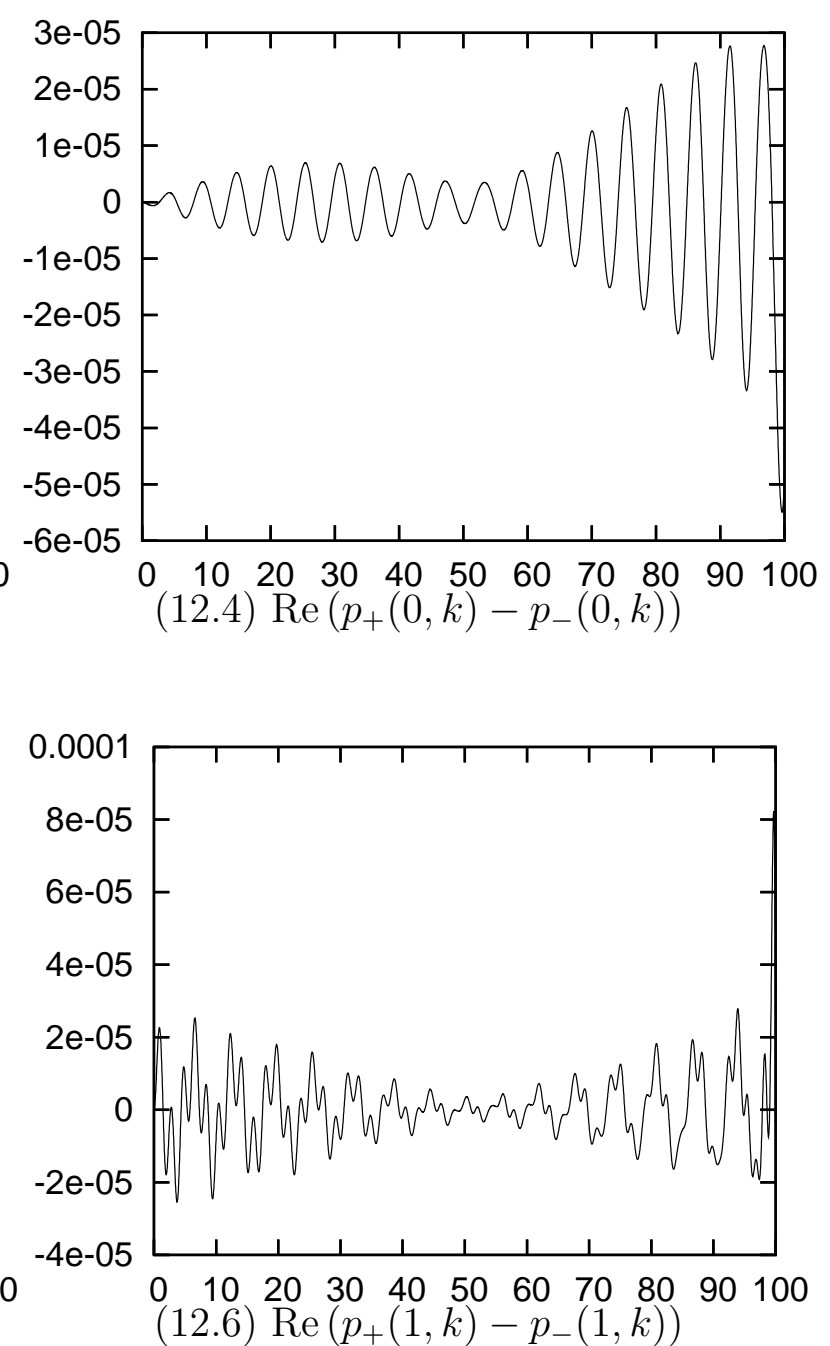

Figure 12: Reconstruction of Example 5 with $a=100$ 


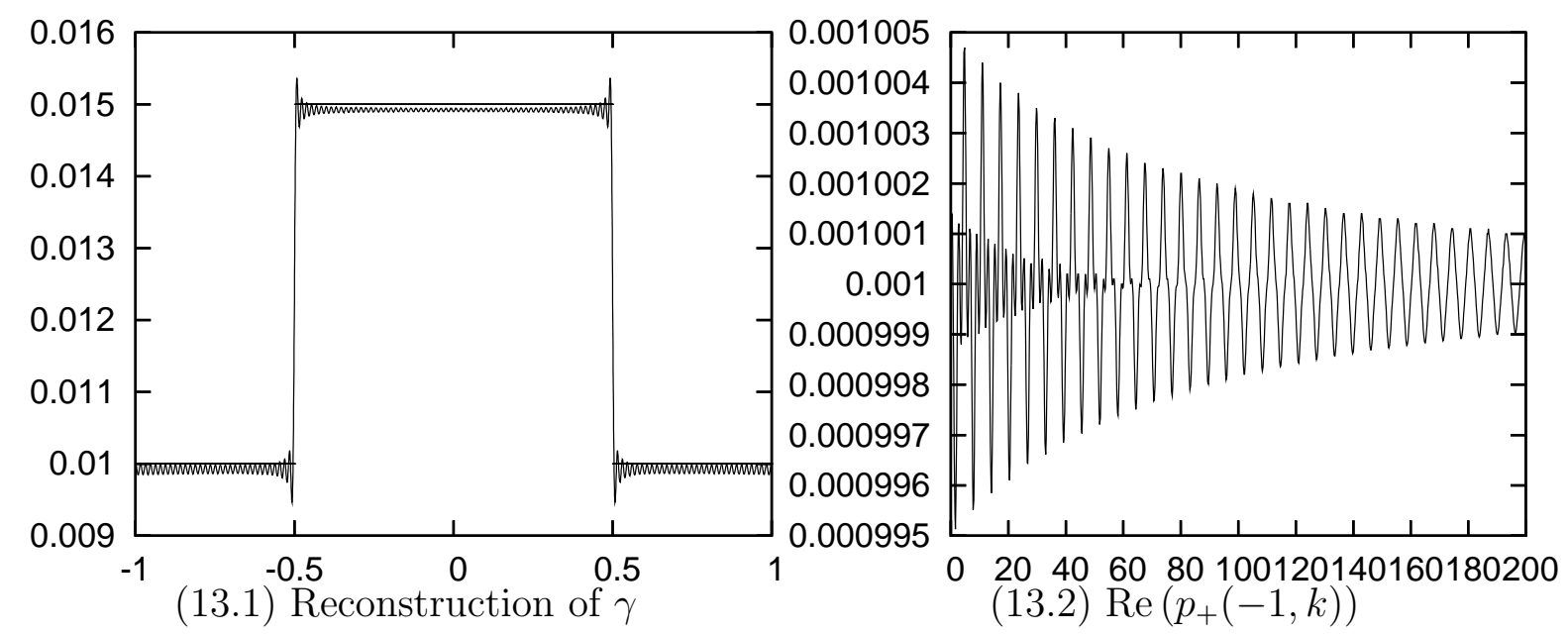

Figure 13: Reconstruction of Example 5.1 with $a=200$ 
Example 6 : Here, we reconstruct a staircase-shaped scatterer defined by the formulae

$$
\begin{gathered}
\rho(x)= \begin{cases}1050 & x \in(-\infty,-0.8] \\
1150 & x \in(-0.8,-0.4] \\
1250 & x \in(-0.4,0.0] \\
1350 & x \in(0.0,0.4] \\
1300 & x \in(0.4,0.8] \\
1200 & x \in(0.8, \infty)\end{cases} \\
q(x)= \begin{cases}0 & x \in(-\infty,-0.8] \\
0.1 & x \in(-0.8,-0.6] \\
0.2 & x \in(-0.6,-0.2] \\
0.3 & x \in(-0.2,0.2] \\
0.2 & x \in(0.2,0.8] \\
0 & x \in(0.8, \infty)\end{cases} \\
\gamma(x)= \begin{cases}0.01 & x \in(-\infty,-0.8] \\
0.012 & x \in(-0.8,-0.6] \\
0.01 & x \in(-0.6,-0.2] \\
0.008 & x \in(-0.2,0.2] \\
0.007 & x \in(0.2,0.6] \\
0.008 & x \in(0.6,0.8] \\
0.009 & x \in(0.8, \infty)\end{cases}
\end{gathered}
$$

The example is similar to the preceding one, but the shape of the scatterer is more complicated. The numerical results are shown in Table 17, 18 and Figures 14, 15, 16, and 17. The algorithm does not converge in this situation. 

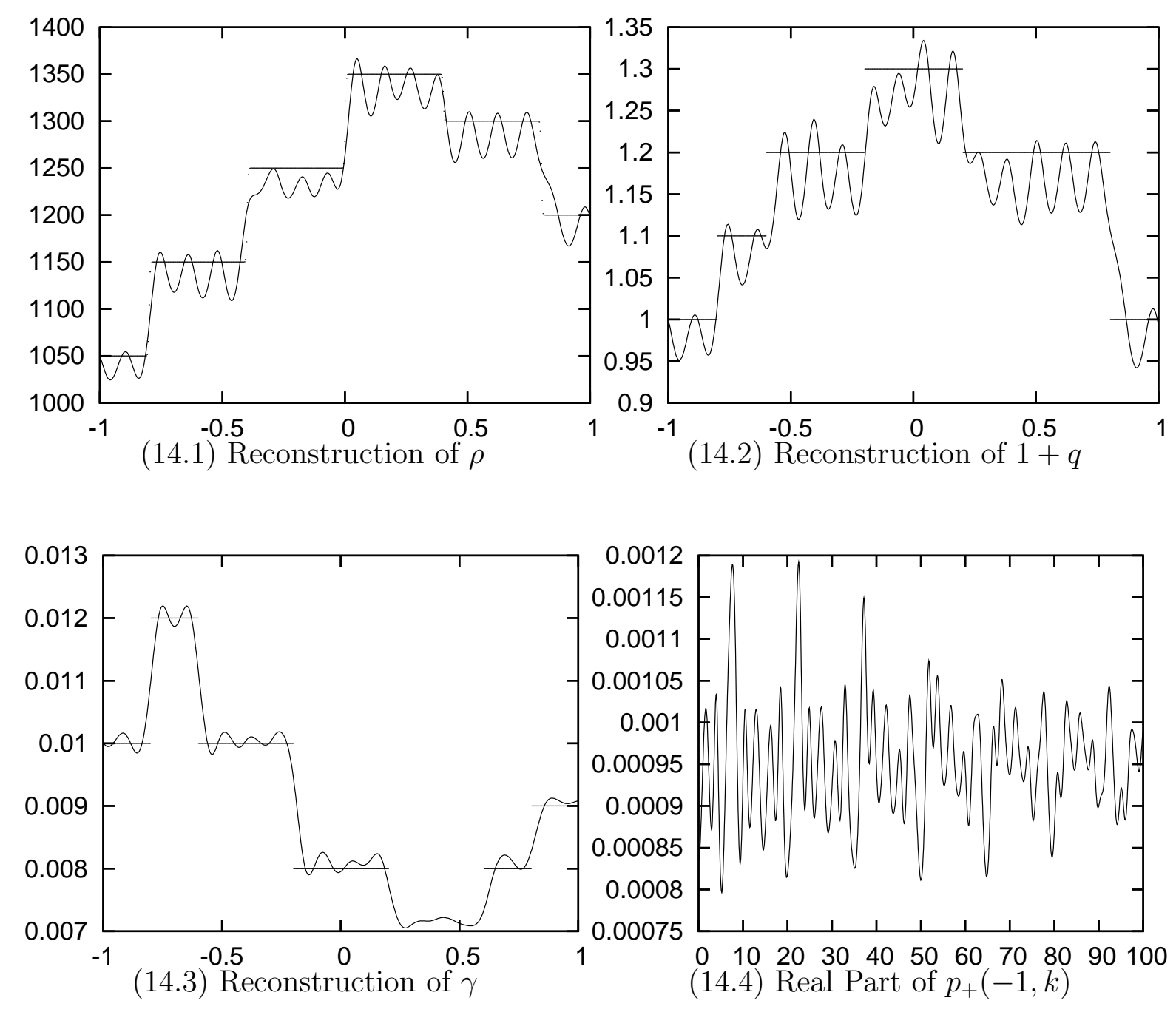

Figure 14: Reconstruction of Example 6 with $a=25$ 

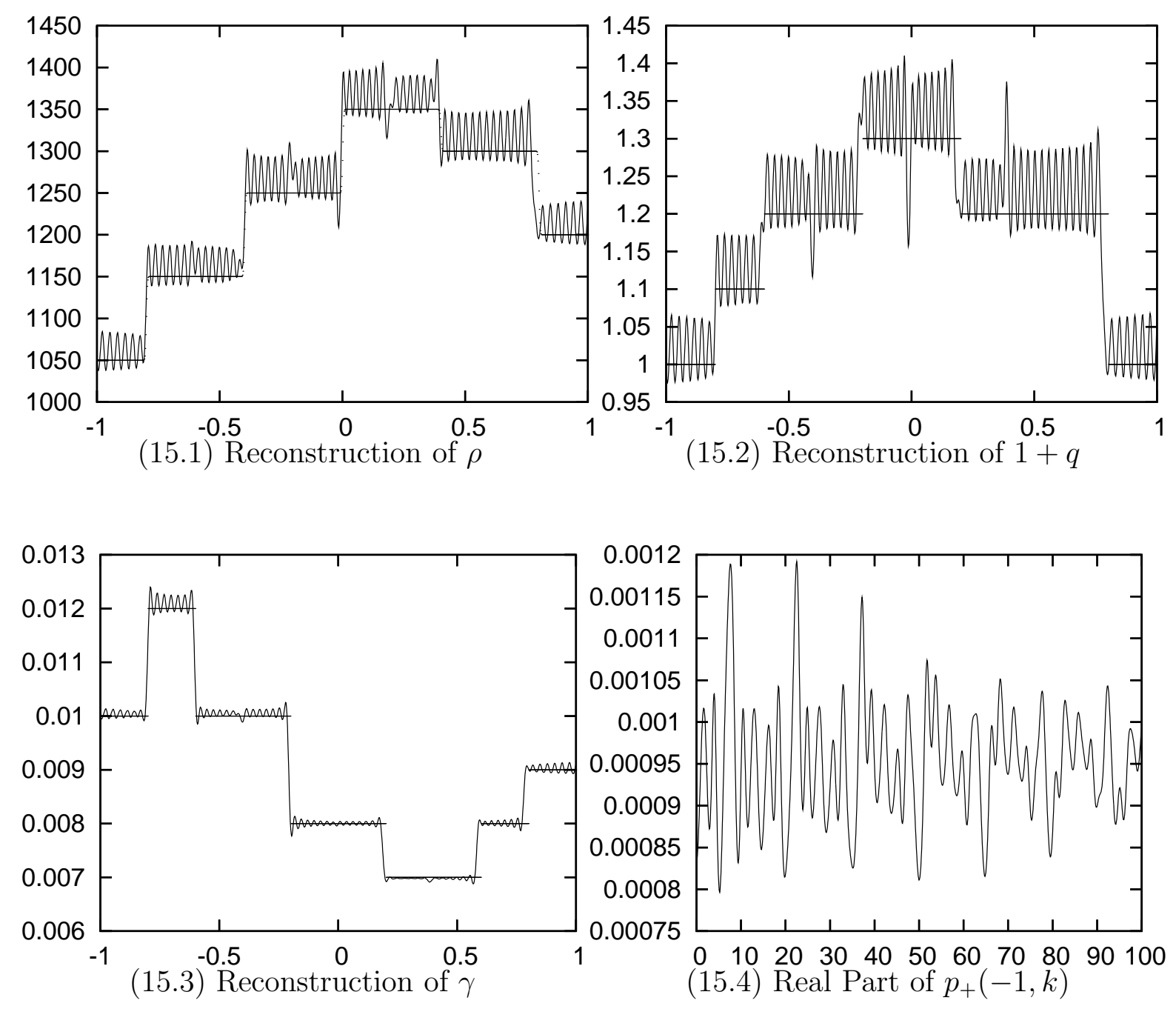

Figure 15: Reconstruction of Example 6 with $a=100$ 

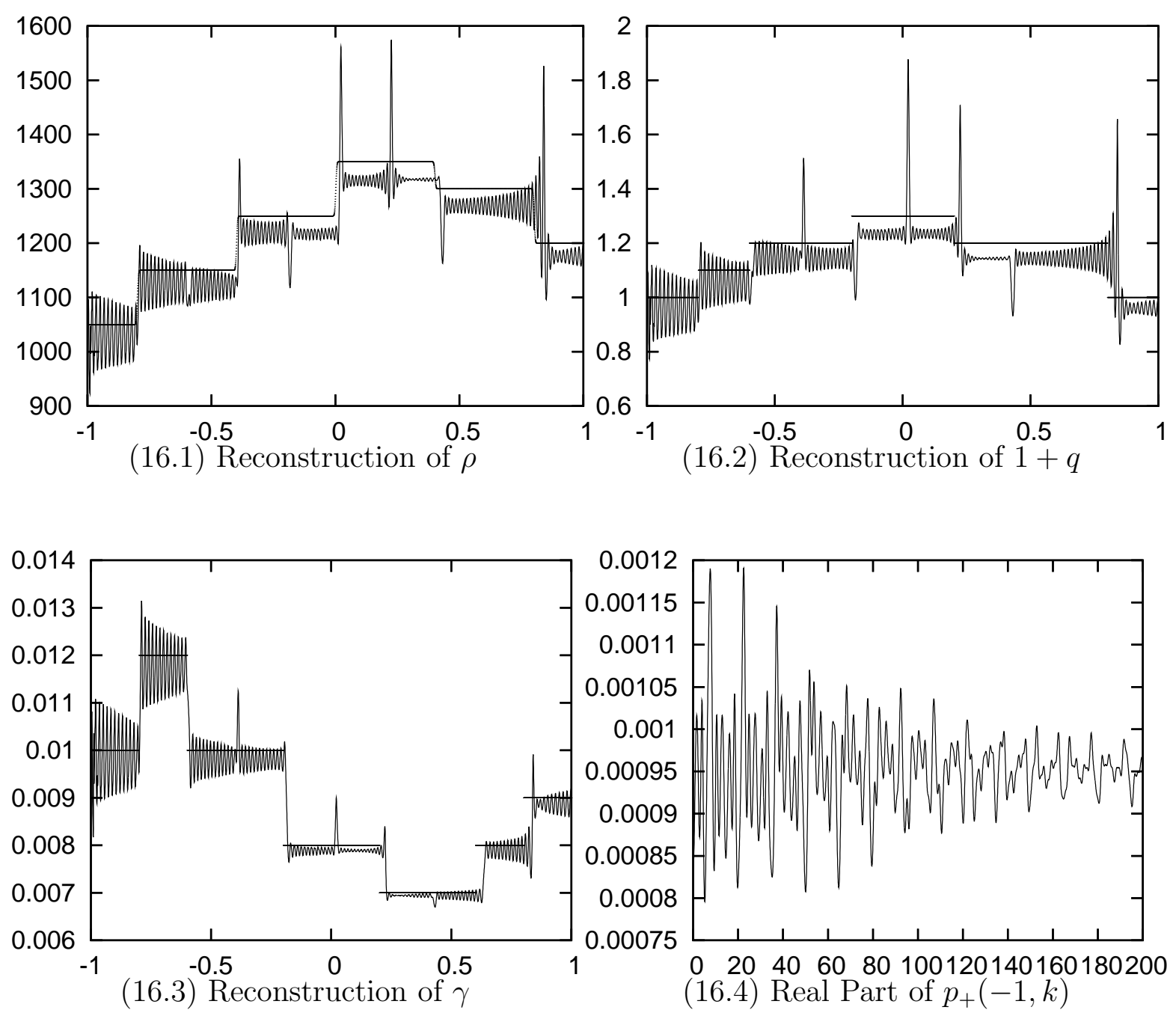

Figure 16: Reconstruction of Example 6 with $a=200$ 

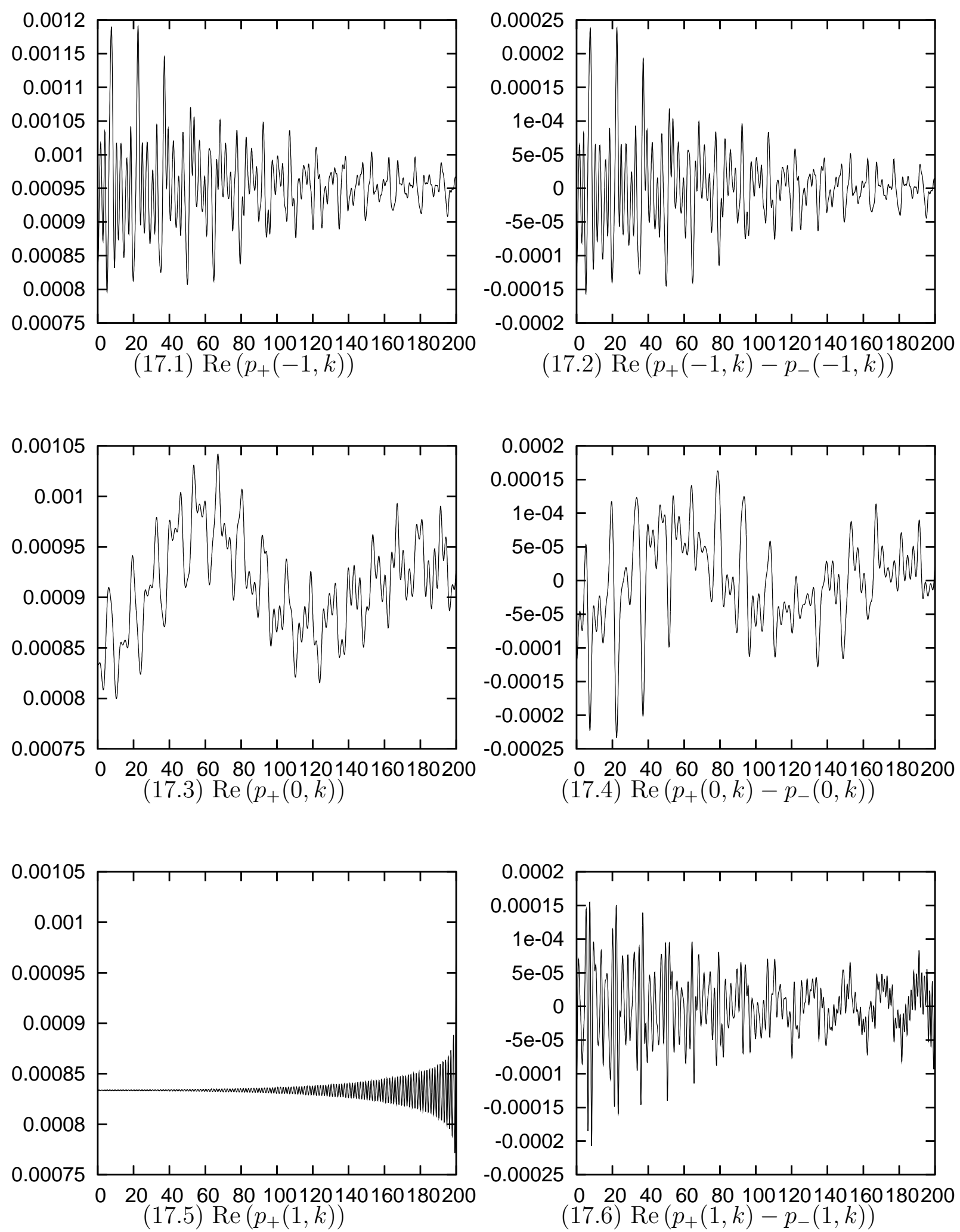

Figure 17: Reconstruction of Example 6 with $a=200$ 
Table 17: CPU time and accuracies for Example 6

\begin{tabular}{|c|c|c|c|c|c|c|}
\hline$a$ & $h_{k}$ & $N_{x}$ & $E_{\rho}^{2}$ & $E_{q}^{2}$ & $E_{\gamma}^{2}$ & $t_{C P U}$ \\
\hline 25 & 0.05 & 500 & $1.81 \mathrm{E}-02$ & $3.77 \mathrm{E}-02$ & $2.83 \mathrm{E}-02$ & $4.2 \mathrm{E}+00$ \\
50 & 0.05 & 1000 & $2.55 \mathrm{E}-02$ & $5.50 \mathrm{E}-02$ & $2.25 \mathrm{E}-02$ & $1.8 \mathrm{E}+01$ \\
100 & 0.1 & 1000 & $2.10 \mathrm{E}-02$ & $4.53 \mathrm{E}-02$ & $2.39 \mathrm{E}-02$ & $1.8 \mathrm{E}+01$ \\
200 & 0.05 & 4000 & $3.77 \mathrm{E}-02$ & $7.85 \mathrm{E}-02$ & $5.06 \mathrm{E}-02$ & $3.5 \mathrm{E}+02$ \\
\hline
\end{tabular}

Table 18: CPU time and accuracies for Example 6

\begin{tabular}{|c|c|c|c|c|c|c|}
\hline$a$ & $h_{k}$ & $N_{x}$ & $E_{\rho}^{\infty}$ & $E_{q}^{\infty}$ & $E_{\gamma}^{\infty}$ & $t_{C P U}$ \\
\hline 25 & 0.05 & 500 & $4.99 \mathrm{E}-02$ & $1.05 \mathrm{E}-01$ & $1.79 \mathrm{E}-01$ & $4.2 \mathrm{E}+00$ \\
50 & 0.05 & 1000 & $5.69 \mathrm{E}-02$ & $1.23 \mathrm{E}-01$ & $1.49 \mathrm{E}-01$ & $1.8 \mathrm{E}+01$ \\
100 & 0.1 & 1000 & $7.22 \mathrm{E}-02$ & $1.78 \mathrm{E}-01$ & $1.95 \mathrm{E}-01$ & $1.8 \mathrm{E}+01$ \\
200 & 0.05 & 4000 & $2.72 \mathrm{E}-01$ & $6.57 \mathrm{E}-01$ & $2.71 \mathrm{E}-01$ & $3.5 \mathrm{E}+02$ \\
\hline
\end{tabular}



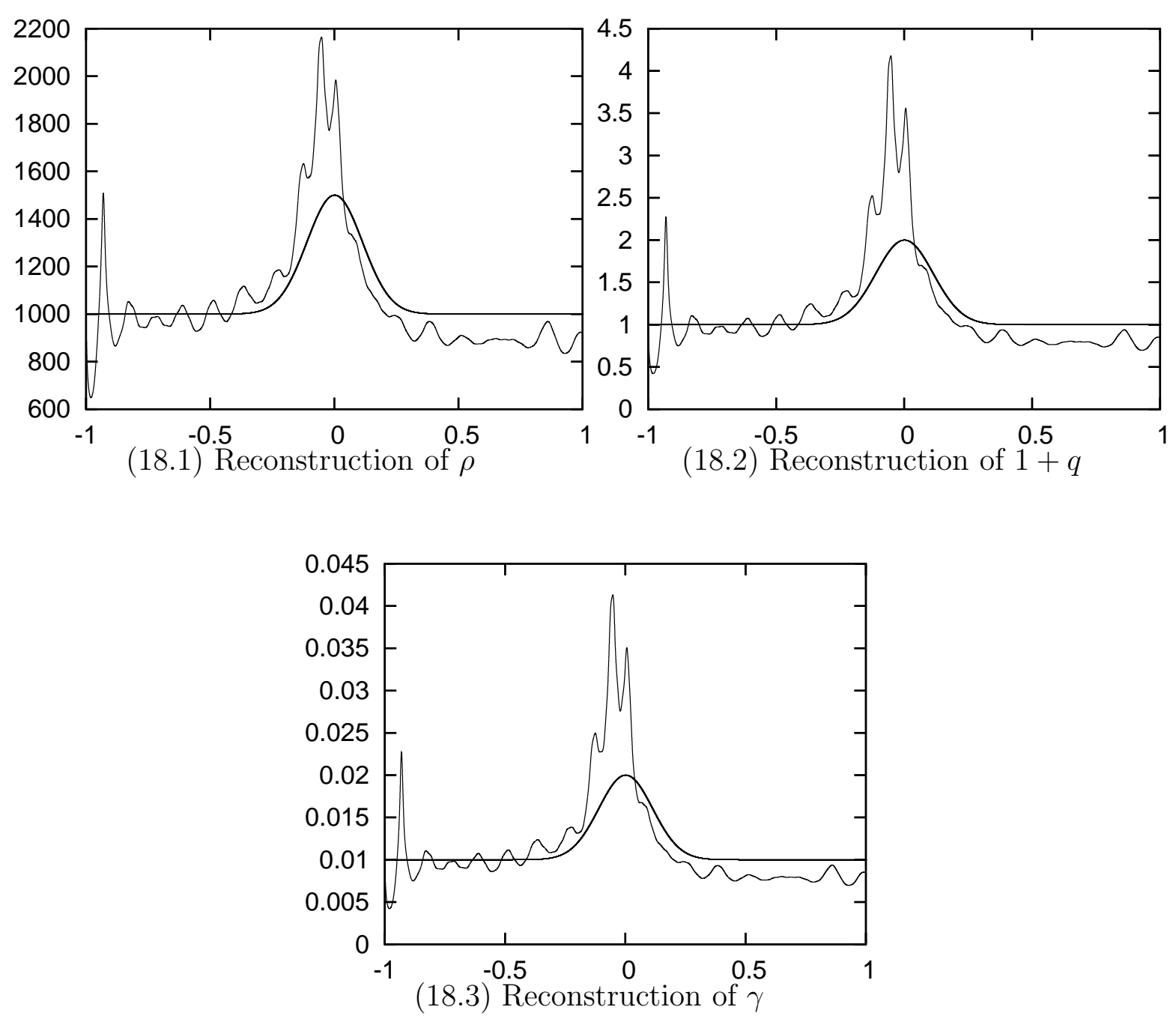

Figure 18: Reconstruction of Example 1 with $\mathrm{D}=3$

Example 7. In this example, we investigate the sensitivity of the reconstruction to perturbations of the initial data. We perturb the initial data for the algorithm by truncating it after a specified number $\mathrm{D}$ of decimal digits (both the real and the imaginary parts). Thus, the maximum relative error is of the order $10^{D-1}$, e.g., when the number 1.999 is truncated after $D=2$ digits, the result is 1.9 .

Tables 19, 20 demonstrates the numerical results of the reconstruction of Example 1 with various truncations of the input data. In each case, $a, h_{k}, N_{x}$ is chosen properly so that the error caused by the inaccuracy of the input data is much larger than that caused by the insufficient $a, h_{k}$ or $N_{x}$. Figures 18, 19, 20, 21 demonstrate the exact and the reconstructed $\rho, q$ and $\gamma$ for different orders of perturbations. 
Table 19: Accuracies for Example 1 with Truncated Data

\begin{tabular}{|c|c|c|c|c|c|c|}
\hline$D$ & $a$ & $h_{k}$ & $N_{x}$ & $E_{\rho}^{2}$ & $E_{q}^{2}$ & $E_{\gamma}^{2}$ \\
\hline 3 & 50 & 0.0125 & 4000 & $1.52 \mathrm{E}-01$ & $3.72 \mathrm{E}-01$ & $3.65 \mathrm{E}-01$ \\
3 & 100 & 0.0125 & 8000 & $1.47 \mathrm{E}-01$ & $3.57 \mathrm{E}-01$ & $3.50 \mathrm{E}-01$ \\
4 & 50 & 0.025 & 2000 & $4.97 \mathrm{E}-02$ & $9.52 \mathrm{E}-02$ & $9.47 \mathrm{E}-02$ \\
4 & 100 & 0.025 & 4000 & $4.99 \mathrm{E}-02$ & $9.53 \mathrm{E}-02$ & $9.48 \mathrm{E}-02$ \\
5 & 50 & 0.025 & 2000 & $1.00 \mathrm{E}-02$ & $2.07 \mathrm{E}-02$ & $2.08 \mathrm{E}-02$ \\
5 & 100 & 0.025 & 4000 & $1.01 \mathrm{E}-02$ & $2.07 \mathrm{E}-02$ & $2.09 \mathrm{E}-02$ \\
6 & 50 & 0.00625 & 8000 & $5.92 \mathrm{E}-04$ & $1.20 \mathrm{E}-03$ & $1.19 \mathrm{E}-03$ \\
6 & 100 & 0.00625 & 16000 & $5.94 \mathrm{E}-04$ & $1.20 \mathrm{E}-03$ & $1.20 \mathrm{E}-03$ \\
\hline
\end{tabular}

Table 20: Accuracies for Example 1 with Truncated Data

\begin{tabular}{|c|c|c|c|c|c|c|}
\hline$D$ & $a$ & $h_{k}$ & $N_{x}$ & $E_{\rho}^{\infty}$ & $E_{q}^{\infty}$ & $E_{\gamma}^{\infty}$ \\
\hline 3 & 50 & 0.0125 & 4000 & $8.22 \mathrm{E}-01$ & $2.32 \mathrm{E}+00$ & $2.31 \mathrm{E}+00$ \\
3 & 100 & 0.0125 & 8000 & $5.09 \mathrm{E}-01$ & $1.27 \mathrm{E}+00$ & $1.27 \mathrm{E}+00$ \\
4 & 50 & 0.025 & 2000 & $1.16 \mathrm{E}-01$ & $2.29 \mathrm{E}+00$ & $2.28 \mathrm{E}+00$ \\
4 & 100 & 0.025 & 4000 & $1.37 \mathrm{E}-01$ & $2.56 \mathrm{E}+00$ & $2.57 \mathrm{E}+00$ \\
5 & 50 & 0.025 & 2000 & $2.02 \mathrm{E}-02$ & $3.97 \mathrm{E}-02$ & $4.00 \mathrm{E}-02$ \\
5 & 100 & 0.025 & 4000 & $2.09 \mathrm{E}-02$ & $4.23 \mathrm{E}-02$ & $4.23 \mathrm{E}-02$ \\
6 & 50 & 0.00625 & 8000 & $1.56 \mathrm{E}-03$ & $2.91 \mathrm{E}-03$ & $2.90 \mathrm{E}-03$ \\
6 & 100 & 0.00625 & 16000 & $1.60 \mathrm{E}-03$ & $3.01 \mathrm{E}-03$ & $2.99 \mathrm{E}-03$ \\
\hline
\end{tabular}



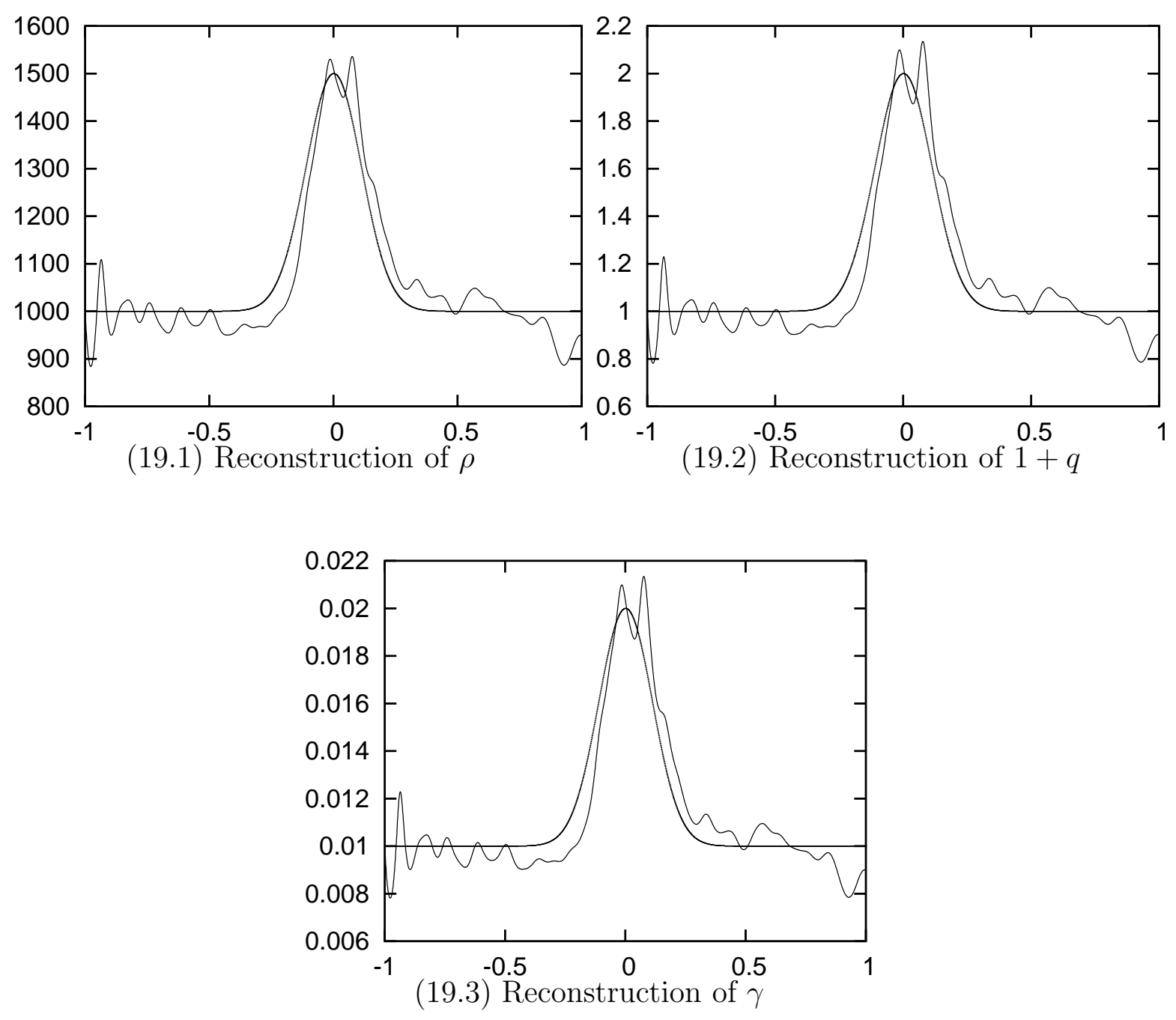

Figure 19: Reconstruction of Example 1 with $\mathrm{D}=4$ 

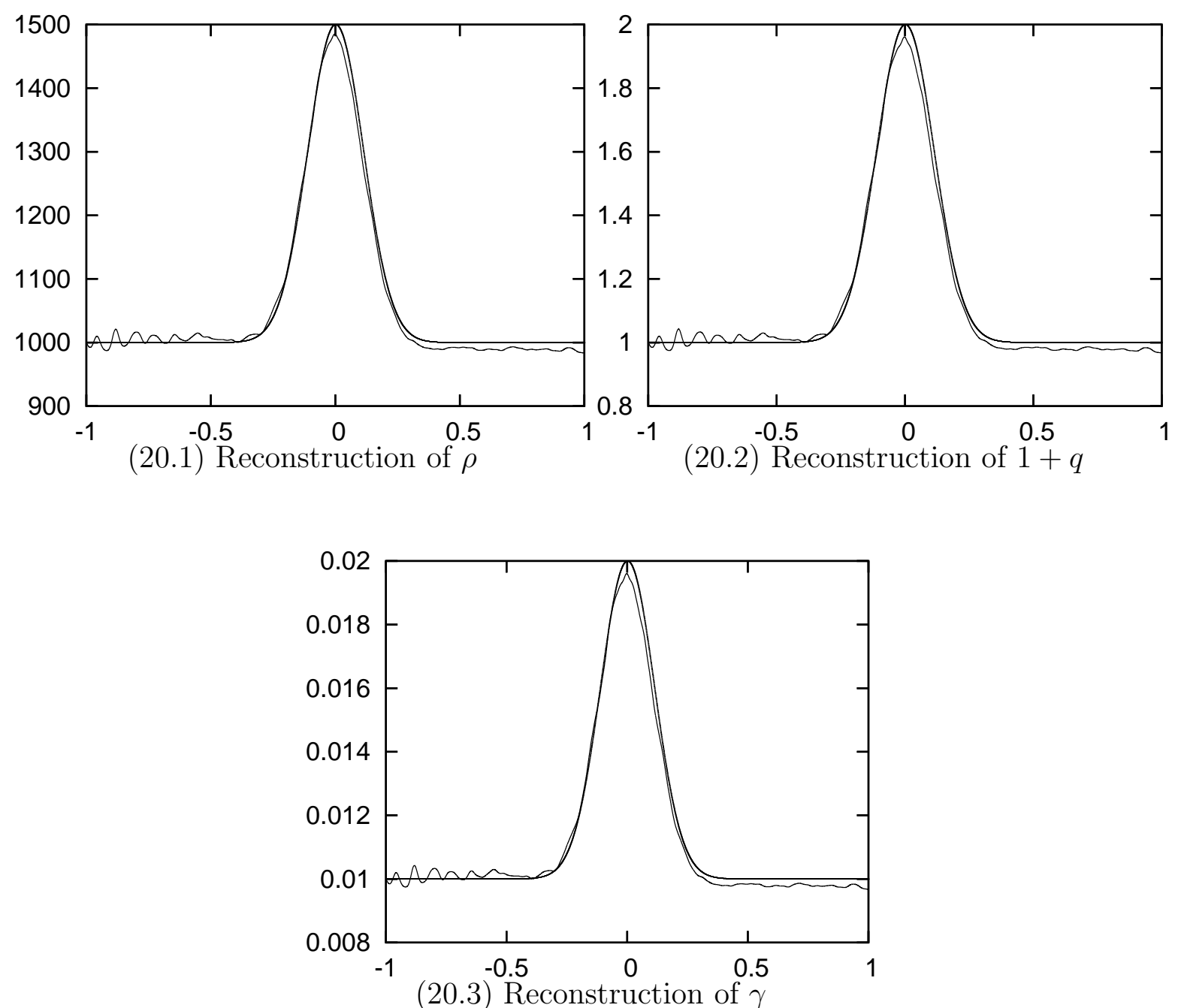

Figure 20: Reconstruction of Example 1 with $\mathrm{D}=5$ 

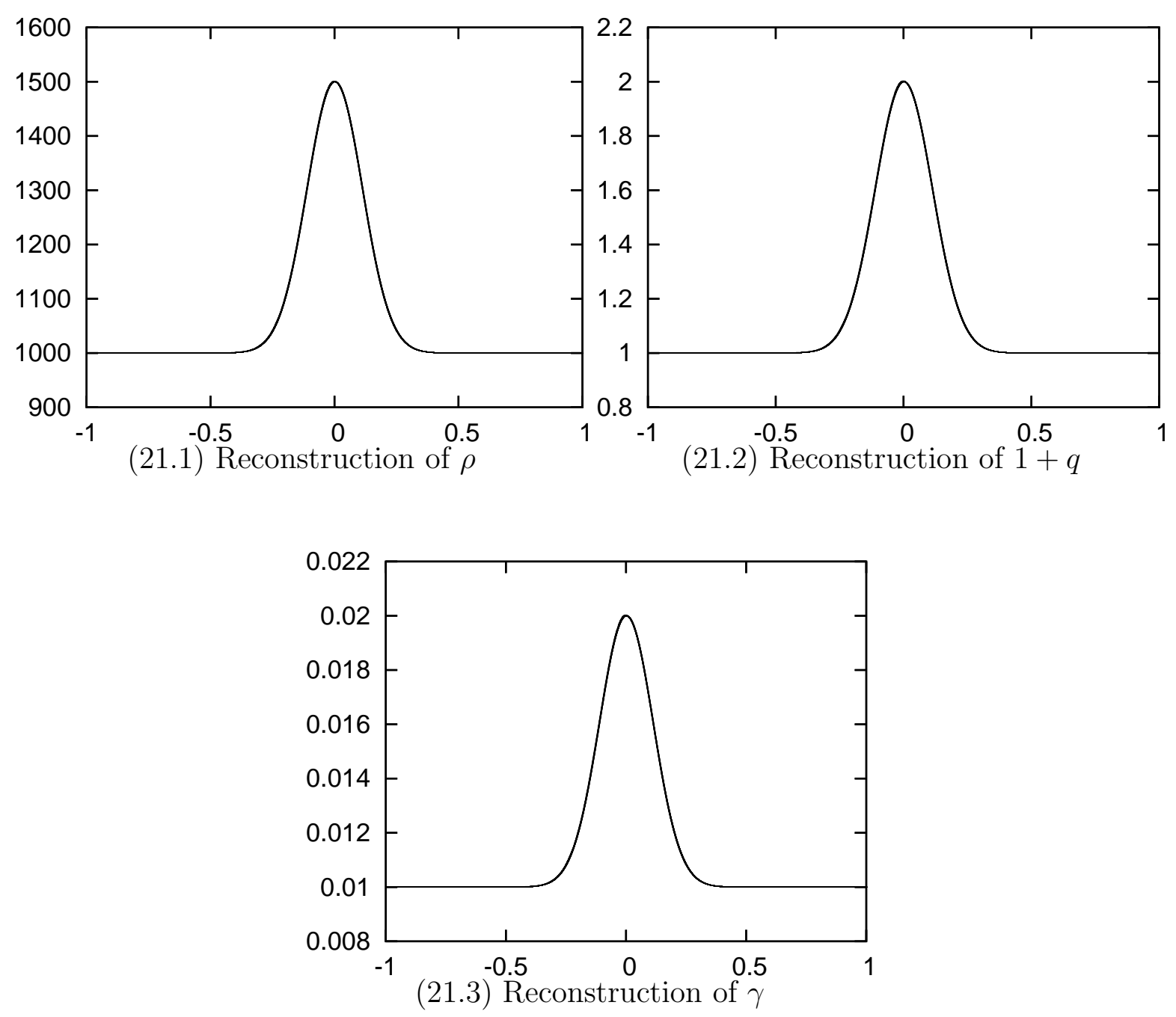

Figure 21: Reconstruction of Example 1 with $D=6$ 
The following observations can be made from the tables above, and from a wide range of numerical tests performed by us.

1. For scatterers satisfying the conditions of Theorem 3.3 (Example 1, 2, 3), the numerical algorithm of Section 4 displays convergence of order $m-1$, where $m$ is the smoothness of the scatterer; the CPU time required is proportional to $N_{k} \cdot N_{x}$, where $N_{k}$ and $N_{x}$ are the numbers of discretization points in frequency and space domain, respectively.

2. For general scatterers violating the conditions of Theorem 3.3 mildly, the algorithm does not converge. However, the algorithm exhibits linear convergence (see Example 4.1, $4.3,4.4)$ for the following two particular categories of scatterers violating the conditions of Theorem 3.3 mildly,

A. $\rho, q$ are continuous but their derivatives are not, and $\gamma$ is absent

B. $q, \gamma$ are continuous but their derivatives are not, and $\rho$ is a constant.

3. When the scatterer is discontinuous (Example 5, 5.1, 6), the algorithm produces results demonstrated in Figures 11 - 17. The oscillatory behavior near the discontinuities is the well-known Gibbs phenomenon. In general, the algorithm is not convergent for such scatterers. However, for scatterers of the following categories

A. $q$ is discontinuous, $\rho$ is a constant, $\gamma$ is absent,

B. $\gamma$ is discontinuous, $\rho$ and $q$ are constants,

the convergence of the algorithm is of the order $O\left(\frac{1}{\sqrt{a}}\right)$, where $a$ is the largest frequency.

4. When the initial data is perturbed (Example 7), the error of the reconstruction is proportional to the magnitude of the perturbation, and the proportion coefficient is 1 .

\section{Conclusions}

In this paper, we construct numerical algorithms for the solution of inverse scattering problems in layered acoustic media in three dimensions. The speed $c$ of propagation of sound, the density $\rho$, and the attenuation $\gamma$ are the three parameters reconstructed by the algorithm. The computational complexity of the algorithm is $O\left(N_{k} \cdot N_{x}\right)$, where $N_{k}, N_{x}$ are the number of discretization points in the frequency and space domains, respectively.

The inverse scattering schemes we construct can be viewed as an extension of [3], and are based on a collection of trace formulae, which connect the Fourier components of the solutions of the Helmholtz equation to the parameters being recovered. Under the assumption of mild attenuation and a smoothly varying medium, our inverse scattering algorithms require only a few measurements, in the sense that given a medium whose parameters $c, \rho$, and $\gamma$ have $m \geq 1$ continuous derivatives, and data measured for all frequencies $\omega$ in the interval $[-a, a]$, the error of the reconstruction decays as $1 / a^{m-1}$ as $a \rightarrow \infty$. In this respect, our algorithm is similar to the Fourier transform.

Acknowledgments. The authors would like to thank M. Tygert for constructive discussions. 


\section{References}

[1] M. Abramowitz and I. Stegun, Handbook of Mathematical Functions, Applied Math. Series (National Bureau of Standards), Washington, DC, 1964.

[2] Y. Chen, Inverse Scattering via Heisenberg's Uncertainty Principle, Inverse Problems. Vol. 13, No.2, pp. 253-282, 1997.

[3] Y. Chen, V. Rokhlin, On the Inverse Scattering Problem for the Helmholtz Equation in One Dimension, Inverse Problems. Vol. 8, pp. 365-391, 1992.

[4] E. A. Coddington, N. Levinson Theory of Ordinary Differential Equations, McGraw Hill, New York, 1955.

[5] D. L. Colton, M. Piana, R. Potthast, A Simple Method Using Morozov's Discrepancy Principle for Solving Inverse Scattering Problems, Inverse Problems. Vol. 13, pp. 14771493, 1997.

[6] R. Duan, V. Rokhlin, On the inverse scattering problem in the acoustic environment, Technical Report, YALEU/DCS/TR-1395, 2008.

[7] I. W. Gel'fand, B. M. Levitan, On the determination of a differential equation by its spectra function, Dokl. Akad. Nauk. USSR Vol. 77, pp. 557-560, 1951.

[8] A. Glaser, V. Rokhlin, A new class of highly accurate solvers for ordinary differntial equations, Technical Report, YALEU/DCS/TR-1382, 2007.

[9] M. Kaveh, M. Soumekh, Computer-assisted Diffraction Tomography, Image Recovery: Theory and Application. pp. 369-413, 1987.

[10] J. H. Lin, W. C. Chew, Solution of the three-dimensional electromagnetic inverse problem by the local shape function and the conjugate gradient fast Fourier transform methods, Journal of the Optical Society of America. Vol. 14, pp. 3037-3045, 1997.

[11] D. C. Stickler, Application of the Trace Formula Methods to Inverse Scattering for Some Geophysical Problems, Inverse Problems. Vol. 44, pp. 1218-1225, 1984.

[12] J. Stoer, R. Bulirsch Introduction to Numerical Analysis, Springer, Berlin, 1984.

[13] W. W. Symes, J. J. Carazzone, Velocity Inversion by Differential Semblance Optimization, Geophysics, Vol. 56, pp. 654-663, 1991. 